\title{
SINGULAR HOLOMORPHIC FOLIATIONS BY CURVES I: INTEGRABILITY OF HOLONOMY COCYCLE IN DIMENSION 2
}

\author{
VIÊT-ANH NGUYÊN \\ Dedicated to Professor Nessim Sibony for his seventieth birthday
}

\begin{abstract}
We study the holonomy cocycle $\mathcal{H}$ of a holomorphic foliation $\mathscr{F}$ by Riemann surfaces defined on a compact complex projective surface $X$ satisfying the following two conditions:

- its singularities $E$ are all hyperbolic;

- there is no holomorphic non-constant map $\mathbb{C} \rightarrow X$ such that out of $E$ the image of $\mathbb{C}$ is locally contained in a leaf.

Let $T$ be a harmonic current tangent to $\mathscr{F}$ which does not give mass to any invariant analytic curve. Using the leafwise Poincaré metric, we show that $\mathcal{H}$ is integrable with respect to $T$. Consequently, we infer the existence of the Lyapunov exponent function of $T$.
\end{abstract}

Classification AMS 2010: Primary: 37F75, 37A30; Secondary: 57R30, 58J35, 58J65, 60J65.

Keywords: holomorphic foliation, hyperbolic singularity, Poincaré metric, harmonic current, holonomy cocycle, Lyapunov exponent.

\section{CONTENTS}

1. Introduction

1.1. General settings and main results

1.2. Outline of the proofs

1.3. Organization of the article and acknowledgments

2. Background

2.1. Foliations, singularities, Poincaré metric and Brody hyperbolicity

2.2. A local model

2.3. Heat diffusions and harmonic currents versus harmonic measures

2.4. Measure theory on sample-path spaces

2.5. Holonomy cocycles

3. Holonomy cocycle vs Poincaré metric

4. Proof of the main results modulo the integrability condition

5. Harmonic currents on the local model

6. Proof of the integrability condition: First reduction

7. Geometric intersection and interpretations 
8. Test curves $\mathfrak{C}_{r}, \mathfrak{C}_{r, N} \ldots$ and second reduction 37

9. Cohomological relation and third reduction 42

10. Intersection of test curves with a leaf 44

11. Mass of $T \wedge\left[\mathfrak{C}_{r}\right]$ on balls

12. Mass of $T \wedge\left[\mathfrak{C}_{r, N}\right]$ outside the corona $\mathbb{A}_{r, N} \quad 59$

13. Mass of $T \wedge\left[\mathfrak{C}_{r}\right], T \wedge\left[\mathfrak{C}_{0, N}\right], T \wedge\left[\mathfrak{C}_{r, N}\right]$ on the corona $\mathbb{A}_{r, N}$

13.1. Mass of $T \wedge\left[\mathfrak{C}_{r}\right]$ on $\mathbb{A}_{r, N} \quad 64$

13.2. Mass of $T \wedge\left[\mathfrak{C}_{0, N}\right]$ on $\mathbb{A}_{r, N} \quad 66$

13.3. Mass of $T \wedge\left[\mathfrak{C}_{r, N}\right]$ on $\mathbb{A}_{r, N} \quad 68$

14. Completion of the reductions $\quad 70$

14.1. End of the proof of Proposition $8.6 \quad 71$

\begin{tabular}{ll|} 
14.2. End of the proof of Proposition 8.4 & 73 \\
\hline
\end{tabular}

\begin{tabular}{ll} 
References & 73 \\
\hline
\end{tabular}

\section{INTRODUCTION}

1.1. General settings and main results. The dynamical and geometric theory of holomorphic foliations by curves has received much attention in the past few years. The holonomy cocycle (or equivalently, the normal derivative cocycle) of a foliation is a very important object which reflects dynamical as well as geometric and analytic aspects of the foliation. Exploring this object allows us to understand more about the foliation itself. Let $\mathscr{F}=(X, \mathscr{L}, E)$ be a holomorphic foliation by hyperbolic Riemann surfaces which is immersed onto an ambient complex manifold $X$ and which possesses the set of singularities $E$. On the geometric side, we have harmonic currents $T$ which are generalizations of the foliations cycles introduced by Sullivan [29]. On the dynamical side, the sample-path space $\Omega$ associated to the foliation describes the leafwise Brownian motion with respect to the Poincaré metric on leaves. This motion generates a Markov process on $X$.

Assume for the moment that $\mathscr{F}$ does not possess any singularities (i.e. $E=$ $\varnothing)$. Let $T$ be a harmonic current tangent to $\mathscr{F}$. When $X$ is a surface, i.e. $\operatorname{dim} X=2$, we can define the unique Lyapunov exponent function of $T$, which is leafwise constant and which measures heuristically the exponential rate of convergence of leaves toward each other along leafwise Brownian trajectories (see Candel [3], Deroin [8]). When $\operatorname{dim} X \geq 2$, our recent work in [25] provides $(\operatorname{dim} X-1)$ Lyapunov exponent functions whose geometric characterizations in terms of geodesic rays have been investigated in [26].

Since the main examples of holomorphic foliations by curves are those in the complex projective space $\mathbb{P}^{k}$ of arbitrary dimension (in which case there are always singularities) or in algebraic manifolds, the following fundamental question arises naturally: 
Question. Can one define the Lyapunov exponent functions of a harmonic current $T$ tangent to a singular holomorphic hyperbolic foliation $\mathscr{F}=(X, \mathscr{L}, E)$ ?

The main purpose of this paper is to give an affirmative answer to this question for generic foliations, that is, when the ambient manifold $X$ is a compact complex projective surface, the foliation enjoys Brody hyperbolicity (see Definition 2.1 below), and $E$ is the set of singularities which are of hyperbolic type.

Here is our main result. The new terminology and notation appearing in this theorem will be explained in Section 2 below.

Theorem 1.1. Let $\mathscr{F}=(X, \mathscr{L}, E)$ be a holomorphic Brody hyperbolic foliation with hyperbolic singularities $E$ in a Hermitian compact complex projective surface $X$. Let $\mathcal{H}$ be the holonomy cocycle of the foliation. Let $T$ be a harmonic current tangent to $\mathscr{F}$ which does not give mass to any invariant analytic curve. Consider the corresponding harmonic measure $\mu:=T \wedge g_{P}$ where $g_{P}$ is the leafwise Poincaré metric. Let $\Omega$ be the sample-path space associated with $\mathscr{F}$. Let $\bar{\mu}$ be the invariant measure on $\Omega$ associated with $\mu$. Consider the function $\mathscr{I}: \Omega \rightarrow \mathbb{R}^{+}$defined by

$$
\mathscr{I}(\omega):=\sup _{t \in[0,1]}|\log \|\mathcal{H}(\omega, t)\||, \quad \omega \in \Omega .
$$

Then $\mathscr{I}$ is $\bar{\mu}$-integrable.

Here is an immediate consequence of this theorem.

Corollary 1.2. Under the hypotheses and notation of Theorem 1.1. assume in addition that the measure $\mu$ is ergodic. Then $T$ admits the (unique) Lyapunov exponent $\lambda(T)$ given by the formula

$$
\lambda(T):=\int_{\Omega} \log \|\mathcal{H}(\omega, 1)\| d \bar{\mu}(\omega) .
$$

Moreover, for $\mu$-almost every $x \in X$, we have

$$
\lim _{t \rightarrow \infty} \frac{1}{t} \log \|\mathcal{H}(\omega, t)\|=\lambda(T)
$$

for almost every path $\omega \in \Omega$ with respect to the Wiener measure at $x$ which lives on the leaf passing through $x$.

For comprehensive expositions on characterization of Lyapunov exponents using the Wiener measures, see [3, 9, 25, 26]. In Theorem 2.7 below, we will see that the measure $\mu$ is ergodic when, for example, the current $T$ is an extremal point in the convex cone of all harmonic currents tangent to $\mathscr{F}$.

Consider a singular foliation by curves $\mathscr{F}=\left(\mathbb{P}^{2}, \mathscr{L}, E\right)$ on the complex projective plane $\mathbb{P}^{2}$ such that all the singularities of $\mathscr{F}$ are hyperbolic and that $\mathscr{F}$ has no invariant algebraic curve. Combining some results by Glutsyuk [19] and by Lins Neto [23], and by Brunella [2], we will see in Remark 2.2 and in the discussion after Theorem 2.3 below that $\mathscr{F}$ is Brody hyperbolic. Moreover, the 
unique ergodicity theorem of Fornæss-Sibony [16] says that the harmonic current $T$ is unique up to a multiplicative constant. In particular, the convex cone of all harmonic currents of $\mathscr{F}$ is just a real half-line, and hence all these currents are extremal (see the discussion preceding Theorem 2.7 below). Therefore, the measure $T \wedge g_{P}$ is ergodic by Part 2) of this theorem. Consequently, Corollary 1.2 applies and gives us the following result. It can be applied to every generic foliation in $\mathbb{P}^{2}$ with a given degree $d>1$.

Corollary 1.3. Let $\mathscr{F}=\left(\mathbb{P}^{2}, \mathscr{L}, E\right)$ be a singular foliation by curves on the complex projective plane $\mathbb{P}^{2}$. Assume that all the singularities are hyperbolic and that $\mathscr{F}$ has no invariant algebraic curve. Let $T$ be the unique harmonic current tangent to $\mathscr{F}$ such that $\mu:=T \wedge g_{P}$ is a probability measure. Let $\mathcal{H}, \bar{\mu}$ and $\mathscr{I}$ be as in the statement of Theorem 1.1. Then the conclusion of this theorem as well as that of Corollary 1.2 hold. In particular, $\mathscr{F}$ admits a unique Lyapunov exponent.

The novelty of the last corollary is that the (unique) Lyapunov exponent of such a foliation $\mathscr{F}$ is intrinsic and canonical.

In fact, we will prove the following more complete version of Theorem 1.1 where we introduce the so-called integrability condition.

Theorem 1.4. Let $\mathscr{F}=(X, \mathscr{L}, E)$ be a holomorphic Brody hyperbolic foliation with hyperbolic singularities $E$ in a compact complex projective surface $X$. Let $T$ be a harmonic current tangent to $\mathscr{F}$ which does not give mass to any invariant analytic curve. Then we have

$$
\text { (1.1) (the integrability condition): } \quad \int_{X}|\log \operatorname{dist}(x, E)| \cdot\left(T \wedge g_{P}\right)(x)<\infty \text {. }
$$

Using the Poincaré metric of the punctured disc as a local model and Lemma 2.4 below, we can prove that if the harmonic current $T$ has a positive mass on an invariant analytic curve, then the integral in (1.1) is infinite, i.e., the integrability condition breaks down.

The condition of Brody hyperbolicity seems to be indispensable for the integrability of the holonomy cocycle. Indeed, in a very recent work [21, Theorem A] Hussenot discovers the following remarkable property for a class of Ricatti foliations $\mathscr{F}$ on $\mathbb{P}^{2}$. For every $x \in \mathbb{P}^{2}$ outside invariant curves of every foliation in this class, it holds that

$$
\limsup _{t \rightarrow \infty} \frac{1}{t} \log \|\mathcal{H}(\omega, t)\|=\infty
$$

for almost every path $\omega \in \Omega_{x}$ with respect to the Wiener measure at $x$ which lives on the leaf passing through $x$. By Glutsyuk [19] and Lins Neto [22], these foliations are hyperbolic since all their singular points have nondegenerate linear part. Nevertheless, neither of them is Brody hyperbolic because they all contain integral curves which are some images of $\mathbb{P}^{1}$ (see Remark 2.2 below). 
1.2. Outline of the proofs. Now we discuss the method of the proof of Theorems 1.1 and 1.4. Our approach consists of two main steps.

In the first main step we show that Theorem 1.1 follows from Theorem 1.4 , i.e., from the integrability condition (1.1). To this end we study the behavior of the holonomy cocycle near the singularities with respect to the leafwise Poincaré metric. Let $g_{X}$ be a Hermitian metric on $X$ and let dist denote the distance on $X$ induced by $g_{X}$. Roughly speaking, this step quantifies the expansion speed of the hololomy cocycle in terms of the ambient metric $g_{X}$ when one travels along unit-speed geodesic rays. The main ingredients are in our joint-works with Dinh and Sibony in [10, 11, 12].

The second main step is then devoted to the proof of Theorem 1.4, i.e., inequality (1.1). The main difficulty is that known estimates (see, for example, [10]) on the behavior of $T$ near linearizable singularities, only give a weaker inequality

$$
\int_{X}|\log \operatorname{dist}(x, E)|^{1-\delta} \cdot\left(T \wedge g_{P}\right)(x)<\infty, \quad \forall \delta>0 .
$$

So (1.1) is the limiting case of (1.2). The proof of (1.2) in [10, Proposition 4.2] relies on the finiteness of the Lelong number of $T$ at every point. Recall from Skoda [28] that the Lelong number of a harmonic current at a given point is an important indicator measuring the mass-density of the current at that point. Moreover, our result in [27] (see also a recent result of Dinh-Sibony [13]) sharpens the last estimate by showing that the Lelong number of $T$ vanishes at every hyperbolic singular point $x \in E$. Nevertheless, even this better estimate does not suffice to prove (1.1).

To overcome this obstacle, we use a cohomological idea which exploits fully the assumption that $X$ is projective. This assumption imposes a stronger massclustering condition on harmonic currents.

Now we explain briefly our proof of the integrability condition (1.1). Our approach is based on a cohomological invariance (see Proposition 9.3) which says roughly that if two algebraic curves $\mathfrak{C}$ and $\mathfrak{D}$ on $X$ are cohomologous (for example, if they have the same algebraic degree when $X=\mathbb{P}^{2}$ ), then under suitable assumptions, we can define the wedge-product $T \wedge[\mathfrak{C}], T \wedge[\mathfrak{D}]$ which are finite positive Borel measures and their masses are equal, i.e,

$$
\int_{X} T \wedge[\mathfrak{C}]=\int_{X} T \wedge[\mathfrak{D}] .
$$

Before going further, let us explain why equality (1.3) could be true. Since $\mathfrak{C}$ and $\mathfrak{D}$ on $X$ are cohomologous on $X$, the $\partial \bar{\partial}$-lemma for compact Kähler manifolds provides us an integrable function $u$ on $X$ such that

$$
[\mathfrak{C}]-[\mathfrak{D}]=i \partial \bar{\partial} u \quad \text { in the sense of currents. }
$$


So we can write

$$
\int_{X} T \wedge[\mathfrak{C}]-\int_{X} T \wedge[\mathfrak{D}]=\int_{X} T \wedge i \partial \bar{\partial} u
$$

The function $u$ is, in general, not smooth near $\mathfrak{C}$ and $\mathfrak{D}$. However, if we could consider it like a smooth function, Stokes' theorem would turn the right hand side of the last line into the following integral

$$
\int_{X} u(i \partial \bar{\partial} T)=0
$$

where the last equality holds since the harmonicity of $T$ implies that $i \partial \bar{\partial} T=0$. Therefore, we may expect equality (1.3) to hold.

Resuming the sketchy proof of the integrability condition (1.1), let $x_{0} \in E$ and fix a coordinate system $(z, w)$ around $x_{0}$ such that the two separatrices of the hyperbolic singular point $x_{0}$ are $\{z=0\}$ and $\{w=0\}$. Then we can show that the vanishing of the Lelong number of $T$ at 0 established in [27] is equivalent to the following convergence

$$
\int_{\mathbb{B}(0, r)} T \wedge[z=r] \rightarrow 0 \quad \text { as } \quad r \rightarrow 0,
$$

where $\mathbb{B}(0, r)$ is the ball in $X$ with center $x_{0}=0$ and radius $r$. And more importantly, the integrability condition (1.1) is somehow equivalent to the statement that the convergence (1.4) has, in a certain very weak sense, a speed of order $|\log r|^{-\delta}$ as $r \rightarrow 0$ for some $\delta>0$. Note, however, that this speed does not at all mean that $\int_{\mathbb{B}(0, r)} T \wedge[z=r]=O\left(|\log r|^{-\delta}\right)$. For a precise meaning of this speed, see Remark 6.3 below.

Now suppose for the sake of simplicity that $X=\mathbb{P}^{2}$ and $N \in \mathbb{N}$ is large enough. We choose an algebraic curve $\mathfrak{C}$ of degree $N$ which looks like the analytic curve $\left\{z=w^{N}\right\}$ near 0 . We also choose an algebraic curve $\mathfrak{D}$ of degree $N$ which looks like the analytic curve $\left\{r=z-w^{N}\right\}$ near 0 . The following seven observations play a key role in our approach, where $0<\delta<1$ is an exponent independent of $r$ and $N, 0<r<r_{0}$ with $r_{0}>0$ a fixed small number.

(i) Outside a small ball $\mathbb{B}\left(0, r_{0}\right)$, the analytic curve $\left\{z=w^{N}\right\}$ (and hence the algebraic curve $\mathfrak{C}$ ) falls into a tubular neighborhood with size $O\left(r^{\rho}\right)$ of the analytic curve $\left\{r=z-w^{N}\right\}$ (and hence the algebraic curve $\mathfrak{D}$ ), where $\rho$ is a real number depending on $N$ with $0<\rho \leq 1$. So we may expect

$$
\int_{X \backslash \mathbb{B}\left(0, r_{0}\right)} T \wedge[\mathfrak{C}]=\int_{X \backslash \mathbb{B}\left(0, r_{0}\right)} T \wedge[\mathfrak{D}]+O\left(r^{\rho}\right) .
$$

(ii) Outside the ball $\mathbb{B}\left(0, r^{1 / N}|\log r|^{3 / N}\right)$ and inside the small ball $\mathbb{B}\left(0, r_{0}\right)$, the analytic curve $\left\{r=z-w^{N}\right\}$ (and hence the algebraic curve $\mathfrak{D}$ ) behaves like the analytic curve $\left\{z=w^{N}\right\}$ (and hence the algebraic curve $\mathfrak{C}$ ) while intersecting 
the two curves with a general leaf. Indeed, when $|w| \geq r^{1 / N}|\log r|^{3 / N}$, we have $r \ll|w|^{N}$. So we may expect

$$
\int_{\mathbb{B}\left(0, r_{0}\right) \backslash \mathbb{B}\left(0, r^{1 / N}|\log r|^{3 / N}\right)} T \wedge[\mathfrak{C}]=\int_{\mathbb{B}\left(0, r_{0}\right) \backslash \mathbb{B}\left(0, r^{1 / N}|\log r|^{3 / N}\right)} T \wedge[\mathfrak{D}]+O\left(|\log r|^{-\delta}\right) .
$$

(iii) The corona $\mathbb{A}_{r, N}:=\mathbb{B}\left(0, r^{1 / N}|\log r|^{3 / N}\right) \backslash \mathbb{B}\left(0, r^{1 / N}|\log r|^{-3 / N}\right)$ is, in some sense, small and it may be considered as negligible. So we may expect

$$
\int_{\mathbb{A}_{r, N}} T \wedge[\mathfrak{C}]=O\left((\log r)^{-\delta}\right) \quad \text { and } \quad \int_{\mathbb{A}_{r, N}} T \wedge[\mathfrak{D}]=O\left(|\log r|^{-\delta}\right) .
$$

(iv) Our next observation is the following partition of $X$ for $0<r \ll 1$ :

$$
X=\left(X \backslash \mathbb{B}\left(0, r_{0}\right)\right) \coprod\left(\mathbb{B}\left(0, r_{0}\right) \backslash \mathbb{B}\left(0, r^{1 / N}|\log r|^{3 / N}\right)\right) \coprod \mathbb{A}_{r, N} \coprod \mathbb{B}\left(0, r^{1 / N}|\log r|^{-3 / N}\right) .
$$

This allows us to decompose both integrals of (1.3) into corresponding pieces.

Consequently, when the degree $N$ is sufficiently high, by taking into account the observations $(i)-(i i)-(i i i)-(i v)$, and using (1.3), we see that

$$
\int_{\mathbb{B}\left(0, r^{1 / N}|\log r|^{-3 / N}\right)} T \wedge[\mathfrak{C}]-\int_{\mathbb{B}\left(0, r^{1 / N}|\log r|^{-3 / N}\right)} T \wedge[\mathfrak{D}]=O\left(|\log r|^{-\delta}\right) .
$$

(v) Inside the ball $\mathbb{B}\left(0, r^{1 / N}|\log r|^{-3 / N}\right)$, the analytic curve $\left\{z=w^{N}\right\}$ (and hence the algebraic curve $\mathfrak{C}$ ) clusters around 0 , in a certain sense, much more often than the analytic curve $\{z=r\}$ (and hence the algebraic curve $\mathfrak{D}$ ). Indeed, we see in the equation $z=w^{N}$ that both $z$ and $w$ can tend to 0 , whereas in the equation $z=r$, only $w$ could tend to 0 . So we may expect that in a certain sense,

$$
\int_{\mathbb{B}\left(0, r^{1 / N}|\log r|^{-3 / N}\right)} T \wedge[\mathfrak{D}] \ll \int_{\mathbb{B}\left(0, r^{1 / N}|\log r|^{-3 / N}\right)} T \wedge[\mathfrak{C}]
$$

This, combined with the estimate obtained just before $(v)$, implies that both integrals

$$
\int_{\mathbb{B}\left(0, r^{1 / N}|\log r|^{-3 / N}\right)} T \wedge[\mathfrak{C}] \text { and } \int_{\mathbb{B}\left(0, r^{1 / N}|\log r|^{-3 / N}\right)} T \wedge[\mathfrak{D}]
$$

admit, in a certain sense, a speed of order $|\log r|^{-\delta}$.

(vi) Inside the ball $\mathbb{B}\left(0, r^{1 / N}|\log r|^{-3 / N}\right)$, the analytic curve $\left\{r=z-w^{N}\right\}$ (and hence the algebraic curve $\mathfrak{D}$ ) behaves like the analytic curve $\{z=r\}$ while intersecting the two curves with a general leaf. Indeed, when $|w| \leq$ $r^{1 / N}|\log r|^{-3 / N}$, we have $|w|^{N} \ll r$. So we may expect

$$
\int_{\mathbb{B}\left(0, r^{1 / N}|\log r|^{-3 / N}\right)} T \wedge[\mathfrak{D}]-\int_{\mathbb{B}\left(0, r^{1 / N}|\log r|^{-3 / N}\right)} T \wedge[z=r]=O\left(|\log r|^{-\delta}\right) .
$$

This, together with the estimate just obtained before $(v i)$, yields that

$$
\int_{\mathbb{B}\left(0, r^{1 / N}|\log r|^{-3 / N}\right)} T \wedge[z=r]
$$


has, in a certain sense, a speed of order $|\log r|^{-\delta}$.

(vii) Our last observation is that one can show that there is a constant $c_{N}>1$ independent of $r$ such that

$$
c_{N}^{-1} \int_{\mathbb{B}\left(0, r^{1 / N}\right)} T \wedge[z=r] \leq \int_{\mathbb{B}(0, r)} T \wedge[z=r] \leq c_{N} \int_{\mathbb{B}\left(0, r^{1 / N}\right)} T \wedge[z=r] .
$$

This, together with the estimate just obtained before (vii), implies that

$$
\int_{\mathbb{B}(0, r)} T \wedge[z=r]
$$

admits, in a certain sense, a speed of order $|\log r|^{-\delta}$. Hence, we get the convergence with speed of (1.4). This is what we are looking for.

In fact, the factor $|\log r|^{3 / N}$ appearing in the above observations comes from the degeneration of the Poincaré metric $g_{P}$ relative to the ambient metric $g_{X}$ (see formula (2.1D). Moreover, the larger the degree $N$ is, the more evident the mass-clustering phenomenon in the previous observation becomes.

Our approach underlines several tasks. On the one hand, we need to define a geometric intersection of a harmonic current with a singular analytic curve defined on a neighborhood of a singular point of the foliation. On the other hand, we need to approximate some (local) analytic curves by global algebraic ones. The assumption of projectivity of $X$ is needed in order to ensure a good supply of algebraic curves.

1.3. Organization of the article and acknowledgments. The article is organized as follows.

In Section 2 below we set up the background and prepare the auxiliary results. Some basic facts extracted from [10, 11, 12] about the behavior of the leafwise Poincaré metric near the singularities are recalled here. A quick discussion on the heat diffusions as well as the measure theory on sample-path spaces and the holonomy cocycles will also be given in Section 2, On the other hand, Section 3 is devoted to an analytic study on the holonomy cocycles. The proofs of Theorem 1.1 and Corollary 1.2 (modulo the integrability condition (1.1), i.e., Theorem 1.4) will be provided in Section 4 .

The remainder of the article is then devoted to the proof of inequality (1.1). This can be done in three reduction steps.

Section 5 collects several recent results about the mass-clustering of harmonic currents and a special parametrization of leaves near hyperbolic singularities.

The first reduction is carried out in Section 6. Namely, the proof of the integrability condition (1.1) is reduced to that of Theorem 6.2.

Section 7 lays the background for the geometric intersection of a harmonic current with an analytic curve defined on an open subset of $X$. We are inspired by Fornæss-Sibony's recent works in [14, 15, 16]. Special attention is focused on the case where the analytic curve is defined on a neighborhood of a singular 
point of the foliation. We also introduce the notion of interpretations: a way which permits us to estimate the mass of a geometric intersection efficiently.

In Section 8 we introduce test curves which consist of algebraic curves and analytic ones. The former curves are defined globally on $X$, whereas the latter ones are only defined on a neighborhood of a singular point of the foliation. Next, we state the first collection of basic estimates (see Propositions 8.3 , 8.4, 8.5) regarding the mass estimates of the geometric intersection of a harmonic current with test curves. This allows us to reduce the proof of Theorem 6.2 to those of Propositions 8.5 and 8.6 modulo Propositions 8.3 , 8.4. This is the second reduction.

Section 9 states the second collection of basic estimates (see Propositions 9.1 and 9.2). Next, using these estimate we establish a cohomological invariance result (see Proposition 9.3) which permits us to prove Proposition 8.5. So modulo Propositions $8.3,8.4,9.1,9.2$, the proof of Theorem 6.2 is finally reduced to that of Proposition 8.6 . This is the last reduction.

In Section 10 we study how the intersection points of test curves with a general leaf near singularities distribute. This analysis will be helpful when we want to estimate the mass of some geometric intersections in terms of interpretations. Based on this analysis, the remaining sections are then devoted to the proof of the above basic estimates (Propositions 8.3, 8.4, 9.1, 9.2 and 8.6).

Section 11 establishes Proposition 8.3 and the first half of Proposition 8.6.

Section 12 is devoted to the proof of Proposition 9.1.

Proposition 9.2 which consists of 3 basic estimates is proved in Section 13. The proof of each estimate occupies a whole subsection.

Finally, Section 14 completes the proof of the last half part of Proposition 8.6 as well as the proof of Proposition 8.4.

Acknowledgments. I would like to thank Nessim Sibony for suggesting me to work on the holonomy cocycle. Sincere thanks also go to Tien-Cuong Dinh for interesting discussions. I am also grateful to Mihai Paun and Jaigyoung Choe for very kind help, and to the referee for carefully reading the paper and for suggestions leading to the improvement of the exposition. The paper was partially prepared during my visit at Vietnam Institute for Advanced Study in Mathematics (VIASM) and at the Center for Mathematical Challenges (CMC) of the Korea Institute for Advanced Study (KIAS). I would like to express my gratitude to these organizations for hospitality and for financial support.

\section{BACKGROUND}

Although the main theorems only deal with complex surfaces as the ambient manifold $X$, we consider, in this section, the general case where $\operatorname{dim} X \geq 2$. Indeed, the section may serve as the background for the ongoing parts of the article. For a recent account on the theory of foliations, the reader is invited 
to consult the survey articles by Fornæss-Sibony [15], Ghys [18], Hurder [20] and textbooks by Candel-Conlon, Walczak [5, 6, 30].

Notation. Throughout the article, we denote by $\mathbb{D}$ the unit disc in $\mathbb{C}$. For $r>0$ we denote by $\mathbb{D}_{r}$ and $r \mathbb{D}$ interchangeably the disc in $\mathbb{C}$ with center 0 and with radius $r$. We use several notions of distances:

- dist denotes the distance on $X$ induced by the Hermitian metric $g_{X}$.

- $\operatorname{dist}_{P}$ denotes the Poincaré metric, it will be defined in Subsection 2.1, whereas a more elaborate variant of this distance will be considered in Section 3 .

- dist $_{C}$ denotes the compatible pseudo-distance, it will be defined in Section 10.

The current of integration on an analytic curve $\mathfrak{C}$ is denoted by $[\mathfrak{C}]$.

In this work the letters $c, c^{\prime}, c_{0}, c_{1}, c_{2}$ etc. denote positive constants, not necessarily the same at each occurrence. The notation $\gtrsim$ and $\lesssim$ means inequalities up to a multiplicative constant, whereas we write $\approx$ when both inequalities are satisfied. Let $O$ and $o$ denote the usual Landau asymptotic notations. Let $\log ^{\star}(\cdot):=1+|\log (\cdot)|$ be a log-type function.

2.1. Foliations, singularities, Poincaré metric and Brody hyperbolicity. Let $X$ be a complex manifold of dimension $k$. A holomorphic foliation by curves $\mathscr{F}=(X, \mathscr{L})$ on $X$ is the data of a foliation atlas $\mathscr{L}$ with charts

$$
\Phi_{p}: \mathbb{U}_{p} \rightarrow \mathbb{B}_{p} \times \mathbb{T}_{p} .
$$

Here, $\mathbb{T}_{p}$ is a domain in $\mathbb{C}^{k-1}, \mathbb{B}_{p}$ is a domain in $\mathbb{C}$, $\mathbb{U}_{p}$ is a domain in $X$, and $\Phi_{p}$ is biholomorphic, and all the changes of coordinates $\Phi_{p} \circ \Phi_{q}^{-1}$ are of the form

$$
x=(y, t) \mapsto x^{\prime}=\left(y^{\prime}, t^{\prime}\right), \quad y^{\prime}=\Psi(y, t), \quad t^{\prime}=\Lambda(t) .
$$

The open set $\mathbb{U}_{p}$ is called a flow box and the Riemann surface $\Phi_{p}^{-1}\{t=c\}$ in $\mathbb{U}_{p}$ with $c \in \mathbb{T}_{p}$ is a plaque. The property of the above coordinate changes insures that the plaques in different flow boxes are compatible in the intersection of the boxes. Two plaques are adjacent if they have non-empty intersection.

A leaf $L$ is a minimal connected subset of $X$ such that if $L$ intersects a plaque, it contains that plaque. So a leaf $L$ is a Riemann surface immersed in $X$ which is a union of plaques. A leaf through a point $x$ of this foliation is often denoted by $L_{x}$. A transversal is a complex submanifold of codimension 1 in $X$ which is transverse to the leaves of $\mathscr{F}$.

A holomorphic foliation by curves with singularities, or equivalently a singular holomorphic foliation by curves, is the data $(X, \mathscr{L}, E)$, where $X$ is a complex manifold, $E$ a closed subset of $X$ and $(X \backslash E, \mathscr{L})$ is a holomorphic foliation by curves. Each point in $E$ is said to be a singular point, and $E$ is said to be the set of singularities of the foliation. We always assume that $\overline{X \backslash E}=X$, see e.g. [10, 14] for more details. If $X$ is compact, then we say that the foliation $(X, \mathscr{L}, E)$ is compact. 
We say that a vector field $F$ on $\mathbb{C}^{k}$ is generic linear if it can be written as

$$
F(z)=\sum_{j=1}^{k} \lambda_{j} z_{j} \frac{\partial}{\partial z_{j}}
$$

where $\lambda_{j}$ are non-zero complex numbers. The integral curves of $F$ define a foliation on $\mathbb{C}^{k}$. The condition $\lambda_{j} \neq 0$ implies that the foliation has an isolated singularity at 0 . Consider a holomorphic foliation by curves $\mathscr{F}=(X, \mathscr{L}, E)$ with a discrete set of singularities $E$. We say that a singular point $x \in E$ is linearizable if there is a (local) holomorphic coordinate system of $M$ on an open neighborhood $\mathbb{U}_{x}$ of $x$ on which $x$ is identified with $0 \in \mathbb{C}^{k}$ and the leaves of $\mathscr{F}$ are integral curves of a generic linear vector field. Such neighborhood $\mathbb{U}_{x}$ is called a singular flow box of $x$. When $\operatorname{dim} X=k=2$, we say that a linearizable singular point $x \in E$ is hyperbolic if the associated generic linear vector field $F(z)=\lambda_{1} z_{1} \frac{\partial}{\partial z_{1}}+\lambda_{2} z_{2} \frac{\partial}{\partial z_{2}}$ satisfies $\lambda_{1} / \lambda_{2} \notin \mathbb{R}$. This property is independent of the choice of coordinates.

For the sake of simplicity, we adopt the following terminology throughout the article: Unless otherwise specified, a foliation means exactly a holomorphic foliation by curves $\mathscr{F}=(X, \mathscr{L}, E)$ in a Hermitian complex manifold $\left(X, g_{X}\right)$ with a (eventually empty) set $E$ of singularities.

Let $\mathscr{F}=(X, \mathscr{L}, E)$ be a foliation. We denote by $\mathscr{C}_{\mathscr{F}}$ the sheaf of functions $f$ defined and compactly supported on $X \backslash E$ which are leafwise smooth and transversally continuous, that is, for each foliation chart $\Phi_{p}: \mathbb{U}_{p} \rightarrow \mathbb{B}_{p} \times \mathbb{T}_{p}$ and all $m, n \in \mathbb{N}$, the derivatives $\frac{\partial^{m+n}\left(f \circ \Phi_{p}^{-1}\right)}{\partial y^{m} \partial \bar{y}^{n}}$ exist and are continuous in $(y, t)$.

Let $g_{P}$ be the Poincaré metric on the unit disc $\mathbb{D}$, defined by

$$
g_{P}(\zeta):=\frac{2}{\left(1-|\zeta|^{2}\right)^{2}} i d \zeta \wedge d \bar{\zeta}, \quad \zeta \in \mathbb{D}, \quad \text { where } i:=\sqrt{-1} .
$$

A leaf $L$ of the foliation is said to be hyperbolic if it is a hyperbolic Riemann surface, i.e., it is uniformized by $\mathbb{D}$. For a hyperbolic leaf $L_{x}$, let $\phi_{x}: \mathbb{D} \rightarrow L_{x}$ be a universal covering map with $\phi_{x}(0)=x$. Note that $\phi_{x}$ is unique up to a rotation around $0 \in \mathbb{D}$. Then, by pushing forward the Poincaré metric $g_{P}$ on $\mathbb{D}$ via $\phi_{x}$, we obtain the so-called Poincaré metric on $L_{x}$ which depends only on the leaf. The latter metric is given by a positive $(1,1)$-form on $L_{x}$ that we also denote by $g_{P}$ for the sake of simplicity. The foliation is said to be hyperbolic if its leaves are all hyperbolic.

For simplicity we still denote by $g_{X}$ the Hermitian metric on leaves of the foliation $(X \backslash E, \mathscr{L})$ induced by the ambient Hermitian metric $g_{X}$. Consider the function $\eta: X \backslash E \rightarrow[0, \infty]$ defined by

$$
\eta(x):=\sup \left\{\|D \phi(0)\|: \phi: \mathbb{D} \rightarrow L_{x} \text { holomorphic such that } \phi(0)=x\right\} .
$$

Here, for the norm of the differential $D \phi$ we use the Poincaré metric on $\mathbb{D}$ and the Hermitian metric $g_{X}$ on $L_{x}$. We record the following relation between $g_{X}$ 
and the Poincaré metric $g_{P}$ on leaves

$$
g_{X}=\eta^{2} g_{P}
$$

Recall from a recent joint-work with Dinh and Sibony [12] the following notion.

Definition 2.1. A foliation $\mathscr{F}=(X, \mathscr{L}, E)$ is said to be Brody hyperbolic if there is a constant $c>0$ such that $\eta(x) \leq c$ for all $x \in X \backslash E$.

Remark 2.2. It is clear that if the foliation is Brody hyperbolic then it is hyperbolic. Moreover, when $X$ is compact, the Brody hyperbolicity is equivalent to the non-existence of holomorphic non-constant maps $\mathbb{C} \rightarrow X$ such that out of $E$ the image of $\mathbb{C}$ is locally contained in a leaf, see [15, Theorem 15].

The following result is due to Lins Neto and Soares [24] (we only give the two-dimensional version although their result is also valid in $\mathbb{P}^{k}$ ):

Theorem 2.3. There exists a real Zariski dense open subset $\mathcal{S}(d)$ of the set of foliations with a given degree $d>1$ in $\mathbb{P}^{2}$ such that any $\mathscr{F} \in \mathcal{S}(d)$ satisfies

1) $\mathscr{F}$ has only hyperbolic singularities and no other singular points;

2) $\mathscr{F}$ has no invariant algebraic curve.

On the other hand, Brunella [2] has shown that each $\mathscr{F} \in \mathcal{S}(d)$ does not admit any holomorphic non-constant map $\mathbb{C} \rightarrow \mathbb{P}^{2}$ such that out of the singularities of $\mathscr{F}$ the image of $\mathbb{C}$ is locally contained in a leaf. Consequently, by Definition 2.1 and Remark 2.2, a generic holomorphic foliation in $\mathbb{P}^{2}$ with a given degree $d>1$ satisfies the hypotheses of Theorem 1.1, Corollaries 1.2 and 1.3 and Theorem 1.4 .

2.2. A local model. For $\mathbb{U}:=\mathbb{D}^{k}$ and $t>0$, let $t \mathbb{U}:=(t \mathbb{D})^{k}$, see Notation at the beginning of the section for the definition of $t \mathbb{D}$.

First we give a description of the local model for linearizable singularities. Consider the foliation $\left(\mathbb{D}^{k}, \mathscr{L},\{0\}\right)$ which is the restriction to $\mathbb{D}^{k}$ of the foliation associated to the vector field

$$
F(z)=\sum_{j=1}^{k} \lambda_{j} z_{j} \frac{\partial}{\partial z_{j}}
$$

with $\lambda_{j} \in \mathbb{C} \backslash\{0\}$. The foliation is singular at the origin. We use here the Euclidean metric on $\mathbb{D}^{k}$. Write $\lambda_{j}=s_{j}+i t_{j}$ with $s_{j}, t_{j} \in \mathbb{R}$. For $x=\left(x_{1}, \ldots, x_{k}\right) \in$ $\mathbb{D}^{k} \backslash\{0\}$, define the holomorphic map $\psi_{x}: \mathbb{C} \rightarrow \mathbb{C}^{k} \backslash\{0\}$ by

$$
\psi_{x}(\zeta):=\left(x_{1} e^{\lambda_{1} \zeta}, \ldots, x_{k} e^{\lambda_{k} \zeta}\right) \text { for } \zeta \in \mathbb{C}
$$

It is easy to see that $\psi_{x}(\mathbb{C})$ is the integral curve of $F$ which contains $\psi_{x}(0)=x$. Write $\zeta=u+i v$ with $u, v \in \mathbb{R}$. The domain $\Pi_{x}:=\psi_{x}^{-1}\left(\mathbb{D}^{k}\right)$ in $\mathbb{C}$ is defined by the inequalities

$$
s_{j} u-t_{j} v<-\log \left|x_{j}\right| \text { for } j=1, \ldots, k \text {. }
$$


So, $\Pi_{x}$ is a convex polygon which is not necessarily bounded. It contains 0 since $\psi_{x}(0)=x$. The leaf of $\mathscr{F}$ through $x$ contains the Riemann surface

$$
\widehat{L}_{x}:=\psi_{x}\left(\Pi_{x}\right) \subset L_{x} .
$$

In particular, the leaves in a singular flow box are parametrized using holomorphic maps $\psi_{x}: \Pi_{x} \rightarrow L_{x}$.

Now let $\mathscr{F}=(X, \mathscr{L}, E)$ be a Brody hyperbolic foliation on a Hermitian compact complex manifold $\left(X, g_{X}\right)$. Assume as usual that $E$ is finite and all points of $E$ are linearizable. Let dist be the distance on $X$ induced by the ambient metric $g_{X}$. We only consider flow boxes which are biholomorphic to $\mathbb{D}^{k}$. A regular flow box is a flow boxes outside the singularities. Singular flow boxes are identified to their models $\left(\mathbb{D}^{k}, \mathscr{L},\{0\}\right)$ as described above. For each singular point $x \in E$, we fix a singular flow box $\mathbb{U}_{x}$ such that $2 \mathbb{U}_{x} \cap 2 \mathbb{U}_{x^{\prime}}=\varnothing$ if $x, x^{\prime} \in E$ with $x \neq x^{\prime}$. We also cover $X \backslash \cup_{x \in E} \mathbb{U}_{x}$ by a finite number of regular flow boxes $\left(\mathbb{U}_{p}\right)_{p \in P}$ which are fine enough. In particular, each $\overline{\mathbb{U}}_{p}$ is contained in a larger regular flow box $2 \mathbb{U}_{p}$ with $2 \mathbb{U}_{p} \cap E=\varnothing$. Thus we obtain a finite cover $\mathscr{U}$ of $X$ consisting of regular flow boxes $\mathbb{U}_{p}$ and singular ones $\left(\mathbb{U}_{x}\right)_{x \in E}$. In this section we suppose that the ambient metric $g_{X}$ coincides with the standard Euclidean metric on each singular flow box $2 \mathbb{U}_{x} \simeq 2 \mathbb{D}^{k}, x \in E$. For $x=\left(x_{1}, \ldots, x_{k}\right) \in \mathbb{C}^{k}$, let $\|x\|$ be the standard Euclidean norm of $x$. Recall that $\log ^{\star}(\cdot):=1+|\log (\cdot)|$.

We record here the following crucial result which gives a precise estimate on the function $\eta$ introduced in (2.1).

Lemma 2.4. We keep the above hypotheses and notation. Then there exists a constant $c>1$ with the following properties.

1) $\eta \leq c$ on $X, \eta \geq c^{-1}$ outside the singular flow boxes $\cup_{x \in E} \frac{1}{4} \mathbb{U}_{x}$ and

$$
c^{-1} \cdot s \log ^{\star} s \leq \eta(x) \leq c \cdot s \log ^{\star} s
$$

for $x \in X \backslash E$ and $s:=\operatorname{dist}(x, E)$.

2) For every $x$ in a singular box which is identified with $\mathbb{D}^{k}$, for every $\zeta \in \Pi_{x}$,

$$
c^{-1} \cdot \frac{i d \zeta \wedge d \bar{\zeta}}{\left(\log ^{\star}\left(\psi_{x}(\zeta)\right)\right)^{2}} \leq\left(\psi_{x}^{*} g_{P}\right)(\zeta) \leq c \cdot \frac{i d \zeta \wedge d \bar{\zeta}}{\left(\log ^{\star}\left(\psi_{x}(\zeta)\right)\right)^{2}} .
$$

Proof. Part 1) has been proved in [12, Proposition 3.3].

To prove Part 2), write $y=\psi_{x}(\zeta)$ for $\zeta \in \Pi_{x}$, and observe that

$$
\min \left\{\left|\lambda_{1}\right|, \ldots,\left|\lambda_{k}\right|\right\} \cdot\|y\| \leq\left\|\psi_{x}^{\prime}(\zeta)\right\| \leq \max \left\{\left|\lambda_{1}\right|, \ldots,\left|\lambda_{k}\right|\right\} \cdot\|y\| .
$$

On the other hand, recall from (2.1) that

$$
i \partial \bar{\partial}\|y\|^{2}=\eta^{2}(y) g_{P}(y),
$$

Moreover, we know from Part 1 ) that $\eta(y) \approx\|y\| \log ^{\star}\|y\|$. Pulling back both members of the last equality by $\psi_{x}$ and using the previous estimates for $\left\|\psi_{x}^{\prime}(\zeta)\right\|$ and for $\eta(y)$, we obtain the desired estimate for $\left(\psi_{x}^{*} g_{P}\right)(\zeta)$. 
2.3. Heat diffusions and harmonic currents versus harmonic measures. Let $\mathscr{F}=(X, \mathscr{L}, E)$ be a hyperbolic foliation. The leafwise Poincaré metric $g_{P}$ induces the corresponding Laplacian $\Delta$ on leaves such that

$$
i \partial \bar{\partial} u=\Delta u \cdot g_{P}, \quad \text { on } X \backslash E \text { for all } u \in \mathscr{C}_{\mathscr{F}} .
$$

A positive finite Borel measure $\mu$ on $X$ is said to be harmonic if

$$
\int_{X} \Delta u d \mu=0
$$

for all functions $u \in \mathscr{C}_{\mathscr{F}}$.

For every point $x \in X \backslash E$, consider the heat equation on $L_{x}$

$$
\frac{\partial p(x, y, t)}{\partial t}=\Delta_{y} p(x, y, t), \quad \lim _{t \rightarrow 0} p(x, y, t)=\delta_{x}(y), \quad y \in L_{x}, t \in \mathbb{R}_{+} .
$$

Here $\delta_{x}$ denotes the Dirac mass at $x, \Delta_{y}$ denotes the Laplacian $\Delta$ with respect to the variable $y$, and the limit is taken in the sense of distribution, that is,

$$
\lim _{t \rightarrow 0+} \int_{L_{x}} p(x, y, t) f(y) g_{P}(y)=f(x)
$$

for every smooth function $f$ compactly supported in $L_{x}$.

The smallest positive solution of the above equation, denoted by $p(x, y, t)$, is called the heat kernel. Such a solution exists because $\left(L_{x}, g_{P}\right)$ is complete and of bounded geometry (see, for example, [6, 7]). The heat kernel gives rise to a one parameter family $\left\{D_{t}: t \geq 0\right\}$ of diffusion operators defined on bounded Borel measurable functions on $M \backslash E$ :

$$
D_{t} f(x):=\int_{L_{x}} p(x, y, t) f(y) g_{P}(y), \quad x \in X \backslash E .
$$

We record here the semi-group property of this family: $D_{0}=$ id and $D_{t+s}=$ $D_{t} \circ D_{s}$ for $t, s \geq 0$.

Let $\mathscr{C}_{\mathscr{F}}^{1}$ denote the space of forms $h$ of bidegree $(1,1)$ defined on leaves of the foliations such that $h$ is compactly supported on $X \backslash E$ and that $h$ is leafwise smooth and transversally continuous. A form $h \in \mathscr{C}_{\mathscr{F}}^{1}$ is said to be positive if its restriction to every plaque is a positive $(1,1)$-form in the usual sense of Lelong.

Definition 2.5. A harmonic current $T$ on the foliation $\mathscr{F}$ (or equivalently, directed by $\mathscr{F})$ is a linear continuous form on $\mathscr{C}_{\mathscr{F}}^{1}$ which verifies $\partial \bar{\partial} T=0$ in the weak sense (namely $T(\partial \bar{\partial} f)=0$ for all $f \in \mathscr{C}_{\mathscr{F}}$ ), and which is positive (namely, $T(h) \geq 0$ for all positive forms $h \in \mathscr{C}_{\mathscr{F}}^{1}$ ).

Suppose now that $E$ is a finite set. Then the existence of nonzero harmonic currents has been established by Berndtsson-Sibony in [1, Theorem 1.4], and Fornæss-Sibony in [15, Corollary 3]. The extension of $T$ by zero through $E$, still denoted by $T$, is a positive $\partial \bar{\partial}$-closed current on $X$. The total mass of the positive measure $T \wedge g_{X}$ is always finite.

We have the following decomposition (see [10, Proposition 2.3]). 
Proposition 2.6. Let $\mathscr{F}=(X, \mathscr{L}, E)$ be a hyperbolic foliation with linearizable singularities $E$. Let $T$ be a harmonic current on $X$. Let $\mathbb{U} \simeq \mathbb{B} \times \mathbb{T}$ be a flow box which is relatively compact in $X \backslash E$. Then, there is a positive Radon measure $\nu$ on $\mathbb{T}$ and for $\nu$-almost every $\alpha \in \mathbb{T}$ there is a positive harmonic function $h_{\alpha}$ on $\mathbb{B}$ such that if $K$ is compact in $\mathbb{B}$, the integral $\int_{\mathbb{T}}\left\|h_{\alpha}\right\|_{L^{1}(K)} d \nu(\alpha)$ is finite and

$$
\langle T, \chi\rangle=\int_{\mathbb{T}}\left(\int_{\mathbb{B}} h_{\alpha}(y) \chi(y, \alpha)\right) d \nu(t)
$$

for every form $\chi \in \mathscr{C}_{\mathscr{F}}^{1}$ compactly supported in $\mathbb{U}$.

A subset $M \subset X \backslash E$ is said to be leafwise saturated if $x \in M$ implies the whole leaf $L_{x}$ is contained in $M$. A positive finite measure $\mu$ on the $\sigma$-algebra of Borel sets in $X$ is said to be ergodic if for every leafwise saturated Borel measurable set $M \subset X, \mu(M)$ is equal to either $\mu(X)$ or 0 . A harmonic current $T$ is said to be extremal if it is an extremal point in the convex cone of all harmonic currents, i.e., if there are harmonic currents $T_{1}, T_{2}$ such that $T=\frac{T_{1}+T_{2}}{2}$, then $T_{1}$ and $T_{2}$ are colinear.

Theorem 2.7. Let $\mathscr{F}=(X, \mathscr{L}, E)$ be a hyperbolic foliation with linearizable singularities $E$.

1) The relation $\mu=T \wedge g_{P}$ is a one-to-one correspondence between the convex cone of harmonic currents $T$ and the convex cone of harmonic measures $\mu$.

2) If $T$ is extremal, then $\mu=T \wedge g_{P}$ is ergodic.

3) Each harmonic measure $\mu$ is $D_{t}$-invariant, i.e,

$$
\int_{X} D_{t} f d \mu=\int_{X} f d \mu, \quad f \in L^{1}(X, \mu) .
$$

Proof. We start with Part 1). First observe that, for each harmonic current $T$, the positive measure $\mu:=T \wedge g_{P}$ is finite by [10, Proposition 4.2]. Moreover, it is easy to see that $\mu$ is harmonic. Consequently, the map $T \mapsto T \wedge g_{P}$ is oneto-one. Therefore, to complete Part 1) it suffices to show that each harmonic measure $\mu$ may be written as $\mu=T \wedge g_{P}$ for some harmonic current $T$. To do this we proceed as in the proof of [10, Proposition 5.1].

To prove Part 2), suppose in order to get a contradiction that $\mu$ is not ergodic. So there is a leafwise saturated Borel set $A \subset X \backslash E$ such that $0<\mu(A)<1$. Let $\mu_{1}:=\left.2 \mu\right|_{A}$ and $\mu_{2}:=\left.2 \mu\right|_{X \backslash A}$. So $\mu=\frac{\mu_{1}+\mu_{2}}{2}$, and $\mu_{1}, \mu_{2}$ are not co-linear. Using the local description of $T$ on each flow box (see [10, Proposition 2.3]), we can show that both $\mu_{1}$ and $\mu_{2}$ are harmonic measures. By Part 1 ), let $T_{1}, T_{2}$ be harmonic currents such that $\mu_{1}:=T_{1} \wedge g_{P}$ and $\mu_{2}:=T_{2} \wedge g_{P}$. This, combined with $\mu=\frac{\mu_{1}+\mu_{2}}{2}$, implies that $T=\frac{T_{1}+T_{2}}{2}$ and $T_{1}, T_{2}$ are not co-linear. This contradicts the extremality of $T$.

Part 3) follows from [10, Theorem 6.4] applied to the positive (1,1)-form $\beta:=g_{P}$. 
2.4. Measure theory on sample-path spaces. In this subsection we follow the expositions given in Sections 2.2, 2.4 and 2.5 in [25] (see also [6]). The $\sigma$-algebra generated by a family $\mathscr{S}$ of subsets of $\Omega$ is, by definition, the smallest $\sigma$-algebra containing $\mathscr{S}$.

Let $\mathscr{F}=(X, \mathscr{L}, E)$ be a hyperbolic foliation endowed with the leafwise Poincaré metric $g_{P}$. Let $\Omega:=\Omega(\mathscr{F})$ be the space consisting of all continuous paths $\omega:[0, \infty) \rightarrow X$ with image fully contained in a single leaf. This space is called the sample-path space associated to $\mathscr{F}$. Observe that $\Omega$ can be thought of as the set of all possible paths that a Brownian particle, located at $\omega(0)$ at time $t=0$, might follow as time progresses. For each $x \in X \backslash E$, let $\Omega_{x}=\Omega_{x}(\mathscr{F})$ be the space of all continuous leafwise paths starting at $x$ in $X \backslash E$, that is,

$$
\Omega_{x}:=\{\omega \in \Omega: \omega(0)=x\} .
$$

Garnett developed in [17] a theory of leafwise Brownian motion in this context by constructing a $\sigma$-algebra $(\Omega, \widetilde{\mathscr{A}})$ together with a family of Wiener measures (see also [4, 6]). Now recall briefly her construction. A cylinder set (in $\Omega$ ) is a set of the form

$$
C=C\left(\left\{t_{i}, B_{i}\right\}: 1 \leq i \leq m\right):=\left\{\omega \in \Omega: \omega\left(t_{i}\right) \in B_{i}, \quad 1 \leq i \leq m\right\},
$$

where $m$ is a positive integer and the $B_{i}$ are Borel subsets of $X \backslash E$, and $0 \leq$ $t_{1}<t_{2}<\cdots<t_{m}$ is a set of increasing times. In other words, $C$ consists of all paths $\omega \in \Omega$ which can be found within $B_{i}$ at time $t_{i}$. For each point $x \in X \backslash E$, let

$$
W_{x}(C):=\left(D_{t_{1}}\left(\chi_{B_{1}} D_{t_{2}-t_{1}}\left(\chi_{B_{2}} \cdots \chi_{B_{m-1}} D_{t_{m}-t_{m-1}}\left(\chi_{B_{m}}\right) \cdots\right)\right)\right)(x),
$$

where $C:=C\left(\left\{t_{i}, B_{i}\right\}: 1 \leq i \leq m\right)$ as above, $\chi_{B_{i}}$ is the characteristic function of $B_{i}$ and $D_{t}$ is the diffusion operator given by $(2.5)$. Let $\widetilde{\mathscr{A}}=\widetilde{\mathscr{A}}(\mathscr{F})$ be the $\sigma$-algebra generated by all cylinder sets. It can be proved that $W_{x}$ extends to a probability measure on $(\Omega, \widetilde{\mathscr{A}})$.

In the recent work [25] we introduce another $\sigma$-algebra $\mathscr{A}$ on $\Omega$, which is bigger than $\widetilde{\mathscr{A}}$. In fact, $\mathscr{A}$ takes into account the holonomy phenomenon, whereas $\widetilde{\mathscr{A}}$ does not so. Here is our construction in the present context. The covering foliation $\widetilde{\mathscr{F}}=(\widetilde{X}, \widetilde{\mathscr{L}})$ of a singular foliation $\mathscr{F}$ is, in some sense, its universal cover. We give here its construction. For every leaf $L$ of $\mathscr{F}$ and every point $x \in L$, let $\pi_{1}(L, x)$ denotes the first fundamental group of all continuous closed paths $\gamma:[0,1] \rightarrow L$ based at $x$, i.e. $\gamma(0)=\gamma(1)=x$. Let $[\gamma] \in \pi_{1}(L, x)$ be the class of a closed path $\gamma$ based at $x$. Then the pair $(x,[\gamma])$ represents a point of $\widetilde{X}$. Thus the set of points $\widetilde{X}$ of $\widetilde{\mathscr{F}}$ is well-defined. The leaf $\widetilde{L}$ passing through a given point $(x,[\gamma]) \in \widetilde{X}$, is by definition, the set

$$
\widetilde{L}:=\left\{(y,[\delta]): y \in L_{x},[\delta] \in \pi_{1}(L, y)\right\},
$$


which is the universal cover of $L_{x}$. We put the following topological structure on $\widetilde{X}$ by describing a basis of open sets. Such a basis consists of all sets $\mathscr{N}(U, \alpha)$, $U$ being an open subset of $X \backslash E$ and $\alpha: U \times[0,1] \rightarrow X$ being a continuous function such that $\alpha_{x}:=\alpha(x, \cdot)$ is a closed path in $L_{x}$ based at $x$ for each $x \in U$, and

$$
\mathscr{N}(U, \alpha):=\left\{\left(x,\left[\alpha_{x}\right]\right): x \in U\right\} .
$$

The projection $\pi: \widetilde{X} \rightarrow X \backslash E$ is defined by $\pi(x,[\gamma]):=x$. It is clear that $\pi$ is locally homeomorphic and is a leafwise map. By pulling-back the foliation atlas $\mathscr{L}$ of $\mathscr{F}$ as well as the Poincaré metric $g_{P}$ via $\pi$, we obtain a natural foliation atlas $\widetilde{\mathscr{L}}$ for the hyperbolic foliation $\widetilde{\mathscr{F}}$ endowed with the leafwise metric $\pi^{*} g_{P}$. Denote by $\widetilde{\Omega}$ the sample-path space $\Omega(\widetilde{\mathscr{F}})$ associated with the foliation $\widetilde{\mathscr{F}}$.

Let $x \in X \backslash E$ and $\tilde{x}$ an arbitrary point in $\pi^{-1}(x) \subset \widetilde{X}$. Similarly as in (2.6), let $\widetilde{\Omega}_{\tilde{x}}=\Omega_{\tilde{x}}(\widetilde{\mathscr{F}})$ be the space of all paths in $\widetilde{\Omega}$ starting at $\tilde{x}$. Every path $\omega \in \Omega_{x}$ lifts uniquely to a path $\tilde{\omega} \in \widetilde{\Omega}_{\tilde{x}}$ in the sense that $\pi \circ \tilde{\omega}=\omega$. In what follows this bijective lifting is denoted by $\pi_{\tilde{x}}^{-1}: \Omega_{x} \rightarrow \widetilde{\Omega}_{\tilde{x}}$. So $\pi \circ\left(\pi_{\tilde{x}}^{-1}(\omega)\right)=\omega, \omega \in \Omega_{x}$.

Definition 2.8. Let $\mathscr{A}=\mathscr{A}(\mathscr{F})$ be the $\sigma$-algebra generated by all sets of following family

$$
\{\pi \circ \tilde{A}: \text { cylinder set } \tilde{A} \text { in } \widetilde{\Omega}\},
$$

where $\pi \circ \tilde{A}:=\{\pi \circ \tilde{\omega}: \tilde{\omega} \in \tilde{A}\}$.

Observe that $\widetilde{A} \subset \mathscr{A}$ and that the equality holds if every leaf of the foliation is homeomorphic to the disc $\mathbb{D}$. Now we construct a family $\left\{W_{x}\right\}_{x \in M \backslash E}$ of probability Wiener measures on $(\Omega, \mathscr{A})$. Let $x \in X \backslash E$ and $C$ an element of $\mathscr{A}$. Then we define the so-called Wiener measure $W_{x}$ by the following formula

$$
W_{x}(C):=W_{\tilde{x}}\left(\pi_{\tilde{x}}^{-1} C\right),
$$

where $\tilde{x}$ is an arbitrary point in $\pi^{-1}(x)$, and

$$
\pi_{\tilde{x}}^{-1} C:=\left\{\pi_{\tilde{x}}^{-1} \omega: \omega \in C \cap \Omega_{x}\right\},
$$

and $W_{\tilde{x}}$ is the probability measure on $(\widetilde{\Omega}, \widetilde{\mathscr{A}}(\widetilde{\mathscr{F}}))$ which was defined by (2.7). Given a positive finite Borel measure $\mu$ on $X \backslash E$, consider the measure $\bar{\mu}$ on $(\Omega, \mathscr{A})$ defined by

$$
\bar{\mu}(A):=\int_{X}\left(\int_{\omega \in A \cap \Omega_{x}} d W_{x}\right) d \mu(x), \quad A \in \mathscr{A} .
$$

The measure $\bar{\mu}$ is called the Wiener measure with initial distribution $\mu$. Here are its important properties.

Proposition 2.9. We keep the above hypotheses and notation.

(i) The value of $W_{x}(C)$ defined in (2.8) is independent of the choice of $\tilde{x}$. Moreover, $W_{x}$ is a probability measure on $(\Omega, \mathscr{A})$. 
(ii) $\bar{\mu}$ given in (2.9) is a positive finite measure on $(\Omega, \mathscr{A})$ and $\bar{\mu}(\Omega)=\mu(X \backslash E)$. (iii) If $\mu$ is harmonic, then $\bar{\mu}$ is time-invariant, that is,

$$
\int_{\Omega} F\left(\sigma_{t}(\omega)\right) d \bar{\mu}(\omega)=\int_{\Omega} F(\omega) d \bar{\mu}(\omega),
$$

for all $t \in \mathbb{R}^{+}$and $F \in L^{1}(\Omega, \bar{\mu})$, where the shift-transformation $\sigma_{t}: \Omega \rightarrow \Omega$ is defined by

$$
\sigma_{t}(\omega)(s):=\omega(s+t), \quad \omega \in \Omega, s \in \mathbb{R}^{+} .
$$

Proof. Assertion (i) has been proved in [25, Theorem 2.15]. Assertion (ii) has been established in [25, Theorem 2.16].

By Part 3) of Theorem [2.7, $\mu$ is $D_{t}$-invariant for all $t \in \mathbb{R}^{+}$. Consequently, applying [25, Theorem 2.20] to $\mu$ yields that $\bar{\mu}$ is time-invariant.

2.5. Holonomy cocycles. Now we define the holonomy cocycle of a hyperbolic foliation $\mathscr{F}=(X, \mathscr{L}, E)$ on a Hermitian complex surface $X$. For each point $x \in X \backslash E$, let $T_{x}(X)$ (resp. $T_{x}\left(L_{x}\right) \subset T_{x}(X)$ ) be the tangent space of $X$ (resp. $L_{x}$ ) at $x$. For every transversal $S$ at a point $x$ (that is, $x \in S$ ), let $T_{x}(S)$ denote the tangent space of $S$ at $x$.

Now fix a point $x \in X \backslash E$ and a path $\omega \in \Omega_{x}$ and a time $t \in \mathbb{R}^{+}$, and let $y:=\omega(t)$. Fix a transversal $S_{x}$ at $x$ (resp. $S_{y}$ at $y$ ) such that the complex line $T_{x}\left(S_{x}\right)$ is the orthogonal complement of the complex line $T_{x}\left(L_{x}\right)$ in the Hermitian space $\left(T_{x}(X), g(x)\right)$ (resp. $T_{y}\left(S_{y}\right)$ is the orthogonal complement of $T_{y}\left(L_{y}\right)$ in $\left.\left(T_{y}(X), g(y)\right)\right)$. Let $\operatorname{hol}_{\omega, t}$ be the holonomy map along the path $\left.\omega\right|_{[0, t]}$ from an open neighborhood of $x$ in $S_{x}$ onto an open neighborhood of $y$ in $S_{y}$. The derivative $D \operatorname{hol}_{\omega, t}: T_{x}\left(S_{x}\right) \rightarrow T_{y}\left(S_{y}\right)$ induces the so-called holonomy cocycle $\mathcal{H}: \Omega \times \mathbb{R}^{+} \rightarrow \mathbb{R}^{+}$given by

$$
\mathcal{H}(\omega, t):=\left\|D \operatorname{hol}_{\omega, t}(x)\right\| .
$$

The last map depends only on the path $\left.\omega\right|_{[0, t]}$, in fact, it depends only on the homotopy class of this path. In particular, it is independent of the choice of transversals $S_{x}$ and $S_{y}$. We see easily that

$$
\mathcal{H}(\omega, t)=\lim _{z \rightarrow x, z \in S_{x}} \operatorname{dist}\left(\operatorname{hol}_{\omega, t}(z), y\right) / \operatorname{dist}(z, x) .
$$

On the other hand, we note the following additive property which is an immediate consequence of the definition of $\mathcal{H}(\omega, t)$ (see also [25, Proposition 3.3]): (2.11)

$$
\log \|\mathcal{H}(\omega, t+s)\|=\log \|\mathcal{H}(\omega, t)\|+\log \left\|\mathcal{H}\left(\sigma_{t}(\omega), s\right)\right\|, \quad t, s \in \mathbb{R}^{+}, \omega \in \Omega,
$$

where $\sigma_{t}: \Omega \rightarrow \Omega$ is the shift-transformation given by (2.10). 


\section{HOLONOMY COCYCle VS POINCARÉ METRIC}

In this section let $\mathscr{F}=(X, \mathscr{L}, E)$ be a holomorphic Brody hyperbolic foliation with linearizable singularities $E$ in a Hermitian compact complex surface $X$. Let $\mathcal{H}$ be the holonomy cocycle of the foliation. In order to study the behavior of $\mathcal{H}$ near a (hyperbolic) singular point, we use the local model $\left(\mathbb{D}^{2}, \mathscr{L},\{0\}\right)$ introduced in Subsection 2.2. This is the restriction to $\mathbb{D}^{2}$ of the foliation associated with the vector field

$$
F(z, w)=z \frac{\partial}{\partial z}+\lambda w \frac{\partial}{\partial w} \quad \text { with some complex number } \quad \lambda \neq 0 .
$$

Since the main results of the article do not depend on the choice of a Hermitian metric on $X$, we can fix a metric which is equal to the Euclidean one in each singular flow box. This will simplify our presentation.

For $x=(z, w) \in \mathbb{D}^{2} \backslash\{0\}$, the holomorphic map $\psi_{x}: \Pi_{x} \rightarrow \mathbb{D}^{2} \backslash\{0\}$ given by (2.2) may be rewritten as

$$
\psi_{x}(\zeta):=\left(z e^{\zeta}, w e^{\lambda \zeta}\right) \quad \text { for } \quad \zeta \in \Pi_{x}
$$

Proposition 3.1. Let $\mathbb{D}^{2}$ be endowed with the Euclidean metric. For each $x=$ $(z, w) \in \mathbb{D}^{2}$ and $\zeta \in \Pi_{x}$, consider a path $\omega \in \Omega$ (if it exists) such that

$$
\omega(t)=\psi_{x}(t \zeta)=\left(z e^{\zeta t}, w e^{\lambda \zeta t}\right) \subset \mathbb{D}^{2}
$$

for all $t \in[0,1]$ (see (3.1) above). Then

$$
\mathcal{H}(\omega, 1)=\left|e^{\zeta}\right|\left|e^{\lambda \zeta}\right| \frac{\sqrt{|z|^{2}+|\lambda w|^{2}}}{\sqrt{\left|z e^{\zeta}\right|^{2}+\left|\lambda w e^{\lambda \zeta}\right|^{2}}} .
$$

Proof. Let $y:=\omega(1)=\left(z e^{\zeta}, w e^{\lambda \zeta}\right)$. Since the vector $(z, \lambda w)$ is tangent to the leaf $L_{x}$ at $x$, the vector $N_{x}:=(-\bar{\lambda} \bar{w}, \bar{z})$ is normal to $L_{x}$ at $x$, and hence, the complex normal line $S_{x}$ to $L_{x}$ at $x$ is the set

$$
\left\{x+s \cdot N_{x}: s \in \mathbb{C}\right\}=\{(z-\bar{\lambda} \bar{w} s, w+\bar{z} s): s \in \mathbb{C}\} .
$$

Similarly, let $N_{y}:=\left(-\bar{\lambda} \bar{w} e^{\bar{\lambda} \bar{\zeta}}, \bar{z} e^{\bar{\zeta}}\right)$ be the vector normal to $L_{y}$ at $y$, and let $S_{y}:=\left\{y+s \cdot N_{y}: s \in \mathbb{C}\right\}$ be the complex normal line to $L_{y}$ at $y$. Since $N_{x}$ (resp. $N_{y}$ ) may be regarded, in a sufficiently small open neighborhood of $x$ (resp. $y$ ), as a transversal, we can describe the holonomy map hol ${ }_{\omega, t}$ using them. Indeed, for each $s \in \mathbb{C}$ with $|s|$ small enough, we want to find $\xi \in \mathbb{C}$ close to $\zeta$ such that $\left((z-\bar{\lambda} \bar{w} s) e^{\xi},(w+\bar{z} s) e^{\lambda \xi}\right)$ belongs to $S_{y}$. This is equivalent to the fact that the following two vectors

$$
V_{s}:=\left((z-\bar{\lambda} \bar{w} s) e^{\xi}-z e^{\zeta},(w+\bar{z} s) e^{\lambda \xi}-w e^{\lambda \zeta}\right) \text { and } N_{y}
$$

are colinear. Write $\xi=\zeta+a s+O\left(s^{2}\right)$. So $e^{\xi}=e^{\zeta}\left(1+a s+O\left(s^{2}\right)\right)$ and $e^{\lambda \xi}=$ $e^{\lambda \zeta}\left(1+\lambda a s+O\left(s^{2}\right)\right)$. In order to determine $a$, we insert the last two identities 
into the expression of $V_{s}$ and get that

$$
V_{s}=s \cdot\left((z a-\bar{\lambda} \bar{w}) e^{\zeta},(\bar{z}+a \lambda w) e^{\lambda \zeta}\right)+O\left(s^{2}\right) .
$$

So the above colinearity condition reduces to the colinearity of the following two vectors

$$
\left((z a-\bar{\lambda} \bar{w}) e^{\zeta},(\bar{z}+a \lambda w) e^{\lambda \zeta}\right) \text { and }\left(-\bar{\lambda} \bar{w} e^{\bar{\lambda} \bar{\zeta}}, \bar{z} e^{\bar{\zeta}}\right) .
$$

Solving this equation yields that

$$
a=\frac{\bar{\lambda} \bar{z} \bar{w}\left(\left|e^{\zeta}\right|^{2}-\left|e^{\lambda \zeta}\right|^{2}\right)}{|z|^{2}\left|e^{\zeta}\right|^{2}+|\lambda|^{2}|w|^{2}\left|e^{\lambda \zeta}\right|^{2}} .
$$

Recall that $T_{x}\left(S_{x}\right)=S_{x}$ is orthogonal to $T_{x}\left(L_{x}\right)$ at $x=\omega(0)$ and $T_{y}\left(S_{y}\right)=S_{y}$ is orthogonal to $T_{y}\left(L_{y}\right)$ at $y=\omega(1)$. Moreover, $x+s \cdot N_{x}$ and $y+V_{s}$ are on the same leaf for all $s \in \mathbb{C}$ with $|s|$ small enough. Consequently, a geometric argument shows that

$$
\mathcal{H}(\omega, 1)=\lim _{s \rightarrow 0}\left\|V_{s}\right\| /\left\|s \cdot N_{s}\right\|=\frac{\left\|\left((z a-\bar{\lambda} \bar{w}) e^{\zeta},(\bar{z}+a \lambda w) e^{\lambda \zeta}\right)\right\|}{\|(-\bar{\lambda} \bar{w}, \bar{z})\|},
$$

where the last equality holds by (3.2). Inserting the above value of $a$ into the last expression, a straightforward calculation gives the desired result.

Now we define a new variant of Poincaré "distance" dist $_{P}$ which takes into account the holonomy phenomenon. Let $\omega \in \Omega$ and $0 \leq t \leq s$. Put $x:=\omega(t)$ and $y:=\omega(s)$. Let $\phi_{x}: \mathbb{D} \rightarrow L_{x}$ be a universal covering map with $\phi_{x}(0)=x$. The path $[0, s-t] \ni r \mapsto \omega(t+r)$ is lifted by $\phi_{x}$ to a continuous path $\beta:[0, s-t] \rightarrow \mathbb{D}$ such that $\beta(0)=0$. Let $\tau:=\beta(s-t) \in \mathbb{D}$. So $\phi_{x}(\tau)=\omega(s)=y$. Now we are in the position to define the new Poincaré function

$$
\operatorname{dist}_{P}(\omega: t, s):=\operatorname{dist}_{P}(0, \tau)=\log \left(\frac{1+|\tau|}{1-|\tau|}\right),
$$

where on the right hand side $\operatorname{dist}_{P}$ is the usual Poincaré distance on $\mathbb{D}$. Note that $\operatorname{dist}_{P}(\omega: t, s)$ is independent of the choice of $\phi_{x}$. Moreover, it is uniquely determined by $x=\omega(t), y=\omega(s)$ and the homotopy class (two end-points being fixed) of the path $[0, s-t] \ni r \mapsto \omega(t+r)$. There is exactly one homotopy class for which $\operatorname{dist}_{P}(\cdot: t, s)$ coincides with $\operatorname{dist}_{P}(x, y)$.

The following lemma shows us how deep a leaf can go into a singular flow box before the hyperbolic time $R$.

Lemma 3.2. There is a constant $c>0$ with the following property. Let $\omega \in \Omega$ be such that $\omega[0,1] \subset(1 / 2 \mathbb{D})^{2}$ and that $\omega[0,1]$ is (locally) geodesic with respect to the leafwise Poincaré metric $g_{P}$. Write $(z, w):=x=\omega(0)$ and $R:=\operatorname{dist}_{P}(\omega: 0,1)$. Then there exists $\zeta \in \Pi_{x}$ (see (3.1) above) such that $\omega(1)=\left(z e^{\zeta}, w e^{\lambda \zeta}\right)$ and that

$$
|\zeta| \leq e^{c R}|\log \|x\|| \text {. }
$$


Proof. First we show that there is $r>0$ such that if $R=\operatorname{dist}_{P}(\omega: 0,1) \leq r$ then there exists $\zeta \in \Pi_{x}$ such that $\omega(1)=\left(z e^{\zeta}, w e^{\lambda \zeta}\right)$ and that

$$
|\zeta| \leq \frac{|\log \|x\||}{2|\lambda|}
$$

Indeed, let $\omega \in \Omega$ be a path such that

- $\omega[0,1]$ is locally geodesic;

- for all $t \in[0,1], \omega(t):=\left(z e^{\zeta(t)}, w e^{\lambda \zeta(t)}\right) \in(1 / 2 \mathbb{D})^{2}$;

- $\zeta(0)=0$ and $|\zeta(t)| \leq \frac{|\log \|x\||}{2|\lambda|}$ for all $t \in[0,1]$ and $|\zeta(1)|=\frac{|\log \|x\||}{2|\lambda|}$.

We only need to show that $R=\operatorname{dist}_{P}(\omega: 0,1) \leq r$ for some $r>0$ independent of $\omega$. Indeed, it follows from the second and third $\bullet$ above that $|\log \|\omega(t)\|| \approx$ $|\log \|x\||$ for $t \in[0,1]$. Therefore, by integrating along the path $[0,1] \ni t \mapsto \zeta(t)$ and using the first $\bullet$ above, and applying Part 2) of Lemma 2.4, we get that

$$
\begin{aligned}
\operatorname{dist}_{P}(\omega: 0,1)=\int_{\omega[0,1]} \sqrt{g_{P}(z)}=\int_{0}^{1} \zeta_{t}^{*}\left(\psi_{x}^{*}\left(\sqrt{g_{P}}\right)\right) & \\
& \geq c_{1} \int_{0}^{\frac{|\log \|x\||}{2|\lambda|}}|\log \|x\||^{-1} d s=\frac{c_{1}}{2 \lambda}=: r,
\end{aligned}
$$

where $c_{1}>0$ is a constant. This proves (3.4).

Next, we prove the lemma for a general $R>0$. Suppose without loss of generality that $r=1$. Let $0=t_{0}<\cdots<t_{n}=1$ be a subdivision of $[0,1]$ such that $\operatorname{dist}_{P}\left(\omega: t_{j}, t_{j+1}\right) \leq 1$ for each $0 \leq j \leq n-1$ and that $n$ is as smallest as possible. So $n$ is the smallest integer $\geq R$. Let $x_{j}:=\omega\left(t_{j}\right)$. So $x_{0}=\omega(0)=x=$ $(z, w)$. Applying (3.4) repeatedly, we obtain, for each $0 \leq j \leq n, \zeta_{j} \in \mathbb{C}$ and $x_{j}=\left(z_{j}, w_{j}\right) \in(1 / 2 \mathbb{D})^{2}$ such that $x_{j+1}=\left(z_{j} e^{\zeta_{j}}, w_{j} e^{\lambda \zeta_{j}}\right)$ for $0 \leq j<n$ and that $\left|\zeta_{j}\right| \leq c_{2}\left|\log \left\|x_{j}\right\|\right|$. So $\left|\log \left\|x_{j+1}\right\|\right| \leq c_{3}\left|\log \left\|x_{j}\right\|\right|$ for some constant $c_{3}>1$ which depends only on $c_{2}$ and $\lambda$. Thus,

$$
\left|\log \left\|x_{j}\right\|\right| \leq c_{3}^{j}|\log \|x\|| \quad \text { and } \quad\left|\zeta_{j}\right| \leq c_{2} c_{3}^{j}|\log \|x\|| .
$$

Writing $\omega(1)=x_{n}=\left(z_{0} e^{\zeta}, w_{0} e^{\lambda \zeta}\right)=\left(z e^{\zeta}, w e^{\lambda \zeta}\right)$ with $\zeta:=\zeta_{1}+\cdots+\zeta_{n-1}$ and using the last estimate, the desired conclusion of the lemma follows.

The following result gives an estimate on the expansion rate of $\mathcal{H}(\omega, \cdot)$ in terms of the Poincaré function $\operatorname{dist}_{P}(\omega: \cdot, \cdot)$ and the distance dist $(\omega(0), E)$.

Proposition 3.3. There is a constant $c>0$ such that

$$
|\log \|\mathcal{H}(\omega, t)\|| \leq c \log ^{\star} \operatorname{dist}(\omega(0), E) \cdot \exp \left(c \operatorname{dist}_{P}(\omega: 0, t)\right), \quad \omega \in \Omega, t \in \mathbb{R}^{+} .
$$

Proof. We may suppose without loss of generality that $t=1$. Let $\omega \in \Omega$, and put $x:=\omega(0)$ and $y:=\omega(1)$. Since $\mathcal{H}(\omega, 1)$ depends only on the homotopy class of the path $\left.\omega\right|_{[0,1]}$, we may assume without loss of generality that the segment $\omega[0,1]$ is (locally) geodesic with respect to the Poincaré metric on $L_{x}$. Let $\mathscr{U}$ be the finite cover of $M$ by regular and singular flow boxes given in Subsection 
2.2. We consider three steps.

Step 1: If there is a singular flow box $\mathbb{U}$ which contains the whole segment $\omega([0,1])$, then the proposition is true for $c=c_{1}$, where $c_{1}>0$ is a constant large enough.

Write $x=(z, w)$ and $y:=\omega(1)$. Let $R:=\operatorname{dist}_{P}(\omega: 0,1)$. By Lemma 3.2, we may write $y=\left(z e^{\zeta}, w e^{\lambda \zeta}\right)$ for some $\zeta \in \mathbb{C}$ such that

$$
|\zeta| \leq e^{c_{2} R}
$$

Inserting this into the expression for the holonomy map given in Proposition 3.1, a straightforward computation shows that

$$
|\log \|\mathcal{H}(\omega, 1)\|| \leq c_{3}|\log \|x\|| e^{c_{3} R}
$$

for a constant $c_{3}>0$ independent of $\omega$. Choosing $c_{1}>c_{3}$ large enough, Step 1 follows from the last estimate.

Step 2: If the whole segment $\omega([0,1])$ is contained in a single regular flow box $\mathbb{U} \in \mathscr{U}$, then $|\log \|\mathcal{H}(\omega, 1)\|| \leq c_{4}$, where $c_{4}>0$ is a constant independent of $\omega$. In particular, the proposition is true in this case for $c=c_{1}$, where $c_{1}>0$ is a constant large enough.

Observe that the geodesic segment $\omega[0,1]$ is contained in the unique plaque of $\mathbb{U}$ which passes through $x$. This, combined with the description of the holonomy map on $\mathbb{U}$, implies that $\|\mathcal{H}(\omega, 1)\| \leq e^{c_{4}}$ for a constant $c_{4}>0$ independent of $\omega$. Hence, $|\log \|\mathcal{H}(\omega, 1)\|| \leq c_{4}$. Therefore, choosing $c_{1}>c_{4}$ large enough, we have that

$$
c_{1} \log ^{\star} \operatorname{dist}(\omega(0), E) \geq c_{4} \geq|\log \|\mathcal{H}(\omega, 1)\|| .
$$

This proves the proposition in Step 2.

Step 3: Proof of the proposition in the general case.

Consider the family of all finite subdivisions of $[0,1]$ into intervals $\left[t_{j-1}, t_{j}\right]$ with $1 \leq j \leq n$ such that $t_{0}=0, t_{n}=1$ and that each segment $\omega\left(\left[t_{j-1}, t_{j}\right]\right)$ is contained in a single (regular or singular) flow box $\mathbb{U}_{j}$ for each $j$. Fix a member of this family such that the number $n$ is smallest possible. We may assume without loss of generality that $n>1$ since the case $n=1$ follows either from Step 1 (if $\mathbb{U}_{1}$ is singular) or from Step 2 (if $\mathbb{U}_{1}$ is regular). The minimality of $n$ implies that all $\omega\left(t_{1}\right), \ldots \omega\left(t_{n-1}\right)$ belong to the union of all regular flow boxes of $\mathscr{U}$. Therefore, there is a constant $r_{0}>0$ independent of $\omega$ such that

$$
\operatorname{dist}_{P}\left(\omega: t_{j}, t_{j+1}\right) \geq r_{0}, \quad 1 \leq j \leq n-1 .
$$

Thus

$$
n \leq 1+r_{0}^{-1} \operatorname{dist}_{P}(\omega: 0,1) .
$$

Moreover, there is a constant $c_{5}>1$ independent of $\omega$ such that

$$
1 \leq \log ^{\star} \operatorname{dist}\left(\omega\left(t_{j}\right), E\right) \leq c_{5}, \quad 1 \leq j \leq n-1 .
$$


Using this and applying Step 1 to each singular box in the family $\left(\mathbb{U}_{j}\right)_{j=1}^{n}$ and applying Step 2 to each regular flow box in the above family, we obtain that

$$
\begin{aligned}
\left|\log \left\|\mathcal{H}\left(\omega, t_{1}\right)\right\|\right| & \leq c_{1} \log ^{\star} \operatorname{dist}\left(\omega\left(t_{0}\right), E\right) \cdot \exp \left(c_{1} \operatorname{dist}_{P}\left(\omega: t_{0}, t_{1}\right)\right), \\
\left|\log \| \mathcal{H}\left(\sigma_{t_{j-1}}(\omega), t_{j}-t_{j-1}\right)\right| & \leq c_{1} c_{5} \exp \left(c_{1} \operatorname{dist}_{P}\left(\omega: t_{j-1}, t_{j}\right)\right), \quad 2 \leq j \leq n .
\end{aligned}
$$

Summing up the above estimates, we get that

$$
\begin{array}{r}
\sum_{j=1}^{n}\left|\log \left\|\mathcal{H}\left(\sigma_{t_{j-1}}(\omega), t_{j}-t_{j-1}\right)\right\|\right| \leq c_{1} \log ^{\star} \operatorname{dist}\left(\omega\left(t_{0}\right), E\right) \cdot \exp \left(c_{1} \operatorname{dist}_{P}\left(\omega: t_{0}, t_{1}\right)\right) \\
+\sum_{j=2}^{n} c_{1} c_{5} \exp \left(c_{1} \operatorname{dist}_{P}\left(\omega: t_{j-1}, t_{j}\right)\right) .
\end{array}
$$

On the other hand, we infer from (2.11) that

$$
|\log \|\mathcal{H}(\omega, 1)\||=\sum_{j=1}^{n}\left|\log \left\|\mathcal{H}\left(\sigma_{t_{j-1}}(\omega), t_{j}-t_{j-1}\right)\right\|\right| .
$$

This, coupled with the previous estimate, gives that

$$
\begin{aligned}
|\log \|\mathcal{H}(\omega, 1)\|| \leq c_{1} \log ^{\star} \operatorname{dist}\left(\omega\left(t_{0}\right), E\right) \cdot \exp \left(c_{0} \operatorname{dist}_{P}\left(\omega: t_{0}, t_{1}\right)\right) \\
+\sum_{j=2}^{n} c_{1} c_{5} \exp \left(c_{1} \operatorname{dist}_{P}\left(\omega: t_{j-1}, t_{j}\right)\right) .
\end{aligned}
$$

Since $\log ^{\star} \operatorname{dist}(x, E) \geq 1$ for all $x \in M \backslash E$, the right hand side of the last line is dominated by a constant times $\log ^{\star} \operatorname{dist}\left(\omega\left(t_{0}\right), E\right)$ times

$$
\sum_{j=1}^{n} \exp \left(c_{1} \operatorname{dist}_{P}\left(\omega: t_{j-1}, t_{j}\right)\right) \leq n \cdot \exp \left(c_{1} \operatorname{dist}_{P}(\omega: 0,1)\right),
$$

where the last inequality holds because of the identity

$$
\operatorname{dist}_{P}(\omega: 0,1)=\sum_{j=1}^{n} \operatorname{dist}_{P}\left(\omega: t_{j-1}, t_{j}\right) .
$$

Inserting (3.5) into the right hand side of the last inequality and choosing $c>c_{1}$ large enough, we find that its left hand side is bounded by $c \exp \left(c \operatorname{dist}_{P}(\omega: 0,1)\right)$. So the right hand side of (3.6) is also bounded by a constant times $\log ^{\star} \operatorname{dist}\left(\omega\left(t_{0}\right), E\right)$. $\exp \left(c \operatorname{dist}_{P}(\omega: 0,1)\right)$, and the proof is thereby completed. 


\section{PROOF OF THE MAIN RESULTS MODULO THE INTEGRABILITY CONDITION}

This section is devoted to the proofs of Theorem 1.1 and Corollary 1.2 modulo the integrability condition (1.1), i.e., modulo Theorem 1.4. We need the following result.

Lemma 4.1. There is a constant $c>1$ such that for all $x \in M \backslash E$ and all $s \geq 1$,

$$
W_{x}\left\{\omega \in \Omega: \sup _{t \in[0,1]} \operatorname{dist}_{P}(\omega: 0, t)>s\right\}<c e^{-c^{-1} s^{2}} .
$$

Proof. Let $\phi_{x}: \mathbb{D} \rightarrow L_{x}$ be a universal covering map with $\phi_{x}(0)=x$. We have to show that

$$
W_{0}\left\{\omega \in \Omega(\mathbb{D}): \sup _{t \in[0,1]} \operatorname{dist}_{P}(\omega(0), \omega(t))>s\right\}<c e^{-c^{-1} s^{2}}
$$

where $W_{0}$ is the Wiener measure at 0 of the unit disc $\mathbb{D}$ endowed with the Poincaré metric $g_{P}$, and $\operatorname{dist}_{P}(\cdot, \cdot)$ is the Poincaré distance. Since the Poincaré metric is complete and of bounded geometry, the last estimate holds by combining [4, Lemma 8.16 and Corollary 8.8].

Now we arrive at the End of the proof of Theorem 1.1 modulo the integrability condition (1.1). By Proposition 3.3, we get a constant $c_{1}>0$ such that

$$
\mathscr{I}(\omega) \leq c_{1} \mathcal{G}(\omega)
$$

where the function $\mathcal{G}: \Omega \rightarrow \mathbb{R}^{+}$is given by

$$
\mathcal{G}(\omega):=\log ^{\star} \operatorname{dist}(\omega(0), E) \cdot \exp \left(c_{1} \cdot \sup _{t \in[0,1]} \operatorname{dist}_{P}(\omega: 0, t)\right), \quad \omega \in \Omega
$$

Consequently, we only need to show that $\mathcal{G}$ is $\bar{\mu}$-integrable.

To do this we write using formula (2.9)

$$
\int_{\Omega} \mathcal{G}(\omega) d \bar{\mu}(\omega)=\int_{X} \log ^{\star} \operatorname{dist}(x, E) \cdot\left(\int_{\Omega_{x}} \exp \left(c_{1} \cdot \sup _{t \in[0,1]} \operatorname{dist}_{P}(\omega: 0,1)\right) d W_{x}(\omega)\right) d \mu(x) .
$$

Next, we will show that the inner integral is uniformly bounded by a constant $c_{2}>0$ independent of $x$, that is,

$$
\int_{\Omega_{x}} \exp \left(c_{1} \cdot \sup _{t \in[0,1]} \operatorname{dist}_{P}(\omega: 0,1)\right) d W_{x}(\omega)<c_{2} .
$$


To this end we focus on a single leaf $L$ of $\mathscr{F}$ passing through a given point $x \in X \backslash E$. Observe that

$$
\begin{aligned}
\int_{\Omega_{x}} \exp \left(c_{1} \cdot \sup _{t \in[0,1]} \operatorname{dist}_{P}(\omega: 0,1)\right) d W_{x}(\omega) \\
\quad=\int_{0}^{\infty} W_{x}\left\{\omega \in \Omega_{x}: \exp \left(c_{1} \cdot \sup _{t \in[0,1]} \operatorname{dist}_{P}(\omega: 0,1)\right)>s\right\} d s .
\end{aligned}
$$

The integrand on the right-hand side is equal to

$$
W_{x}\left\{\omega \in \Omega_{x}: \sup _{t \in[0,1]} \operatorname{dist}_{P}(\omega: 0,1)>\log s / c_{1}\right\} .
$$

For $0 \leq s \leq e^{c_{1}}$, this quantity is clearly $\leq 1$ since $W_{x}$ is a probability measure by Proposition 2.9 (i). For $s \geq e^{c_{1}}$, this quantity is dominated, thanks to Lemma 4.1, by $c_{3} \exp \left(-c_{3}^{-1}\left(\frac{\log s}{c_{1}}\right)^{2}\right)$ for some constant $c_{3}>0$. Since $\int_{e^{c_{1}}}^{\infty} \exp \left(-c_{3}^{-1}\left(\frac{\log s}{c_{1}}\right)^{2}\right) d s<$ $\infty$, we have established (4.2).

We infer from (4.1) and (4.2) that

$$
\int_{\Omega} \mathcal{G}(\omega) d \bar{\mu}(\omega) \leq c_{2} \int_{X} \log ^{\star} \operatorname{dist}(x, E) d \mu(x) .
$$

By assumption (1.1), the integral on the right hand is is finite. Hence, the proof of the theorem is complete.

End of the proof of Corollary 1.2 modulo the integrability condition (1.1). Using Theorem 1.1, we may apply [25, Theorem 3.7] to the holonomy cocycle $\mathcal{H}$ of rank 1. Consequently, we obtain a unique Lyapunov exponent function $\lambda(T): \quad X \rightarrow \mathbb{R}$ which is measurable and leafwise constant and which, for $\mu$-almost every $x \in X$, satisfies

$$
\lim _{t \rightarrow \infty} \frac{1}{t} \log \|\mathcal{H}(\omega, t)\|=\lambda(T)(x)
$$

for $W_{x}$-almost every path $\omega \in \Omega_{x}$. Since $\mu$ is ergodic and the function $\lambda(T)$ is leafwise constant and measurable, it follows that for all $a, b \in \mathbb{R}$ with $a \leq b$, the $\mu$-measure of the leafwise saturated set $\{x \in X: a \leq \lambda(T)(x) \leq b\}$ is either 0 or $\mu(X)$. Consequently, $\lambda(T)$ is constant $\mu$-almost everywhere. The proof is thereby completed.

\section{HARMONIC CURRENTS ON THE LOCAL MODEL}

We collect in this section several known results about the mass-clustering of harmonic measures near hyperbolic singularities. More concretely, we first recall a special parametrization of leaves which is due to Fornæss-Sibony [16]. Next, using this parametrization, we state a mass-clustering result of harmonic measures near hyperbolic singularities which is also due to Fornæss-Sibony 
[16]. Finally, we recall our recent estimate about the behaviour of some integral operators of "Poisson kernel" type near hyperbolic singularities. These results will thoroughly be used in the subsequent sections when we prove the basic estimates stated in Section 6 .

Following [16, Section 2], consider the foliation associated to the vector field $F(z, w)=z \frac{\partial}{\partial z}+\lambda w \frac{\partial}{\partial w}$ with some complex number $\lambda=a+i b, b \neq 0$. Note that if we flip $z$ and $w$, we replace $\lambda$ by $1 / \lambda=\bar{\lambda} /|\lambda|^{2}=a /\left(a^{2}+b^{2}\right)-i b /\left(a^{2}+\right.$ $b^{2}$ ). Therefore, we may assume without loss of generality that $b>0$. We now describe the portion of a general leaf inside $\mathbb{D}^{2}$. There are two separatrices, $(w=0),(z=0)$. Other than that the Riemann surface $\widehat{L}_{\alpha}$ defined in (2.3) can be reparametrized by

$$
(z, w)=\psi_{\alpha}(\zeta), z=e^{i(\zeta+(\log |\alpha|) / b)}, \zeta=u+i v, w=\alpha e^{i \lambda(\zeta+(\log |\alpha|) / b)} .
$$

The reader is invited to compare this special parametrization with the ones given in (2.3) and (3.1). Consider the new variable

$$
t:=b u+a v \text {. }
$$

So we have

$$
|z|=e^{-v},|w|=e^{-b u-a v}=e^{-t} .
$$

Observe that as we follow $z$ once counterclockwise around the origin, $u$ increases by $2 \pi$, so the absolute value of $|w|$ decreases by the multiplicative factor of $e^{-2 \pi b}$. Hence, we cover all leaves when $\alpha$ ranges over $\mathbb{T}$, where

$$
\mathbb{T}:=\left\{\alpha \in \mathbb{C}: e^{-2 \pi b} \leq|\alpha| \leq 1\right\} .
$$

We notice that with the above parametrization, the intersection with the unit bidisc $\mathbb{D}^{2}$ of the leaf is given by the domain $\left\{(u, v) \in \mathbb{R}^{2}: v>0, u>-a v / b\right\}$. The main point of this special parametrization is that the above domain is independent of $\alpha$. In the $(u, v)$-plane this domain corresponds to a sector $S_{\lambda}$ with corner at 0 and given by $0<\theta<\arctan (-b / a)$ where the $\arctan$ is chosen to have values in $(0, \pi)$, that is,

$$
S_{\lambda}:=\left\{\tau=r e^{i \theta} \in \mathbb{C}: r>0 \text { and } 0<\theta<\arctan (-b / a)\right\} .
$$

Let $\gamma:=\frac{\pi}{\arctan (-b / a)}$. Then the map

$$
\phi: \tau=u+i v \mapsto \tau^{\gamma}=(u+i v)^{\gamma}=: U+i V
$$

maps this sector to the upper half plane with coordinates $(U, V)$. The fact that $\gamma>1$ will be crucial, this is where the hyperbolicity of singularities is used.

The local leaf clusters on both separatrices. To investigate the clustering on the $z$-axis, we use a transversal $\mathbb{T}_{z_{0}}:=\left\{\left(z_{0}, w\right): e^{-2 \pi b} \leq|w| \leq 1\right\}$ for some $z_{0}$ with $\left|z_{0}\right|=1$. We can normalize so that $h_{\alpha}\left(z_{0}, w\right)=1$ for $\left(z_{0}, w\right) \in \mathbb{T}_{z_{0}}$. Solving the equation $\left(z_{0}, w\right)=\psi_{\alpha}\left(\zeta_{0}\right)=\psi_{\alpha}\left(u_{0}+i v_{0}\right)$ with unknown variables $(u, v, \alpha)$ yields the unique solution $u_{0}=-b^{-1} \ln |w|, v_{0}=0$ and $\alpha=w$. Consequently, by identifying $\alpha \in \mathbb{T}$ with $\left(z_{0}, \alpha\right) \in \mathbb{T}_{z_{0}}$, we may identify $\mathbb{T}$ with $\mathbb{T}_{z_{0}}$, and hence 
$\mathbb{T}$ can be regarded as a transversal. We call $\mathbb{T}$ the distinguished transversal. Let $T$ be a harmonic current of mass 1 directed by $\mathscr{F}$. Let $\mathbb{U}$ be a flow box which admits $\mathbb{T}_{z_{0}}$ as a transversal. Then by Proposition 2.6, we can write in $\mathbb{U}$

$$
T=\int h_{\alpha}\left[V_{\alpha}\right] d \nu(\alpha)
$$

where, for each $\alpha \in \mathbb{T}, h_{\alpha}$ denotes the harmonic function associated to the current $T$ on the plaque $V_{\alpha}$ which is contained in the leaf $L_{\alpha}$. We still denote by $h_{\alpha}$ its harmonic continuation along $L_{\alpha}$. Define

$$
\tilde{h}_{\alpha}(\zeta):=h_{\alpha}\left(e^{i(\zeta+(\log |\alpha|) / b)}, \alpha e^{i \lambda(\zeta+(\log |\alpha|) / b)}\right) \quad \text { on } \quad S_{\lambda} .
$$

Consider the harmonic function

$$
\tilde{H}_{\alpha}:=\tilde{h}_{\alpha} \circ \phi^{-1} \quad \text { defined on the upper half plane } \quad\{U+i V: V>0\} .
$$

The following mass-clustering estimate of Fornaess-Sibony [16] is needed.

Lemma 5.1. 1) The harmonic function $\tilde{H}_{\alpha}$ is the Poisson integral of its boundary values. So in the upper half plane $\{U+i V: V>0\}$,

$$
\tilde{H}_{\alpha}(U+i V)=\frac{1}{\pi} \int_{-\infty}^{\infty} \tilde{H}_{\alpha}(y) \frac{V}{V^{2}+(y-U)^{2}} d y
$$

for $\nu$-almost every $\alpha$. Moreover,

$$
\int_{\alpha \in \mathbb{T}} \int_{-\infty}^{\infty} \tilde{H}_{\alpha}(y)(1+|y|)^{1 / \gamma-1} d y d \nu(\alpha)<\infty .
$$

2) If, moreover, $T$ gives no mass to every invariant analytic curve, then $\nu$ is diffuse, that is, $\nu(\alpha)=0$ for every $\alpha$.

Proof. The first part is proved in [16, Proposition 1].

When $\mathscr{F}$ has no invariant analytic curve, the second part is proved in [16, Corollary 2]. But that proof still works in the more general context of Part 2) making the obviously necessary changes.

\section{Proof of THE INTEgRABILITY CONDITION: FIRST REDUCTION}

In this section we reduce Theorem 1.4 to Theorem 6.2, Let $\mathscr{F}=(X, \mathscr{L}, E)$ be a holomorphic hyperbolic foliation with hyperbolic singularities $E$ in a compact complex projective surface $X$ such that the foliation is Brody hyperbolic. Let $T$ be a harmonic current tangent to $\mathscr{F}$. Fix $x_{0} \in E$. Since $x_{0}$ is a hyperbolic singular point, there is a holomorphic coordinate system $(z, w)$ near $x_{0}$ in which $x_{0}$ is identified with 0 and the foliation $\mathscr{F}$ is associated with the vector field $F(z, w)=z \frac{\partial}{\partial z}+\lambda w \frac{\partial}{\partial w}$ on $\mathbb{D}^{2}$ with some complex number $\lambda=a+i b, b>0$. So two analytic curves $\{z=0\}$ and $\{w=0\}$ describe two separatrices of $\mathscr{F}$ at 
$x_{0}=0$. Let $\mathbb{T}$ be the distinguished transversal defined in (5.4). Consider the function $G: E \times(0,1) \rightarrow \mathbb{R}^{+}$given by

$$
G(x, r):=\frac{1}{2 \pi r^{2}} \int_{\mathbb{B}(x, r)} T \wedge i \partial \bar{\partial}\|y\|^{2},
$$

where $\mathbb{B}(x, r)$ is the ball of center $x$ and radius $r$ in $X$. By Skoda [28], $G(x, r)$ is increasing in $r$ and $\lim _{r \rightarrow 0} G(x, r)$ is equal to the Lelong number of $T$ at $x$. By our recent work [27], this number is 0 , that is,

$$
\lim _{r \rightarrow 0} G(x, r)=0 .
$$

When $x=x_{0}$, we write $G(r)$ instead of $G\left(x_{0}, r\right)$. Using the above map $\Psi$, we are reduced to the local model considered in the previous section. For every $s>0$, consider the function $K_{s}: \mathbb{R} \rightarrow \mathbb{R}^{+}$given by

$$
K_{s}(y):= \begin{cases}s^{1-\gamma}, & \text { if } s \geq(1+|y|)^{1 / \gamma} \\ (1+|y|)^{1 / \gamma-1}, & \text { if } s \leq(1+|y|)^{1 / \gamma} .\end{cases}
$$

The following result gives a precise estimate of $G(r)$ in terms of the function $K_{s}$.

Lemma 6.1. There is a constant $c>0$ such that for every $0<r<1$, we have

$$
c^{-1} G(r) \leq \int_{\alpha \in \mathbb{T}}\left(\int_{-\infty}^{\infty} K_{-\log r}(y) \tilde{H}_{\alpha}(y) d y\right) d \nu(\alpha) \leq c G(r) .
$$

Proof. It follows from combining [27, Proposition 3.5] and [27, Lemma 3.2].

We are in the position to state the main estimate of this article.

Theorem 6.2. There are constants $c_{0}, \kappa>1$ such that for every $x \in E$ and $0<r<1 / 2$,

$G(x, r) \leq c_{0}|\log (-\log r)||\log r|^{-1}+c_{0} \int_{\alpha \in \mathbb{T}}\left(\int_{(1+|y|)^{1 / \gamma} \leq-\kappa \log r} K_{-\log r}(y) \tilde{H}_{\alpha}(y) d y\right) d \nu(\alpha)$.

The proof of Theorem 6.2 will be given at the end of Section 8 .

Remark 6.3. Using Proposition 8.3 below (for $\delta=1$ ), Theorem 6.2 is equivalent to the assertion that

$$
\begin{aligned}
\int_{\mathbb{B}(0, r)} T \wedge[z=r] \leq c_{0} \mid & \left.\log (-\log r)|| \log r\right|^{-1} \\
& +c_{0} \int_{\alpha \in \mathbb{T}}\left(\int_{(1+|y|)^{1 / \gamma \leq-\kappa \log r}} K_{-\log r}(y) \tilde{H}_{\alpha}(y) d y\right) d \nu(\alpha) .
\end{aligned}
$$

This is the precise meaning of the speed that we mention in Subsection 1.2 (see the discussion following (1.4)). The integral on the right hand side of the 
last line decays, in some sense, very quickly as $r \rightarrow 0$. Indeed, it is, up to a multiplicative constant, equal to

$$
\int_{\alpha \in \mathbb{T}}\left(\int_{(1+|y|)^{1 / \gamma} \leq-\kappa \log r}(-\log r)^{1-\gamma} \tilde{H}_{\alpha}(y) d y\right) d \nu(\alpha) .
$$

Rewrite the last line as follows:

$$
\int_{\alpha \in \mathbb{T}}\left(\int_{(1+|y|)^{1 / \gamma} \leq-\kappa \log r} \frac{(1+|y|)^{1-1 / \gamma}}{(-\log r)^{\gamma-1}}(1+|y|)^{1 / \gamma-1} \tilde{H}_{\alpha}(y) d y\right) d \nu(\alpha) .
$$

Since for every $y \in \mathbb{R}, \frac{(1+|y|)^{1-1 / \gamma}}{(-\log r)^{\gamma-1}} \rightarrow 0$ as $r \rightarrow 0$, it follows from Lemma 5.1 and the dominated convergence that the last integral tends to 0 as $r \rightarrow 0$ (see [27] for details).

Taking for granted this result, we arrive at the End of the proof of Theorem 1.4. Fix a point $x_{0} \in E$ and a holomorphic coordinate system $x=(z, w)$ as at the beginning of this section. So $x_{0}$ is identified with $0 \in \mathbb{D}^{2}$. Since the two Hermitian metrics $g_{X}$ and $i \partial \bar{\partial}\|x\|^{2}$ are equivalent on $\mathbb{D}^{2}$, that is, $g_{X} \approx i \partial \bar{\partial}\|x\|^{2}$, we may regard $i \partial \bar{\partial}\|x\|^{2}$ as $g_{X}$. Moreover, in the remainder of the proof, we will write $\mathbb{B}_{r}$ (resp. $G(r)$ ) instead of $\mathbb{B}\left(x_{0}, r\right)$ (resp. $\left.G\left(x_{0}, r\right)\right)$ for $0<r<1$. Next, recall from (2.1) that

$$
i \partial \bar{\partial}\|x\|^{2}=\eta^{2}(x) g_{P}(x),
$$

where we know from Part 1) of Lemma 2.4 that $\eta(x) \approx\|x\| \log \|x\|$ for $0<$ $\|x\|<1 / 2$. Therefore, we infer that

$$
\mu:=T \wedge g_{P} \approx \frac{T \wedge i \partial \bar{\partial}\|x\|^{2}}{\|x\|^{2}(\log \|x\|)^{2}} \quad \text { on } \mathbb{B}_{1 / 2} .
$$

Moreover, we infer from (6.1) that for every smooth function $h:[0,1] \rightarrow \mathbb{R}^{+}$,

$$
\int_{\mathbb{B}_{1 / 2}} \frac{T \wedge i \partial \bar{\partial}\|x\|^{2}}{h(\|x\|)}=\int_{0}^{1 / 2} \frac{d\left(r^{2} G(r)\right)}{h(r)} .
$$

Consequently,

$$
\int_{\mathbb{B}_{1 / 2}}\left|\log ^{\star} \operatorname{dist}(x, E)\right| \cdot d \mu(x) \approx \int_{\mathbb{B}_{1 / 2}} \frac{T \wedge i \partial \bar{\partial}\|x\|^{2}}{\|x\|^{2}(\log \|x\|)}=\int_{0}^{1 / 2} \frac{d\left(r^{2} G(r)\right)}{-r^{2} \log r} .
$$

Performing an integration by part to the last expression yields that

$$
\begin{aligned}
\int_{0}^{1 / 2} \frac{d\left(r^{2} G(r)\right)}{-r^{2} \log r} & =\left[\frac{G(r)}{-\log r}\right]_{0}^{1 / 2} \\
& -2 \int_{0}^{1 / 2} \frac{G(r) d r}{r \log r}-\int_{0}^{1 / 2} \frac{G(r) d r}{r(\log r)^{2}} .
\end{aligned}
$$


Since $G(r)$ tends to the Lelong number of $T$ at 0 as $t \rightarrow 0$, the expression in brackets is finite. Therefore, in order to show that $\int_{\mathbb{B}_{1 / 2}}\left|\log ^{\star} \operatorname{dist}(x, E)\right| \cdot d \mu(x)<$ $\infty$, it suffices to prove that

$$
\int_{0}^{1 / 2} \frac{G(r) d r}{-r \log r}<\infty
$$

The remaining part is devoted to the proof of (6.4). By Theorem 6.2, the integral in (6.4) is bounded by a constant times $(I)+(I I)$, where

$$
I:=\int_{0}^{1 / 2} \frac{|\log (-\log r)| d r}{r|\log r|^{2}}<\infty,
$$

and by Fubini's theorem,

$$
I I:=\int_{\alpha \in \mathbb{T}}\left(\int_{y=-\infty}^{\infty}\left(\int_{(1+|y|)^{1 / \gamma} \leq-\kappa \log r} \frac{K_{-\log r}(y) d r}{-r \log r}\right) \tilde{H}_{\alpha}(y) d y\right) d \nu(\alpha) .
$$

On the other hand, we infer from (6.3) the existence of a constant $c>0$ such that for all $y \in \mathbb{R}$,

$$
\int_{s \geq \kappa^{-1}(1+|y|)^{1 / \gamma}} s^{-1} K_{s}(y) d s \leq c(1+|y|)^{1 / \gamma-1} .
$$

Performing the change of variable $s:=-\log r$ in the last line, the most inner integral of $(I I)$ is dominated by a constant times $(1+|y|)^{1 / \gamma-1}$. Consequently, $(I I)$ is bounded by

$$
\int_{\alpha \in \mathbb{T}} \int_{-\infty}^{\infty} \tilde{H}_{\alpha}(y)(1+|y|)^{1 / \gamma-1} d y d \nu(\alpha),
$$

which is finite by Part 1) of Lemma 5.1. This completes the proof of (6.4), and hence the proof of the theorem.

Remark 6.4. As remarked in the Introduction, the method employed in DinhNguyen-Sibony [10] seems to only give a weaker inequality

$$
\int\left|\log ^{\star} \operatorname{dist}(x, E)\right|^{1-\delta} \cdot\left(T \wedge g_{P}\right)(x)<\infty, \quad \delta>0 .
$$

Indeed, arguing as in the proof of Theorem 1.4 and using the weight $\left|\log ^{\star} \operatorname{dist}(x, E)\right|^{1-\delta}$ instead of $\left|\log ^{\star} \operatorname{dist}(x, E)\right|$, the above inequality is reduced to the following one

$$
\int_{0}^{1 / 2} \frac{G(r) d r}{-r(\log r)^{1+\delta}}<\infty .
$$

In [10] $G(r)$ is replaced by a positive constant, and hence the above integral is finite if and only if $\delta>0$. 


\section{GEOMETRIC INTERSECTION AND INTERPRETATIONS}

Let $\mathscr{F}=(X, \mathscr{L}, E)$ be a holomorphic hyperbolic foliation with hyperbolic singularities $E$ in a compact complex surface $X$. Let $T$ be a harmonic current tangent to $\mathscr{F}$, and let $\mathfrak{C}$ be an analytic curve on an open subset $\mathbb{U} \subset X$. The main purpose of the section is to give a reasonable meaning to the intersection measure $T \wedge[\mathfrak{C}]$, and to obtain a procedure in order to estimate the mass of the last measure. We are inspired by the recent works in [14, 15, 16].

Let $\mathbb{U} \simeq \mathbb{B} \times \mathbb{T}$ be a flow box which is relatively compact in $X \backslash E$. Let $\mathfrak{C}$ be an analytic curve on $\mathbb{U}$ such that for every $\alpha \in \mathbb{T}, \mathfrak{C}$ intersects the plaque $V_{\alpha}$ at at most one point (which is possibly a multiple point). We say that $\mathfrak{C}$ is transversal in $\mathbb{U}$. We define the geometric intersection of $T$ and $[\mathfrak{C}]$ as the positive Radon measure on $\mathbb{U}$ given by:

$$
\langle T \wedge[\mathfrak{C}], \phi\rangle=\left.\langle T \wedge[\mathfrak{C}], \phi\rangle\right|_{\mathbb{U}}:=\int_{\alpha \in \mathbb{T}: \xi_{\alpha} \neq \varnothing} h\left(\xi_{\alpha}\right) \phi\left(\xi_{\alpha}\right) d \nu(\alpha),
$$

where $\phi$ is a continuous test function compactly supported in $\mathbb{U}$, and

- $\xi_{\alpha}:=V_{\alpha} \cap \mathfrak{C}$ if this intersection is non empty and $\xi_{\alpha}=\varnothing$ otherwise;

- the decomposition consisting of the positive Radon measure $\nu$ on $\mathbb{T}$, and the positive harmonic function $h_{\alpha}$ on $\mathbb{B}$ for $\nu$-almost every $\alpha \in \mathbb{T}$ is given by Proposition 2.6.

The reader can easily check the following result.

Proposition 7.1. $T \wedge[\mathfrak{C}]$ is a well-defined positive Radon measure on $\mathbb{U}$. It is independent of the choice of a decomposition given by Proposition 2.6 Its mass is

$$
\|T \wedge[\mathfrak{C}]\|=\|T \wedge[\mathfrak{C}]\|_{\mathbb{U}}=\int_{\alpha \in \mathbb{T}: \xi_{\alpha} \neq \varnothing} h\left(\xi_{\alpha}\right) d \nu(\alpha)<\infty .
$$

Now let $\mathbb{U}$ be an an arbitrarily open subset of $X$ and $\mathfrak{C}$ an analytic curve on $\mathbb{U}$. We say that $\mathfrak{C}$ is almost transversal in $\mathbb{U}$ if $\mathfrak{C}$ intersects with each plaque in every regular flow box in $\mathbb{U}$ transversally at at most finite points. We leave the reader to verify the following result.

Lemma 7.2. $\mathfrak{C}$ is almost transversal if and only if $\mathfrak{C}$ is locally transversal in $\mathbb{U}$, that is, for every $x \in \mathfrak{C} \cap \mathbb{U}$, there is a flow box $\mathbb{U}_{x} \subset \mathbb{U}$ containing $x$ such that $\mathfrak{C}$ is transversal in $\mathbb{U}_{x}$.

Assume that $\mathfrak{C}$ is almost transversal. By Lemma 7.2, there is an at most countable cover $\mathscr{U}:=\left(\mathbb{U}_{j}\right)_{j \in J}$ of $\mathbb{U} \backslash E$ by its open subsets such that $\mathscr{U}$ is locally finite and that each $\mathbb{U}_{j}(j \in J)$ is a flow box which is relatively compact in $\mathbb{U} \backslash E$ and that $\mathfrak{C}$ is transversal in $\mathbb{U}_{j}$. Let $\Theta:=\left(\theta_{j}\right)_{j \in J}$ be a partition of unity subordinate to $\mathscr{U}$.

The mass of the intersection $T \wedge[\mathfrak{C}]$ is

$$
\|T \wedge[\mathfrak{C}]\|=\left.\sum_{j \in J}\left\langle T \wedge[\mathfrak{C}], \theta_{j}\right\rangle\right|_{\mathbb{U}_{j}} \in[0, \infty] .
$$


Apparently, the mass $\|T \wedge[\mathfrak{C}]\|$ depends on the choice of a cover $\mathscr{U}$ and a partition of unity $\Theta$. However, it turns out that this mass is independent of such a choice. More precisely, we can show the following properties.

Proposition 7.3. $\quad$ (i) The mass $\|T \wedge[\mathfrak{C}]\|$ does not depend on any choice we made.

(ii) If $\mathbb{U} \cap E=\varnothing$, then $\|T \wedge[\mathfrak{C}]\|<\infty$.

(iii) When $\|T \wedge[\mathfrak{C}]\|<\infty$, we define the geometric intersection of $T$ and $[\mathfrak{C}]$ as the positive Radon measure on $\mathbb{U}$ given by:

$$
\langle T \wedge[\mathfrak{C}], \phi\rangle:=\sum_{j \in J}\left\langle T \wedge[\mathfrak{C}], \theta_{j} \phi\right\rangle_{\mathbb{U}_{j}},
$$

where $\phi$ is a continuous test function compactly supported in $\mathbb{U}$,

(iv) When $\|T \wedge[\mathfrak{C}]\|<\infty$, the measure $T \wedge[\mathfrak{C}]$ defined by (7.2) does not depend on any choice of $\mathscr{U}$ and $\Theta$ we made.

Next, we prove a cohomological invariant property.

Proposition 7.4. Let $\mathfrak{C}$ and $\mathfrak{D}$ be two algebraic curves on $X$ which are cohomologous (in the cohomology group $H^{1,1}(X, \mathbb{R})$ ). Suppose that $\mathfrak{C} \cap E=\mathfrak{D} \cap E=\varnothing$ and that both $\mathfrak{C}$ and $\mathfrak{D}$ are almost transversal. Then $\|T \wedge[\mathfrak{C}]\|_{X}=\|T \wedge[\mathfrak{D}]\|_{X}$.

Proof. Since $\mathfrak{C} \cap E=\mathfrak{D} \cap E=\varnothing$ and both $\mathfrak{C}$ and $\mathfrak{D}$ are almost transversal, we may find a finite cover $\mathscr{U}:=\left(\mathbb{U}_{j}\right)_{j \in J}$ of $X$ by its open subsets such that

- if $\mathbb{U}_{j} \cap E \neq \varnothing$, then this intersection is a single point and $\mathfrak{C} \cap \mathbb{U}_{j}=\mathfrak{D} \cap \mathbb{U}_{j}=\varnothing$;

- each $\mathbb{U}_{j}$ with $U_{j} \cap E=\varnothing$ is a regular flow box such that both $\mathfrak{C}$ and $\mathfrak{D}$ are transversal in $\mathbb{U}_{j}$. Let $\left(\theta_{j}\right)_{j \in J}$ be a partition of unity subordinate to $\mathscr{U}$.

Consider a smooth Hermitian metric $\|\cdot\|$ on the line bundle generated by the divisor $[\mathfrak{C}]$ (resp. $[\mathfrak{D}]$ ) on $X$. Let $\sigma$ (resp. $\sigma^{\prime}$ ) be a holomorphic section having $[\mathfrak{C}]$ (resp. $[\mathfrak{D}]$ ) as its divisor. Then

$$
\phi:=\log \|\sigma\| \quad \text { and } \quad \psi:=\log \left\|\sigma^{\prime}\right\|
$$

are quasi-plurisubharmonic functions on $X$. Recall here that a quasi-plurisubharmonic function is locally the sum of a plurisubharmonic function and a smooth one. Lelong-Poincaré formula says that

$$
[\mathfrak{C}]=i \partial \bar{\partial} \phi+\Theta \quad \text { and } \quad[\mathfrak{D}]=i \partial \bar{\partial} \psi+\Theta^{\prime},
$$

where $\Theta$ and $\Theta^{\prime}$ are some closed smooth real $(1,1)$-forms on $X$. Since $[\mathfrak{C}]$ and $[\mathfrak{D}]$ are cohomologous, it follows that so are $\Theta$ and $\Theta^{\prime}$. So by the $\partial \bar{\partial}$-lemma for compact Kähler manifolds, there is a smooth real function $u$ on $X$ such that $\Theta^{\prime}-\Theta=i \partial \bar{\partial} u$. Therefore, replacing the metric $\|\cdot\|$ on the line bundle associated with $[\mathfrak{C}]$ by $\|\cdot\| e^{-2 u}$, we may assume without loss of generality that $\Theta^{\prime}=\Theta$.

Observe that $\phi$ (resp. $\psi$ ) is smooth outside the curve $\mathfrak{C}$ (resp. $\mathfrak{D}$ ). Since $\mathfrak{C} \cap E=\mathfrak{D} \cap E=\varnothing$, we infer that both $\phi$ and $\psi$ are smooth in a neighborhood of $E$. 
Fix the following decreasing sequence (as $\epsilon \searrow 0$ ) of quasi-plurisubharmonic smooth functions $\left(\phi_{\epsilon}\right)_{0<\epsilon<1}$ (resp. $\left.\left(\psi_{\epsilon}\right)_{0<\epsilon<1}\right)$ on $X$ :

$$
\phi_{\epsilon}:=\frac{1}{2} \log \left(\|\sigma\|^{2}+\epsilon\right) \quad \text { and } \quad \psi:=\frac{1}{2} \log \left(\left\|\sigma^{\prime}\right\|^{2}+\epsilon\right) .
$$

Observe that

$$
\lim _{\epsilon \rightarrow 0+} \phi_{\epsilon}=\phi \quad \text { and } \quad \lim _{\epsilon \rightarrow 0+} \psi_{\epsilon}=\psi
$$

and that there is a closed smooth real $(1,1)$-form $\Xi$ on $X$ such that

$$
i \partial \bar{\partial} \phi_{\epsilon} \geq \Xi \quad \text { and } \quad i \partial \bar{\partial} \psi_{\epsilon} \geq \Xi
$$

in the sense of currents and independent of $\epsilon$.

If $\mathbb{U}_{j} \cap E \neq \varnothing$, we deduce from the first $\bullet$ above as well as the properties of $\phi$ and $\psi$ discussed above that $\phi_{\epsilon}$ (resp. $\psi_{\epsilon}$ ) converges uniformly to $\phi$ (resp. $\psi$ ) as $\epsilon \searrow 0$ on $\mathbb{U}_{j}$.

If $\mathbb{U}_{j} \cap E \neq \varnothing$, we need the following result whose proof will be given later on.

Lemma 7.5. For every $\mathbb{U}_{j} \simeq \mathbb{B}_{j} \times \mathbb{T}_{j} \in \mathscr{U}$ with $\mathbb{U}_{j} \cap E=\varnothing$, we have that

$$
\lim _{\epsilon \rightarrow 0} \sup _{\alpha \in \mathbb{T}}\left\|\phi_{\epsilon}-\phi\right\|_{L^{1}\left(V_{\alpha}\right)}=0 \quad \text { and } \quad \lim _{\epsilon \rightarrow 0} \sup _{\alpha \in \mathbb{T}}\left\|\psi_{\epsilon}-\psi\right\|_{L^{1}\left(V_{\alpha}\right)}=0 .
$$

Resuming the proof of Proposition 7.4, let $\chi$ be a continuous test function on $X$. In what follows we drop the index $j$ for simplicity, e.g. we will write $\mathbb{U} \simeq \mathbb{B} \times \mathbb{T}, \theta$ instead of $\mathbb{U}_{j} \simeq \mathbb{B}_{j} \times \mathbb{T}_{j}, \theta_{j}$ respectively. Let $\mathbb{U} \in \mathscr{U}$ be such that $\mathbb{U} \cap E=\varnothing$. Write $\mathbb{U} \simeq \mathbb{B} \times \mathbb{T}$. Using (7.3) and noting that $\Theta^{\prime}=\Theta$, and applying Lelong-Poincaré formula on each plaque $V_{\alpha}, \alpha \in \mathbb{T}$ of $\mathbb{U}$, we get that

$$
\begin{aligned}
h\left(\xi_{\alpha}\right)(\theta \chi)\left(\xi_{\alpha}\right) & =\left.\left\langle\left.\Theta\right|_{V_{\alpha}}+\left.i \partial \bar{\partial} \phi\right|_{V_{\alpha}}, h \theta \chi\right\rangle\right|_{V_{\alpha}} \\
& =\left.\left\langle\left.\Theta\right|_{V_{\alpha}} h \theta \chi\right\rangle\right|_{V_{\alpha}}+\left.\left\langle\left. i \partial \bar{\partial} \phi\right|_{V_{\alpha}}, h \theta \chi\right\rangle\right|_{V_{\alpha}} \\
& =\left.\left\langle\left.\Theta\right|_{V_{\alpha}} h \theta \chi\right\rangle\right|_{V_{\alpha}}+\left.\lim _{\epsilon \rightarrow 0}\left\langle\left. i \partial \bar{\partial} \phi_{\epsilon}\right|_{V_{\alpha}}, h \theta \chi\right\rangle\right|_{V_{\alpha}} \\
& =\left.\left\langle\left.\Theta\right|_{V_{\alpha}} h \theta \chi\right\rangle\right|_{V_{\alpha}}+\left.\lim _{\epsilon \rightarrow 0}\left\langle\left.\phi_{\epsilon}\right|_{V_{\alpha}}, i \partial \bar{\partial}(h \theta \chi)\right\rangle\right|_{V_{\alpha}},
\end{aligned}
$$

where the third equality holds since $\phi_{\epsilon} \rightarrow \phi$ weakly on $V_{\alpha}$, and the last equality is obtained by Stokes' theorem. Since (7.4) says that the last limit is uniform in $\alpha$, we can integrate both extreme sides of the last chain of equalities with 
respect to the measure $d \nu$ and obtain that

$$
\begin{aligned}
\langle T \wedge[\mathfrak{C}], \theta \chi\rangle_{\mathbb{U}} & =\int_{\alpha \in \mathbb{T}} h\left(\xi_{\alpha}\right)(\theta \chi)\left(\xi_{\alpha}\right) d \nu(\alpha) \\
& =\left.\int_{\alpha \in \mathbb{T}}\left\langle\left.\Theta\right|_{V_{\alpha}}, h \theta \chi\right\rangle\right|_{V_{\alpha}} d \nu(\alpha)+\left.\int_{\alpha \in \mathbb{T}} \lim _{\epsilon \rightarrow 0}\left\langle\phi_{\epsilon}, i \partial \bar{\partial}(h \theta \chi)\right\rangle\right|_{V_{\alpha}} d \nu(\alpha) \\
& =\langle T \wedge \Theta, \theta \chi\rangle+\left.\lim _{\epsilon \rightarrow 0} \int_{\alpha \in \mathbb{T}}\left\langle i \partial \bar{\partial} \phi_{\epsilon}, h \theta \chi\right\rangle\right|_{V_{\alpha}} d \nu(\alpha) \\
& =\langle T \wedge \Theta, \theta \chi\rangle+\lim _{\epsilon \rightarrow 0}\left\langle T \wedge i \partial \bar{\partial} \phi_{\epsilon}, \theta \chi\right\rangle .
\end{aligned}
$$

On the other hand, for $\mathbb{U} \in \mathscr{U}$ with $\mathbb{U} \cap E \neq \varnothing$, we have that

$$
\langle T \wedge[\mathfrak{C}], \theta \chi\rangle_{\mathbb{U}}=0=\langle T \wedge \Theta, \theta \chi\rangle+\lim _{\epsilon \rightarrow 0}\left\langle T \wedge i \partial \bar{\partial} \phi_{\epsilon}, \theta \chi\right\rangle,
$$

where we use the first $\bullet$ above and the fact that $\phi$ is smooth on a neighborhood of the support of $\theta \chi$.

Summing up the above equalities over all $\mathbb{U} \in \mathscr{U}$ and using Definition (7.2), we infer that

$$
\langle T \wedge[\mathfrak{C}], \chi\rangle=\langle T \wedge \Theta, \chi\rangle+\lim _{\epsilon \rightarrow 0}\left\langle T \wedge i \partial \bar{\partial} \phi_{\epsilon}, \chi\right\rangle .
$$

When $\chi \equiv 1$, the last equality becomes

$$
\|T \wedge[\mathfrak{C}]\|_{X}=\langle T, \Theta\rangle+\lim _{\epsilon \rightarrow 0}\left\langle T, i \partial \bar{\partial} \phi_{\epsilon}\right\rangle=\langle T, \Theta\rangle,
$$

where the last equality is obtained since $\left\langle T, i \partial \bar{\partial} \phi_{\epsilon}\right\rangle=0$ as $T$ is harmonic and $\phi_{\epsilon}$ is smooth on $X$. Hence, $\|T \wedge[\mathfrak{C}]\|_{X}=\langle T, \Theta\rangle$. Similarly, we also get that $\|T \wedge[\mathfrak{D}]\|_{X}=\langle T, \Theta\rangle$. The proof is thereby completed.

End of the proof of Lemma 7.5. We only need to show that

$$
\lim _{\epsilon \rightarrow 0} \sup _{\alpha \in \mathbb{T}}\left\|\phi_{\epsilon}-\phi\right\|_{L^{1}\left(V_{\alpha}\right)}=0
$$

since the other assertion can be proved similarly. Since $\mathbb{U}_{j} \cap E=\varnothing$, the second $\bullet$ above says that $\mathfrak{C}$ is transversal in $\mathbb{U}_{j}$. Therefore, we are reduced to the following model where $\mathbb{U}_{j} \simeq \mathbb{B}_{j} \times \mathbb{T}_{j} \simeq(1 / 2 \mathbb{D})^{2}$ and

$$
\mathfrak{C} \cap \mathbb{U}_{j}=\{(w, f(w)): \quad w \in 1 / 2 \mathbb{D}\},
$$

where $f: 1 / 2 \mathbb{D} \rightarrow 1 / 2 \mathbb{D}$ is a holomorphic function.

In this model, we see easily that modulo a smooth function $\phi_{\epsilon}(z, w)=\frac{1}{2} \log (\mid z-$ $\left.\left.f(w)\right|^{2}+\epsilon\right)$ for $(z, w) \in(1 / 2 \mathbb{D})^{2}$. So (7.5) becomes

$$
\sup _{w \in 1 / 2 \mathbb{D}} \int_{z \in 1 / 2 \mathbb{D}}\left(\frac{1}{2} \log \left(|z-f(w)|^{2}+\epsilon\right)-\log |z-f(w)|\right) i d z \wedge d \bar{z} \rightarrow 0 \quad \text { as } \quad \epsilon \searrow 0 .
$$

Since the right hand side is bounded from above by

$$
\int_{z \in \mathbb{D}} \frac{1}{2}\left(\log \left(|z|^{2}+\epsilon\right)-\log |z|\right) i d z \wedge d \bar{z}
$$


and that this integral converges to 0 as $\epsilon \searrow 0$, the desired estimate follows.

The rest of the section is devoted to the case when the foliation $\mathscr{F}$ on the open set $\mathbb{U}$ is holomorphically equivalent to the foliation associated with the vector field $F$ in $\mathbb{D}^{2}$ introduced in Section 5, So we are in the local model considered in Section 5 and $0 \in \mathbb{U}=\mathbb{D}^{2}$. We keep the notation introduced in Section 5 . Recall that $\mathbb{T} \simeq\left\{\alpha \in \mathbb{C}: e^{-2 \pi b} \leq|\alpha| \leq 1\right\}$. Let $\mathfrak{C}$ be an analytic curve on $\mathbb{D}^{2}$ which is locally transversal in $\mathbb{D}^{2}$. For every $\alpha \in \mathbb{T}$, let $\left\{\xi_{\alpha_{j}}: j \in J_{\alpha}\right\}$ be the set of all intersections of $\mathfrak{C}$ with the Riemann surface $\widetilde{L}_{\alpha}$. We make the following convention $J_{\alpha}:=\left\{0,1, \ldots, n_{\alpha}\right\}$ with $n_{\alpha} \in \mathbb{N} \cup\{\infty\}$. Continuing Proposition 7.3 we can prove the following result.

Proposition 7.6. $\quad$ (i) The following equality holds

$$
\|T \wedge[\mathfrak{C}]\|=\int_{\alpha \in \mathbb{T}} \sum_{j \in J_{\alpha}} h_{\alpha}\left(\xi_{\alpha, j}\right) d \nu(\alpha) .
$$

(ii) If $\|T \wedge[\mathfrak{C}]\|<\infty$, then the measure $T \wedge[\mathfrak{C}]$ can be extended to a continuous linear form on the space $\mathscr{C}^{b}\left(\mathbb{D}^{2}\right)$ of uniformly bounded continuous functions on $\mathbb{D}^{2}$ as follows:

$$
\langle T \wedge[\mathfrak{C}], \phi\rangle=\int_{\alpha \in \mathbb{T}} \sum_{j \in J_{\alpha}} h_{\alpha}\left(\xi_{\alpha, j}\right) \phi\left(\xi_{\alpha, j}\right) d \nu(\alpha), \quad \phi \in \mathscr{C}^{b}\left(\mathbb{D}^{2}\right) .
$$

For every $\alpha \in \mathbb{T}$ and $j \in J_{\alpha}$, write, using (5.1) and (5.6),

$$
\begin{aligned}
\xi_{\alpha, j} & =\psi_{\alpha}\left(\zeta_{\alpha, j}\right), \quad \zeta_{\alpha, j}=u_{\alpha, j}+i v_{\alpha, j}, \\
U_{\alpha, j}+i V_{\alpha, j} & :=\left(u_{\alpha, j}+i v_{\alpha, j}\right)^{\gamma} .
\end{aligned}
$$

Recall from Section 5 that the harmonic function $\tilde{h}_{\alpha}(\zeta):=h_{\alpha}\left(\psi_{\alpha}(\zeta)\right)$ is defined on $S_{\lambda}$ and that the harmonic function $\tilde{H}_{\alpha}:=\tilde{h}_{\alpha} \circ \phi^{-1}$ is defined in the upper half plane $\{U+i V: V>0\}$. Applying the Poisson representation formula the upper half plane yields that

$$
h_{\alpha}\left(\xi_{\alpha, j}\right)=\tilde{h}_{\alpha}\left(\zeta_{\alpha, j}\right)=\tilde{H}_{\alpha}\left(U_{\alpha, j}+i V_{\alpha, j}\right)=\frac{1}{\pi} \int_{-\infty}^{\infty} \tilde{H}_{\alpha}(y) \frac{V_{\alpha, j}}{V_{\alpha, j}^{2}+\left(y-U_{\alpha, j}\right)^{2}} d y .
$$

For $\nu$-almost every $\alpha \in \mathbb{T}$, write

$$
\|T \wedge[\mathfrak{C}]\|_{\alpha}:=\frac{1}{\pi} \int_{-\infty}^{\infty} \tilde{H}_{\alpha}(y) \sum_{j \in J_{\alpha}} \frac{V_{\alpha, j}}{V_{\alpha, j}^{2}+\left(y-U_{\alpha, j}\right)^{2}} d y .
$$

We obtain the following formula

$$
\|T \wedge[\mathfrak{C}]\|=\int_{\alpha \in \mathbb{T}}\|T \wedge[\mathfrak{C}]\|_{\alpha} d \nu(\alpha) .
$$

Recall from (5.5) the sector $S_{\lambda}$ in the upper-half plane. 
Proposition 7.7. Let $c, \rho>1$ and $m>0$ be three constants. For $\nu$-almost every $\alpha \in \mathbb{T}$ assume that

- there is a $\mathscr{C}^{1}$-map $\chi^{\alpha}: D^{\alpha} \rightarrow S_{\lambda}$ be defined on a closed interval $D^{\alpha} \subset \mathbb{R}$ such that $c^{-1} \leq\left|\left(\chi^{\alpha}\right)^{\prime}(t)\right| \leq c$;

- there is a sequence of points $\left(t_{\alpha, j}\right)_{j \in J_{\alpha}} \subset D^{\alpha}$ such that the intervals $\left[t_{\alpha, j}-\right.$ $\left.\rho^{-1} m, t_{\alpha, j}+\rho^{-1} m\right]$ for $j \in J_{\alpha}$ are pairwise disjoint and that

$$
\bigcup_{j \in J_{\alpha}}\left[t_{\alpha, j}-\rho^{-1} m, t_{\alpha, j}+\rho^{-1} m\right] \subset D^{\alpha} \subset \bigcup_{j \in J_{\alpha}}\left[t_{\alpha, j}-\rho m, t_{\alpha, j}+\rho m\right] .
$$

Write, using (5.1) and (5.6), for $t \in D^{\alpha}$,

$$
\begin{aligned}
\xi_{\alpha}(t) & =\psi_{\alpha}\left(\chi^{\alpha}(t)\right), \quad \chi^{\alpha}(t)=u_{\alpha}(t)+i v_{\alpha}(t), \\
U_{\alpha}(t)+i V_{\alpha}(t) & :=\left(u_{\alpha}(t)+i v_{\alpha}(t)\right)^{\gamma} .
\end{aligned}
$$

This is the continuous version of (7.6). Consider the function $K^{\alpha}: \mathbb{R} \rightarrow \mathbb{R}^{+}$and the real number $\kappa \in \mathbb{R}^{+}$defined by

$$
\begin{aligned}
K^{\alpha}(y) & :=\frac{1}{m} \int_{D^{\alpha}} \frac{V_{\alpha}(t)}{V_{\alpha}(t)^{2}+\left(y-U_{\alpha}(t)\right)^{2}}, \quad y \in \mathbb{R} ; \\
\kappa & :=\frac{1}{\pi} \int_{-\infty}^{\infty} \tilde{H}_{\alpha}(y) K^{\alpha}(y) d y .
\end{aligned}
$$

1) If, moreover, $c^{-1} h_{\alpha}\left(\xi_{\alpha, j}\right) \leq h_{\alpha}\left(\xi_{\alpha}(t)\right) \leq c h_{\alpha}\left(\xi_{\alpha, j}\right)$ for all $j \in J_{\alpha}, t \in$ $\left[t_{\alpha, j}-\rho m, t_{\alpha, j}+\rho m\right]$, then

$$
c^{-2} \rho^{-1} \kappa \leq\|T \wedge[\mathfrak{C}]\| \leq c^{2} \rho \kappa .
$$

2) If, moreover,

$$
c^{-1} \frac{V_{\alpha, j}}{V_{\alpha, j}^{2}+\left(y-U_{\alpha, j}\right)^{2}} \leq \frac{V_{\alpha}(t)}{V_{\alpha}(t)^{2}+\left(y-U_{\alpha}(t)\right)^{2}} \leq c \frac{V_{\alpha, j}}{V_{\alpha, j}^{2}+\left(y-U_{\alpha, j}\right)^{2}}
$$

for all $j \in J_{\alpha}, t \in\left[t_{\alpha, j}-\rho m, t_{\alpha, j}+\rho m\right]$ and $y \in \mathbb{R}$, then

$$
c^{-2} \rho^{-1} K^{\alpha}(y) \leq \sum_{j \in J_{\alpha}} \frac{V_{\alpha, j}}{V_{\alpha, j}^{2}+\left(y-U_{\alpha, j}\right)^{2}} \leq c^{2} \rho K^{\alpha}(y), \quad y \in \mathbb{R} .
$$

In particular, the concluding estimate of Part 1) holds.

Proof. The idea is to approximate a Riemann sum of a function by its integral. The proof follows easily from Proposition 7.6 and (7.6)-(7.9) .

Definition 7.8. If the assumption of Part 1) of Proposition 7.7 holds, then we say that $\left(K^{\alpha}\right)_{\alpha \in \mathbb{T}}$ given by (7.11) is an interpretation of the geometric intersection $T \wedge[\mathfrak{C}]$ on $\mathbb{U}$ with parametrization $\left(\chi^{\alpha}\right)_{\alpha \in \mathbb{T}}$ and with size $(c, \rho, m)$. Moreover, $m$ is said to be the mesh of the interpretation.

If the assumption of Part 2) of Proposition 7.7holds, then we say that $\left(K^{\alpha}\right)_{\alpha \in \mathbb{T}}$ is a coherent interpretation of the geometric intersection $T \wedge[\mathfrak{C}]$ on $\mathbb{U}$. 
The following result studies the behavior of the Poisson kernel $\frac{V}{V^{2}+(y-U)^{2}}$ in terms of $u$ and $v$.

Lemma 7.9. (Nguyên [27, Lemma 3.3]) There are constants $c_{1}, c_{2}, c_{3}>1$ large enough with $c_{3}>c_{2}$ such that the following properties hold for all $(u, v) \in \mathbb{R}^{2}$ with $\min \{v, b u+a v\} \geq 1$.

1)

$\frac{1}{c_{1}} \leq \frac{(\max \{v, b u+a v\})^{\gamma}}{\sqrt{V^{2}+U^{2}}} \leq c_{1}$ and $\frac{1}{c_{1}} \leq \frac{(\max \{v, b u+a v\})^{\gamma-1} \min \{v, b u+a v\}}{V} \leq c_{1}$.

2) If $\max \{v, b u+a v\} \geq c_{2}(1+|y|)^{1 / \gamma}$, then

$$
\frac{1}{c_{1}} \frac{\min \{v, b u+a v\}}{(\max \{v, b u+a v\})^{\gamma+1}} \leq \frac{V}{V^{2}+(y-U)^{2}} \leq c_{1} \frac{\min \{v, b u+a v\}}{(\max \{v, b u+a v\})^{\gamma+1}} .
$$

3) If $\max \{v, b u+a v\} \leq c_{2}^{-1}(1+|y|)^{1 / \gamma}$, then

$$
\frac{1}{c_{1}} \frac{V}{(1+|y|)^{2}} \leq \frac{V}{V^{2}+(y-U)^{2}} \leq c_{1} \frac{V}{(1+|y|)^{2}} .
$$

4) If $c_{2}^{-1}(1+|y|)^{1 / \gamma} \leq v, b u+a v \leq c_{2}(1+|y|)^{1 / \gamma}$, then

$$
\frac{1}{c_{1}} \frac{1}{(1+|y|)} \leq \frac{V}{V^{2}+(y-U)^{2}} \leq c_{1} \frac{1}{(1+|y|)} .
$$

5) If $\min \{v, b u+a v\} \leq c_{3}^{-1}(1+|y|)^{1 / \gamma}$ and $c_{2}^{-1}(1+|y|)^{1 / \gamma} \leq \max \{v, b u+a v\} \leq$ $c_{2}(1+|y|)^{1 / \gamma}$, then

$$
\frac{1}{c_{1}} \leq \frac{V}{V^{2}+(y-U)^{2}}: \frac{(1+|y|)^{1 / \gamma-1} \min \{v, b u+a v\}}{(\min \{v, b u+a v\})^{2}+(\max \{v, b u+a v\}-\rho)^{2}} \leq c_{1},
$$

where $\rho$ is a real number which depends only on $y$ and on $t:=\min \{v, b u+a v\}$ which satisfies $c_{2}^{-1}(1+|y|)^{1 / \gamma} \leq \rho \leq c_{2}(1+|y|)^{1 / \gamma}$.

In fact, $\rho(y, t)$ is defined as follows. When $c_{3}>1$ is large enough, then for every $1 \leq t \leq c_{3}^{-1}(1+|y|)^{1 / \gamma}$, there exists a solution $u:=u(y, t), v:=v(y, t)$ of the following equation

$$
U=y, \quad \text { where } U+i V=(u+i v)^{\gamma}
$$

which satisfies $c_{2}^{-1}(1+|y|)^{1 / \gamma} \leq \max \{v(y, t), b u(y, t)+a v(y, t)\} \leq c_{2}(1+|y|)^{1 / \gamma}$. So we define

$$
\rho(y, t):=b u(y, t)+a v(y, t) .
$$

8. Test CuRVES $\mathfrak{C}_{r}, \mathfrak{C}_{r, N} \ldots$ AND SECOND REDUCTION

We first introduce some families of algebraic curves on $X$ and a family of analytic curves on an open neighborhood of a given singular point of $\mathscr{F}$. Next, we state basic estimates and deduce the main estimate from the former ones. The proof of the basic estimates will be developed in subsequent sections. 
Since $X$ is projective, we may find a finite family of surjective holomorphic maps $\Psi_{j}: X \rightarrow \mathbb{P}^{2}, 1 \leq j \leq s$, such that for every point $x \in X$, there is at least one map $\Psi_{j}$ which is locally biholomorphic at $x$. Indeed, it suffices to embed $X$ into $\mathbb{P}^{N}$ with $N$ large enough, and choose a family of central projections from $X$ onto $\mathbb{P}^{2}$.

Now fix $x_{0} \in E$ and assume that $\Psi:=\Psi_{j_{0}}: X \rightarrow \mathbb{P}^{2}$ is locally biholomorphic at $x_{0}$. Moreover, suppose without loss of generality that $\Psi$ maps an open neighborhood $\mathbb{V}$ of $x_{0}$ biholomorphically onto the bidisc $\mathbb{D}^{2} \hookrightarrow \mathbb{P}^{2}$ and that $\Psi\left(x_{0}\right)=0$ with $0:=(0,0) \in \mathbb{C}^{2}$. Let $(Z, W)$ be the canonical coordinates of the canonical injection $\mathbb{C}^{2} \hookrightarrow \mathbb{P}^{2}$, i.e. $\mathbb{C}^{2} \simeq\left\{[Z: W: 1]:(Z, W) \in \mathbb{C}^{2}\right\} \subset \mathbb{P}^{2}$.

Since $x_{0}$ is a hyperbolic singular point, we may assume without loss of generality that there are holomorphic coordinates $(z, w)$ defined on $\mathbb{D}^{2}$ such that $(z(0), w(0))=(0,0)=0$ and that the the foliation $\left(\left.\Psi\right|_{\mathbb{V}}\right)_{*} \mathscr{F}$ is associated with the vector field

$$
F(z, w)=z \frac{\partial}{\partial z}+\lambda w \frac{\partial}{\partial w} \quad \text { with some complex number } \quad \lambda=a+i b, \quad b \neq 0 .
$$

So two analytic curves $\{z=0\}$ and $\{w=0\}$ describe two separatrices $\left(\left.\Psi\right|_{\mathbb{V}}\right)_{*} \mathscr{F}$ at 0 . By performing a linear change of coordinates, we may suppose without loss of generality that the complex line $\{Z=0\}$ (resp. $\{W=0\}$ ) is tangent to the separatrice $\{z=0\}$ (resp. $\{w=0\}$ ) at 0 . By dilating the coordinates $(Z, W)$ if necessary, we may assume without loss of generality that the Jacobian matrix of $(Z, W)$ over $(z, w)$ at $(0,0)$ is the identity matrix, i.e.,

$$
\left(\begin{array}{cc}
\frac{\partial Z}{\partial z}(0,0) & \frac{\partial Z}{\partial W}(0,0) \\
\frac{\partial W}{\partial z}(0,0) & \frac{\partial W}{\partial w}(0,0)
\end{array}\right)=\left(\begin{array}{ll}
1 & 0 \\
0 & 1
\end{array}\right) .
$$

In this work we use both systems of coordinates $(Z, W)$ and $(z, w)$. Each system has its own advantages and drawbacks. Indeed, the coordinates $(Z, W)$ appears to be very useful in our cohomological argument, but this argument is only of global nature. On the opposite side, although the coordinates $(z, w)$ are not appropriate for a global argument as the cohomological one, they seem to be very convenient for doing a local analysis near singular points.

Recall that $\Psi$ maps $\mathbb{V}$ biholomorphically onto $\mathbb{D}^{2}$. By shrinking $\mathbb{D}^{2}$ if necessary, the holomorphic implicit function theorem, applied to $\{z=0\}$, allows us to write for $(Z, W) \in \mathbb{D}^{2}$,

$$
z=\theta(Z, W) z_{\infty}(Z, W),
$$

where $\theta(Z, W), z_{\infty}(Z, W)$ are holomorphic functions on $\mathbb{D}^{2}$ with

$$
z_{\infty}(Z, W)=Z-\sum_{j=2}^{\infty} a_{j} W^{j}, \quad a_{j} \in \mathbb{C},
$$

and

$$
1 / 2<|\theta(Z, W)|<2 \quad \text { on } \quad \mathbb{D}^{2} \text { and } \theta(0,0)=1 \text {. }
$$


- Analytic curves $\mathfrak{C}_{r}$ : For every $r \geq 0$ small enough, let $\mathfrak{C}_{r}$ be the complex analytic curve in $\mathbb{V}$ given by

$$
\{x \in \mathbb{V}: z(\Psi(x))=r\} .
$$

Clearly, $\mathfrak{C}_{r} \cap E=\varnothing$ for $r \neq 0$.

- Analytic curves $\mathfrak{C}_{N}^{d}$ : For every $N \in \mathbb{N}$ with $N>2$ and $d \in \mathbb{C} \backslash\{0\}$, let $\mathfrak{C}_{N}^{d}$ be the complex analytic curve in $\mathbb{V}$ given by

$$
\left\{x \in \mathbb{V}: z(\Psi(x))=d(w(\Psi(x)))^{N}\right\} .
$$

Clearly, $\mathfrak{C}_{N}^{d} \cap E=\left\{x_{0}\right\}$.

- Algebraic curves $\mathfrak{C}_{r, N}^{\prime}, \mathfrak{C}_{r, N}$ : Given $r \geq 0$ and $N \in \mathbb{N}$ with $N>2$, define $\mathfrak{C}_{r, N}^{\prime}$ to be the algebraic curve in $\mathbb{P}^{2}$ which is the closure in $\mathbb{P}^{2}$ of the following affine curve

$$
\left\{(Z, W) \in \mathbb{C}^{2}: z_{N}(Z, W)=r\right\} \subset \mathbb{C}^{2},
$$

where $z_{N}(Z, W)$ is the Taylor expansion of order $N$ of $z(Z, W)$, i.e.,

$$
z_{N}(Z, W):=Z-\sum_{j=2}^{N-1} a_{j} W^{j}, \quad(Z, W) \in \mathbb{D}^{2} .
$$

Let $\mathfrak{C}_{r, N}$ be the algebraic curve in $X$ given by

$$
\mathfrak{C}_{r, N}:=\left(\left.\Psi\right|_{\mathbb{V}}\right)^{*}\left(\mathfrak{C}_{r, N}^{\prime \prime}\right)
$$

Basic geometric properties of these algebraic curves are collected in the following.

Proposition 8.1. For every $N \in \mathbb{N}$ with $N>2$, there exists $0 \leq r_{N}<1 / 2$ such that

(i) $\mathfrak{C}_{0, N} \cap E=\left\{x_{0}\right\}$;

(ii) $\mathfrak{C}_{r, N} \cap E=\varnothing$ for every $0<r \leq r_{N}$;

(iii) $\left[\mathfrak{C}_{r, N}\right]$ is cohomologous to $\left[\mathfrak{C}_{0, N}\right]$ in $X$ for every $0 \leq r \leq r_{N}$.

Proof. First, recall the equation $\mathfrak{C}_{r, N}:=\left(\left.\Psi\right|_{\mathbb{V}}\right)^{*}\left(\mathfrak{C}_{r, N}^{\prime}\right)$. Consequently, observe that $x_{0} \in \mathfrak{C}_{0, N}$ as $0 \in \mathfrak{C}_{0, N}^{\prime}$ and that $x_{0} \notin \mathfrak{C}_{r, N}$ for $r>0$ as $0 \notin \mathfrak{C}_{r, N}^{\prime}$ for $r \neq 0$. This discussion, combined with the fact that $E$ is a finite set, implies that for $r_{N}>0$ small enough, both properties (i) and (ii) are satisfied. Finally, property (iii) follows from (8.8) and the fact that two algebraic curves $\mathfrak{C}_{r, N}^{\prime \prime}$ and $\mathfrak{C}_{0, N}^{\prime \prime}$ of the same degree $N$ are cohomologous in $\mathbb{P}^{2}$.

Let $\rho_{a}:=\left(\lim \sup _{j \rightarrow \infty}\left|a_{j}\right|^{1 / j}\right)^{-1} \in(0, \infty]$. So $\rho_{a}$ is the radius of convergence of the analytic function $z_{N}(Z, W)$ defined in (8.7). Clearly, $\rho_{a} \neq \infty$, otherwise the non-constant holomorphic map $\mathbb{C} \ni W \mapsto\left(\sum_{j=2}^{\infty} a_{j} W^{j}, W\right) \subset\{z=0\}$ contradicts our assumption.

Remark 8.2. Together with Lemma 2.4, this is the place where the Brody hyperbolic assumption has fully been used (see also Remark 2.2). 
For the sake of clarity, we may assume without loss of generality that $\rho_{a}=1$. In the sequel we fix a sequence $N_{j} \nearrow \infty$ such that

$$
\lim _{j \rightarrow \infty}\left|a_{N_{j}}\right|^{1 / N_{j}}=\limsup _{j \rightarrow \infty}\left|a_{j}\right|^{1 / j}=\rho_{a}^{-1}=1,
$$

and we always choose $N=N_{j}$ for some $j$ large enough.

For $r>0$, recall that $\mathbb{B}_{r}$ denotes the ball centered at 0 with radius $r$ in $\mathbb{D}^{2} \hookrightarrow X$. The basic estimates which are the main ingredients for the proof of Theorem 6.2 are stated in the following four propositions. Their proofs will be established in the subsequent four sections.

Proposition 8.3. For every $0<\delta<1$, there is $c_{\delta}>1$ such that for every harmonic current $T$,

$$
c_{\delta}^{-1} G\left(x_{0}, r\right) \leq\left\|T \wedge\left[\mathfrak{C}_{r}\right]\right\|_{\mathbb{B}_{r} \delta} \leq c_{\delta} G\left(x_{0}, r\right), \quad 0<r<1 / 2 .
$$

Here $G\left(x_{0}, r\right)$ is defined by (6.1).

Proposition 8.4. For every $N$ large enough in the sequence $\left(N_{j}\right)_{j=1}^{\infty}$ given in (8.9), there is a constant $c=c_{N}>1$ such that for every harmonic current $T$,

$$
\left\|T \wedge\left[\mathfrak{C}_{0, N}\right]\right\|_{\mathbb{B}_{r}} \leq c G\left(x_{0}, r\right), \quad 0<r<1 / 2 .
$$

Proposition 8.5. For every $N$ large enough in the sequence $\left(N_{j}\right)_{j=1}^{\infty}$ given in (8.9), there are constants $c=c_{N}>1$ and $0<r_{N}<1 / 2$ such that for every $0<r<r_{N}$,

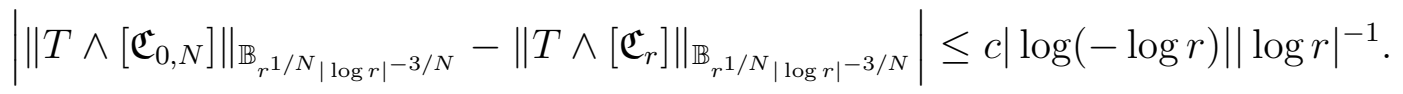

Proposition 8.6. Let $N=N_{j}$ be large enough in the sequence $\left(N_{j}\right)_{j=1}^{\infty}$ given in (8.9). Then the geometric intersection $T \wedge\left[\mathfrak{C}_{r}\right]$ (resp. $T \wedge\left[\mathfrak{C}_{0, N}\right]$ ) on $\mathbb{B}_{r^{1 / N}|\log r|^{-3 / N}}$ admits a coherent interpretation $\left(K^{\alpha}\right)_{\alpha \in \mathbb{T}}$ of the form $K^{\alpha}:=K_{-\log r, N}$ (resp. a coherent interpretation $\left(K^{* \alpha}\right)_{\alpha \in \mathbb{T}}$ of the form $\left.K^{* \alpha}:=K_{-\log r, N}^{*}\right)$. Here

$$
\mathbb{R} \ni y \mapsto K_{-\log r, N}(y) \quad \text { and } \quad \mathbb{R} \ni y \mapsto K_{-\log r, N}^{*}(y)
$$

are functions such that there are constants $c, \kappa>1$ independent of $N$ and a constant $c_{N}>1$ with the following properties:

(i) for $(1+|y|)^{1 / \gamma} \leq \kappa^{-1} s$, we have $K_{s, N}^{*}(y) \leq c s^{1-\gamma}$ and

$$
c^{-1} \leq \frac{K_{s, N}(y)}{N^{\gamma-1} s^{1-\gamma}} \leq c ;
$$

(ii) for $(1+|y|)^{1 / \gamma} \geq \kappa s$, we have

$$
c^{-1} \leq \frac{K_{s, N}^{*}(y)}{N(1+|y|)^{1 / \gamma-1}} \leq c \quad \text { and } \quad c^{-1} \leq \frac{K_{s, N}(y)}{(1+|y|)^{1 / \gamma-1}} \leq c ;
$$

(iii) for $\kappa^{-1} s \leq(1+|y|)^{1 / \gamma} \leq \kappa s$, we have

$$
c_{N}^{-1} \leq \frac{K_{s, N}^{*}(y)}{(1+|y|)^{1 / \gamma-1}} \leq c_{N} \quad \text { and } \quad c_{N}^{-1} \leq \frac{K_{s, N}(y)}{(1+|y|)^{1 / \gamma-1}} \leq c_{N} .
$$


Now we are in the position to reduce the proof of Theorem 6.2 to those of Propositions 8.5 and 8.6 modulo Propositions 8.3 , 8.4. This is the second reduction.

End of the proof of Theorem 6.2, Let $N \geq 1$ be large enough. By Proposition 8.5, there are constants $c_{N}$ and $r_{N}$ such that for every $0<r<r_{N}$,

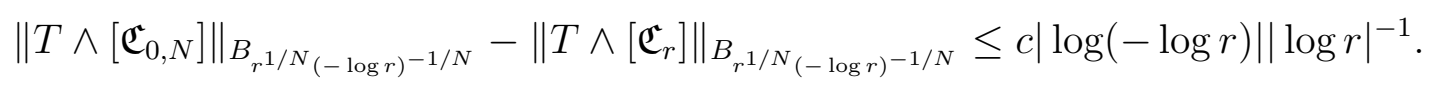

By Proposition 8.6, the geometric intersections $T \wedge\left[\mathfrak{C}_{r}\right]$ and $T \wedge\left[\mathfrak{C}_{0, N}\right]$ on $\mathbb{B}_{r^{1 / N}|\log r|^{-3 / N}}$ admit coherent interpretations $K_{-\log r, N}$ and $K_{-\log r, N}^{*}$ respectively. Consequently, there are two functions $\vartheta, \vartheta^{*}: \mathbb{R} \rightarrow\left[c^{\prime-1}, c^{\prime}\right]$ for some constant $c^{\prime}>1$ such that the above inequality can be rewritten as follows:

$$
\int_{\alpha \in \mathbb{T}}\left(\int_{-\infty}^{\infty}\left(\vartheta^{*}(y) K_{-\log r, N}^{*}(y)-\vartheta(y) K_{-\log r, N}(y)\right) \tilde{H}_{\alpha}(y) d y\right) d \nu(\alpha) \leq c|\log (-\log r)||\log r|^{-1} .
$$

This implies that

$$
I_{2} \leq I_{1}+I_{3}+c|\log (-\log r)||\log r|^{-1},
$$

where

$$
I_{k}:=\left|\int_{\alpha \in \mathbb{T}}\left(\int_{D_{k}}\left(\vartheta^{*}(y) K_{-\log r, N}^{*}(y)-\vartheta(y) K_{-\log r, N}(y)\right) \tilde{H}_{\alpha}(y) d y\right) d \nu(\alpha)\right|,
$$

with $D_{1}:=\left\{y \in \mathbb{R}: \kappa(1+|y|)^{1 / \gamma} \leq-\log r\right\}$, and $D_{2}:=\left\{y \in \mathbb{R}:(1+|y|)^{1 / \gamma} \geq\right.$ $-\kappa \log r\}$, and $D_{3}:=\left\{y \in \mathbb{R}: \kappa^{-1}(1+|y|)^{1 / \gamma} \leq-\log r \leq \kappa(1+|y|)^{1 / \gamma}\right\}$.

Now we apply Proposition 8.6 (i)-(ii)-(iii) to $I_{1}, I_{2}$ and $I_{3}$ respectively. Let $N$ be large enough in the sequence (8.9) which also satisfies $N^{\min \{1, \gamma-1\}} \geq 2 c^{2}$. So we have that

$$
\begin{aligned}
& I_{1} \leq\left(c^{-1} N^{\gamma-1}-c\right) \int_{\alpha \in \mathbb{T}}\left(\int_{D_{1}}(-\log r)^{1-\gamma} \tilde{H}_{\alpha}(y) d y\right) d \nu(\alpha), \\
& I_{2} \geq\left(c^{-1} N-c\right) \int_{\alpha \in \mathbb{T}}\left(\int_{D_{2}}(1+|y|)^{1 / \gamma-1} \tilde{H}_{\alpha}(y) d y\right) d \nu(\alpha), \\
& I_{3} \leq\left(c^{\prime} c_{N}-c^{\prime-1} c_{N}^{-1}\right) \int_{\alpha \in \mathbb{T}}\left(\int_{D_{3}}(1+|y|)^{1 / \gamma-1} \tilde{H}_{\alpha}(y) d y\right) d \nu(\alpha) .
\end{aligned}
$$

This, combined with (8.10) and (6.3), implies that

$$
\begin{aligned}
& \int_{\alpha \in \mathbb{T}}\left(\int_{D_{2}} K_{-\log r}(y) \tilde{H}_{\alpha}(y) d y\right) d \nu(\alpha) \lesssim|\log (-\log r)||\log r|^{-1} \\
+ & \int_{\alpha \in \mathbb{T}}\left(\int_{D_{1}} K_{-\log r}(y) \tilde{H}_{\alpha}(y) d y\right) d \nu(\alpha)+\int_{\alpha \in \mathbb{T}}\left(\int_{D_{3}} K_{-\log r}(y) \tilde{H}_{\alpha}(y) d y\right) d \nu(\alpha) .
\end{aligned}
$$


Hence,

$$
\begin{aligned}
\int_{\alpha \in \mathbb{T}}\left(\int_{D_{1} \cup D_{2} \cup D_{3}} K_{-\log r}(y) \tilde{H}_{\alpha}(y) d y\right) d \nu(\alpha) \lesssim|\log (-\log r)||\log r|^{-1} \\
\quad+\int_{\alpha \in \mathbb{T}}\left(\int_{D_{1} \cup D_{3}} K_{-\log r}(y) \tilde{H}_{\alpha}(y) d y\right) d \nu(\alpha) .
\end{aligned}
$$

Since we know by Lemma 6.1 that the left-hand side of the last line is equivalent to $G(r)$, the desired conclusion of the theorem follows when the constant $c_{0}$ is large enough.

\section{COHOMOLOGICAL RELATION AND THIRD REDUCTION}

We first state several basic estimates. Next, using these estimate we establish a cohomological invariance result (see Proposition 9.3). Finally, we deduce from this result Proposition 8.5. Consequently, modulo Propositions [8.3, 8.4, 9.1, 9.2, the proof of Theorem 6.2 is reduced to that of Proposition 8.6. This is the last reduction.

Proposition 9.1. For every $N$ large enough in the sequence $\left(N_{j}\right)_{j=1}^{\infty}$ given in (8.9), there exist constants $c=c_{N}>1, \delta=\delta_{N}>0$ and a constant $r_{N}$ satisfying the conclusion of Proposition 8.1 with the following properties. For every $0<r<r_{N}$ and for every harmonic current $T$ tangent to $\mathscr{F}$ of mass 1 , the following mass estimates hold:

$$
\begin{aligned}
& \left|\left\|T \wedge\left[\mathfrak{C}_{r, N}\right]\right\|_{X \backslash \mathbb{D}^{2}}-\left\|T \wedge\left[\mathfrak{C}_{0, N}\right]\right\|_{X \backslash \mathbb{D}^{2}}\right| \leq c r^{\delta} \\
& \left|\left\|T \wedge\left[\mathfrak{C}_{r, N}\right]\right\|_{\mathbb{D}^{2} \backslash \mathbb{B}_{r^{1 / N}|\log r|^{3 / N}}}-\left\|T \wedge\left[\mathfrak{C}_{0, N}\right]\right\|_{\mathbb{D}^{2} \backslash \mathbb{B}_{r^{1 / N}|\log r|^{3 / N}}}\right| \leq c|\log r|^{-1},
\end{aligned}
$$

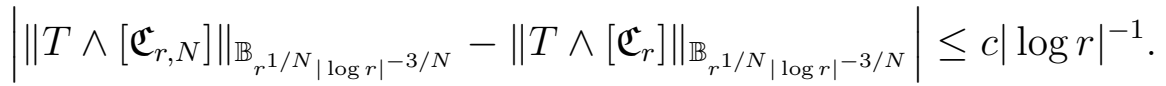

We postpone the proof of Proposition 9.1 to Section 12.

For $0<r<1 / 2$ and $N \geq 2$, consider the corona

$$
\mathbb{A}_{r, N}:=\mathbb{B}_{r^{1 / N}|\log r|^{3 / N}} \backslash \mathbb{B}_{r^{1 / N}|\log r|^{-3 / N}}
$$

So we obtain the following partition of $X$ :

$$
X=\left(X \backslash \mathbb{D}^{2}\right) \coprod\left(\mathbb{D}^{2} \backslash \mathbb{B}_{r^{1 / N}|\log r|^{3 / N}}\right) \coprod \mathbb{A}_{r, N} \coprod \mathbb{B}_{r^{1 / N}|\log r|^{-3 / N}}
$$

Proposition 9.2. For every $N$ large enough in the sequence $\left(N_{j}\right)_{j=1}^{\infty}$ given in (8.9), there are constants $0<r_{N} \ll 1$ and $c=c_{N}>1$ such that for every $0<r<r_{N}$,

$$
\begin{aligned}
\| T & \wedge\left[\mathfrak{C}_{r}\right] \|_{\mathbb{A}_{r, N}} \leq c|\log (-\log r)||\log r|^{-1}, \\
\| T & \wedge\left[\mathfrak{C}_{0, N}\right] \|_{\mathbb{A}_{r, N}} \leq c|\log (-\log r)||\log r|^{-1}, \\
\| T & \wedge\left[\mathfrak{C}_{r, N}\right] \|_{\mathbb{A}_{r, N}} \leq c|\log (-\log r)||\log r|^{-1} .
\end{aligned}
$$


The proof of Propostion 9.2 will occupy Section 13 ,

Taking for granted these estimates, we want to prove the following cohomological invariance result.

Proposition 9.3. Let $r_{N}$ be given by Proposition 8.1 for every $N \in \mathbb{N}$ with $N>2$. Then for every $0<r<r_{N}$ and for every harmonic current $T$, we have that $\left\|\left[\mathfrak{C}_{r, N}^{\mathfrak{*}}\right] \wedge T\right\|_{X}=\left\|\left[\mathfrak{C}_{0, N}\right] \wedge T\right\|_{X}$.

This result does not follow from Proposition 7.4 since $\mathfrak{C}_{0, N} \cap E \neq \varnothing$. We need the following auxiliary result.

Lemma 9.4. We have $\lim _{r \rightarrow 0+}\left\|T \wedge\left[\mathfrak{C}_{r, N}\right]\right\|_{X}=\left\|T \wedge\left[\mathfrak{C}_{0, N}\right]\right\|_{X}$.

Proof. Combining estimates (9.1)-(9.2), we get that

$$
\begin{aligned}
\mid\left\|T \wedge\left[\mathfrak{C}_{r, N}\right]\right\|_{X}-\| T \wedge & {\left[\mathfrak{C}_{0, N}\right] \|\left._{X}|\leq c| \log r\right|^{-1} } \\
& +\left\|T \wedge\left[\mathfrak{C}_{r, N}\right]\right\|_{\mathbb{B}_{r^{1 / N|\log r|}} / N}+\left\|T \wedge\left[\mathfrak{C}_{0, N}\right]\right\|_{\mathbb{B}_{r^{1 / N}|\log r|^{3 / N}}} .
\end{aligned}
$$

In the remainder of the proof we will that the two terms in the last line tend to 0 as $r \rightarrow 0+$. This will imply the lemma.

Applying (9.7) yields that

$$
\left\|T \wedge\left[\mathfrak{C}_{r, N}\right]\right\|_{\mathbb{B}_{r^{1 / N}|\log r|^{3 / N}}} \leq c|\log (-\log r)||\log r|^{-1}+\left\|T \wedge\left[\mathfrak{C}_{r}\right]\right\|_{\mathbb{R}_{r^{1 / N}|\log r|^{-3 / N}}} .
$$

Consequently, we infer that

$$
\begin{aligned}
\lim _{r \rightarrow 0+}\left\|T \wedge\left[\mathfrak{C}_{r, N}\right]\right\|_{\mathbb{B}_{r^{1 / N}|\log r|^{3 / N}}} \leq \lim _{r \rightarrow 0+}\left\|T \wedge\left[\mathfrak{C}_{r}\right]\right\|_{\mathbb{B}_{r^{1 / N}|\log r|^{-3 / N}}} \\
\leq \lim _{r \rightarrow 0+}\left\|T \wedge\left[\mathfrak{C}_{r}\right]\right\|_{\mathbb{B}_{r^{1 / 2 N}}=0,}
\end{aligned}
$$

where the last limit holds by Proposition 8.3 applied to $\delta=1 /(2 N)$. Hence, $\lim _{r \rightarrow 0+}\left\|T \wedge\left[\mathfrak{C}_{r, N}\right]\right\|_{\mathbb{B}_{r^{1 / N}|\log r| 3 / N}}=0$.

On the other hand, applying (9.6) yields that

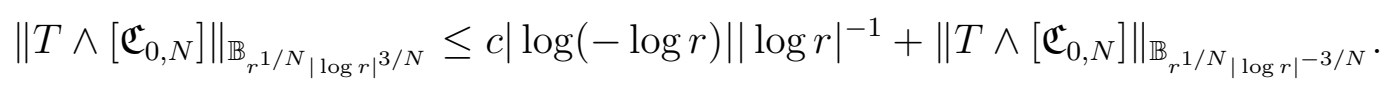

Therefore, we deduce that

$$
\begin{aligned}
\lim _{r \rightarrow 0+}\left\|T \wedge\left[\mathfrak{C}_{0, N}\right]\right\|_{\mathbb{B}_{r^{1 / N}|\log r|} / N} & \leq \lim _{r \rightarrow 0+}\left\|T \wedge\left[\mathfrak{C}_{0, N}\right]\right\|_{\mathbb{B}_{r^{1 / N}|\log r|^{-3 / N}}} \\
& \leq \lim _{r \rightarrow 0+}\left\|T \wedge\left[\mathfrak{C}_{0, N}\right]\right\|_{\mathbb{B}_{r^{1 / N \mid}}}=0,
\end{aligned}
$$

where the last limit holds by Proposition 8.4 applied to $\delta=1 / N$. Hence, $\lim _{r \rightarrow 0+}\left\|T \wedge\left[\mathfrak{C}_{0, N}\right]\right\|_{\mathbb{R}_{r^{1 / N}|\log r|^{3 / N}}}=0$.

End of the proof of Proposition 9.3, By Lemma 9.4, we have that

$$
\left\|T \wedge\left[\mathfrak{C}_{0, N}\right]\right\|_{X}=\lim _{s \rightarrow 0+}\left\|T \wedge\left[\mathfrak{C}_{s, N}\right]\right\|_{X} .
$$


On the other hand, by Proposition 8.1 (ii)-(iii), $\mathfrak{C}_{r, N} \cap E=\varnothing, \mathfrak{C}_{s, N} \cap E=\varnothing$, and both $\left[\mathfrak{C}_{r, N}\right]$ and $\left[\mathfrak{C}_{s, N}\right]$ are cohomologous. Consequently, by Proposition 9.3 , the right hand side of the last limit is equal to $\left\|T \wedge\left[\mathfrak{C}_{r, N}\right]\right\|_{X}$.

End of the proof of Proposition 8.5. Fix $N \in \mathbb{N}$ with $N>2$ and let $r_{N}$ be given by Proposition 9.1 and Proposition 8.1. So by Proposition 9.3, we have, for $0<r<r_{N}$, that

$$
T \wedge\left[\mathfrak{C}_{0, N}\right]=T \wedge\left[\mathfrak{C}_{r, N}\right] .
$$

This, combined with estimates (9.1) $-(9.2)$ and the partition (9.4), implies that

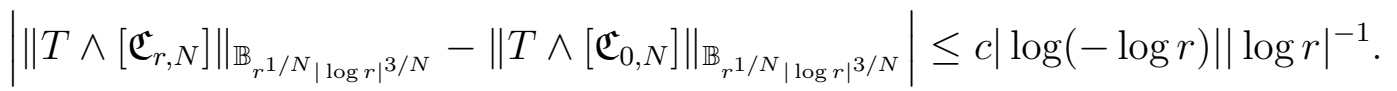

Putting this together with estimates (9.6) and (9.7) yields that

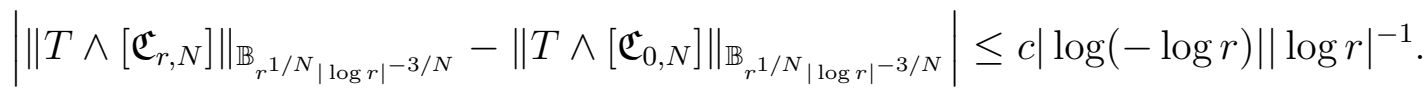

This, coupled with (9.3), gives that

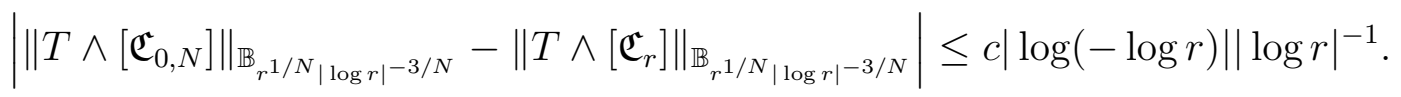

This completes the proof.

\section{INTERSECTION OF TEST CURVES WITH A LEAF}

In Section 8 we introduce the analytic curves $\mathfrak{C}_{r}, \mathfrak{C}_{N}^{d}$ which are defined on a neighborhood of a singular point of the foliation, and the algebraic curves $\mathfrak{C}_{r, N}$ which are defined on the whole $X$. The main purpose of this section is to study the distributions of these test curves with the leaves of the foliations near singularities. Therefore, in what follows, we restrict ourselves to the local model of Section 8 and Section [5, and we keep the notation introduced therein. More specifically, we may assume without loss of generality that $x_{0} \equiv 0 \in$ $\mathbb{D}^{2}$ and that there are holomorphic coordinates $(z, w)$ defined on $\mathbb{D}^{2}$ such that $(z(0), w(0))=(0,0)=0$ and that the the foliation $\left(\left.\Psi\right|_{\mathbb{V}}\right)_{*} \mathscr{F}$ is associated with the vector field $F(z, w)=z \frac{\partial}{\partial z}+\lambda w \frac{\partial}{\partial w}$ with some complex number $\lambda=a+i b, b \neq$ 0 . Note that two analytic curves $\{z=0\}$ and $\{w=0\}$ describe two separatrices $\left(\left.\Psi\right|_{\mathbb{V}}\right)_{*} \mathscr{F}$ at 0 . Recall that $\mathbb{T} \simeq\left\{\alpha \in \mathbb{C}: e^{-2 \pi b} \leq|\alpha| \leq 1\right\}$.

The distribution of the intersection points of $\mathfrak{C}_{r}$ with a leaf in the bidisc $\mathbb{D}^{2}$ is quite simple as the following result shows.

Lemma 10.1. For each $0<r<1$ and each $\alpha \in \mathbb{T}$, the intersection of $\mathfrak{C}_{r}$ with the Riemann surface $\widehat{L}_{\alpha}$ can be parametrized, via (5.1), by

$\xi_{r, \alpha, k}=\psi_{\alpha}\left(\tau_{r, \alpha, k}\right), \quad$ where $\quad \tau_{r, \alpha, k}=u_{\alpha, k}+i v_{r}:=2 k \pi-(\log |\alpha|) / b+i(-\log r), \quad k \in \mathbb{Z}$.

Proof. Let $(z, w)=\psi_{\alpha}(\tau)$ with $\tau=u+i v$ be an intersection point of $\mathfrak{C}_{r}$ with the Riemann surface $\widehat{L}_{\alpha}$. Then $\tau$ is a solution of the equation $r=z=e^{i(\tau+(\log |\alpha|) / b)}$. Solving this equation gives all the solutions (10.1). 
Lemma 10.2. Let $N \in \mathbb{N} \backslash\{0\}$ and $d \in \mathbb{C} \backslash\{0\}$. For each $\alpha \in \mathbb{T}$, the intersection of $\mathfrak{C}_{N}^{d}=\left\{z=d w^{N}\right\}$ with the Riemann surface $\widehat{L}_{\alpha}$ can be parametrized, via (5.1), by

$$
\xi_{N, \alpha, k}=\psi_{\alpha}\left(\tau_{N, \alpha, k}\right), \quad \text { where } \quad \tau_{N, \alpha, k}=u_{N, \alpha, k}+i v_{N, \alpha, k}, \quad k \in \mathbb{N},
$$

and $\left(u_{N, \alpha, k}, v_{N, \alpha, k}\right)$ is the unique solution of the following system of linear equations:

$$
\begin{cases}-(N a-1) u+N b v & =2 \pi k+\arg d+N \arg \alpha+b^{-1}(N a-1) \log |\alpha| \\ N b u+(N a-1) v & =\log |d| .\end{cases}
$$

Moreover, let

$$
t_{N, \alpha, k}:=b u_{N, \alpha, k}+a v_{N, \alpha, k}, \quad k \in \mathbb{N} .
$$

Then there are constants $v_{N}, t_{N}$ such that

$$
v_{N, \alpha, k+1}-v_{N, \alpha, k}=v_{N} \quad \text { and } \quad t_{N, \alpha, k+1}-t_{N, \alpha, k}=t_{N} \quad \text { for } \quad k \in \mathbb{N}
$$

and that

$$
v_{N} \approx N^{-1} \text { and } t_{N} \approx N^{-2}
$$

Proof. Let $(z, w)=\psi_{\alpha}(\tau)$ with $\tau=u+i v$ be an intersection point of $\mathfrak{C}_{N}^{c}$ with the Riemann surface $\widehat{L}_{\alpha}$. Then we deduce from $z=d w^{N}$ that $\tau$ is a solution of the equation

$$
e^{i(\tau+(\log |\alpha|) / b)}=d \alpha^{N} e^{i N \lambda(\tau+(\log |\alpha|) / b)} .
$$

So there is $k \in \mathbb{Z}$ such that

$i(\tau+(\log |\alpha|) / b)=2 i \pi k+\log |d|+i \arg d+N \log |\alpha|+i N \arg \alpha+i N(a+i b)(\tau+(\log |\alpha|) / b)$.

Equating the imaginary and the real parts of both sides, we obtain system (10.3).

Writing $u_{N}:=u_{N, \alpha, k+1}-u_{N, \alpha, k}$, we infer from (10.5) and system (10.3) that $\left(u_{N}, v_{N}\right)$ is a solution of the following system

$$
\begin{cases}-(N a-1) u+N b v & =2 \pi \\ N b u+(N a-1) v & =0 .\end{cases}
$$

So we get that

$$
u_{N}=\frac{-2 \pi(N a-1)}{(N a-1)^{2}+(N b)^{2}}, \quad v_{N}=\frac{2 \pi N b}{(N a-1)^{2}+(N b)^{2}}, \quad t_{N}=\frac{2 \pi b}{(N a-1)^{2}+(N b)^{2}} .
$$

This proves (10.5) and (10.6).

The following result plays a vital role in this section. It allows us to approximate the function $z_{N}$ defined (8.7) efficiently. Consequently, we infer from this result a good picture of the distributions of the intersection of $\mathfrak{C}_{r, N}$ with a general leaf near singularities. 
Proposition 10.3. Let $\mathbb{N} \ni N \mapsto M_{N} \in \mathbb{N}$ be a sequence such that $\lim _{N \rightarrow \infty} M_{N}=$ $\infty$. Then, for every $N \in \mathbb{N}$ large enough in the sequence $\left(N_{j}\right)_{j=1}^{\infty}$ given in (8.9), there is a constant $0<s=r_{N, M}<1$ such that the analytic functions

$z_{\infty}(z, w):=z_{\infty}(Z(z, w), W(z, w)) \quad$ and $\quad z_{N}(z, w):=z_{N}(Z(z, w), W(z, w))$, $z_{\infty}(Z, W)$ (resp. $z_{N}(Z, W)$ ) being the analytic function given in (8.2) (resp. (8.7)), are well-defined on $\mathbb{D}^{2}$ (resp. $\mathbb{D}_{s}^{2}$ ) and that the following two properties hold:

(i) For every $w \in \mathbb{D}_{s}$ and every $0 \leq r \leq|w| / 2$, the equation $z_{N}(z, w)=r$ with $|z| \leq|w|$ admits a unique solution.

(ii) For every point $(z, w) \in \mathbb{D}_{s}^{2}$ with $z_{N}(z, w)=r$ for some $0 \leq r<1$, at least one of the following two items holds:

(ii-a) $|z-r| \leq 4 r^{2}$ and $|w| \leq r$

(ii-b) $\left|z_{N}(z, w)-\left(z_{\infty}(z, w)+a_{N} w^{N}\right)\right| \leq M_{N}^{-1}\left|a_{N}\right||w|^{N}$.

Proof. As in Section 8, we may suppose without loss of generality that the complex line $\{Z=0\}$ (resp. $\{W=0\}$ ) in $\mathbb{P}^{2}$ is tangent to the separatrice $\{z=0\}$ (resp. $\{w=0\}$ ) at 0 . Therefore, arguing as in the proof of $(8.2)$, we obtain the following equation for $W$ :

$$
W=W(z, w)=\vartheta(z, w)\left(w+\sum_{j=2}^{\infty} b_{j} z^{j}\right), \quad b_{j} \in \mathbb{C},
$$

where $\vartheta$ is a holomorphic function on $\mathbb{D}^{2}$ such that

$$
1 / 2<|\vartheta(z, w)|<2 \text { on } \mathbb{D}^{2} \text { and } \vartheta(0,0)=1 .
$$

Let $\rho \in \mathbb{R}^{+}$be such that $\limsup _{j \rightarrow \infty}\left|b_{j}\right|^{1 / j}<\rho$. This together with (8.9) gives an integer $N$ with $N>N_{0}$ and a constant $c>1$ such that

$$
\left|a_{N}\right|>2^{-N}, \quad\left|a_{j}\right|<2^{j} \text { for } j \geq N, \quad\left|b_{k}\right|<c \rho^{k} \text { for } k \geq N .
$$

Inserting (8.2) and (10.7) into (8.7), we get that

$$
z_{N}(z, w)=z_{\infty}(z, w)+\sum_{j=N}^{\infty} a_{j} W^{j}=z_{\infty}(z, w)+\sum_{j=N}^{\infty} a_{j} \vartheta^{j}(z, w)\left(w+\sum_{k=2}^{\infty} b_{k} z^{k}\right)^{j} .
$$

Now we prove assertion (i). We infer from (10.10) and (10.9) and (10.8) that for $s$ small enough and $|z| \leq|w| \leq s$,

$$
\left|z_{N}(z, w)-z_{\infty}(z, w)\right| \leq \sum_{j=N}^{\infty}\left|a_{j}\right||\vartheta(z, w)|^{j}\left(|w|+\sum_{k=2}^{\infty}\left|b_{k}\right||w|^{k}\right)^{j}=O\left(|w|^{2}\right) \ll|w| .
$$

This, combined with $r \leq|w| / 2$, implies that

$$
\left|z_{N}(z, w)-z_{\infty}(z, w)\right|<\left|z_{\infty}(z, w)-r\right| \quad \text { for } \quad z \in \partial \mathbb{D}_{|w|} .
$$

Now let $0<s<1$ be small enough, and fix $w \in \mathbb{D}_{s}$, and fix $0 \leq r<|w| / 2$. Using (8.2)-(8.3) and applying Rouché's theorem to the functions $z \mapsto z-$ 
$r \theta(Z(z, w), W(z, w))$ and $z \mapsto z$ on $\mathbb{D}_{|w|}$, we see easily that the function $z \mapsto$ $z_{\infty}(z, w)-r$ admits a unique solution on $\mathbb{D}_{|w|}$. Next, using (10.11) we apply Rouché's theorem to the functions $z \mapsto z_{N}(z, w)-r$ and $z \mapsto z_{\infty}(z, w)-r$ on $\mathbb{D}_{|w|}$. Consequently, assertion (i) follows.

In the remainder of the proof, we write $M$ instead of $M_{N}$ for the sake of simplicity. To prove assertion (ii) we take for granted the following

Fact. When $s>0$ is small enough and $(z, w) \in\left(\mathbb{D}_{s}\right)^{2}$ with $z_{N}(z, w)=r$ does not satisfies property (ii-a), we have that $|w| \geq 8 M N\left|\sum_{k=2}^{\infty} b_{k} z^{k}\right|$.

Using (10.7) and then the above fact, we see that

$$
\begin{aligned}
\left|\vartheta^{-N}(z, w) W^{N}-w^{N}\right| & \leq \sum_{p=1}^{N}\left(\begin{array}{c}
N \\
p
\end{array}\right)|w|^{N-p}\left|\sum_{k=2}^{\infty} b_{k} z^{k}\right|^{p} \\
& \leq\left(\sum_{p=1}^{N}\left(\begin{array}{c}
N \\
p
\end{array}\right)(8 M N)^{-p}\right)|w|^{N} \\
& \leq\left(\left(1+8^{-1} M^{-1} N^{-1}\right)^{N}-1\right)\left|w^{N}\right| \\
& \leq\left(e^{8^{-1} K^{-1}}-1\right)\left|w^{N}\right| \leq 6^{-1} M^{-1}\left|w^{N}\right| .
\end{aligned}
$$

Moreover, for $s=r_{N, M}>0$ small enough, we infer from (10.7)-(10.8) and the continuity of $\vartheta$ that for $(z, w) \in \mathbb{D}_{s}^{2}$,

$$
\left|\vartheta^{-N}(z, w) W^{N}-W^{N}\right|<12^{-1} M^{-1}\left|W^{N}\right|<4^{-1} M^{-1}\left|w^{N}\right|,
$$

where the last estimate follows from (10.12). On the other hand, using the second inequality in (10.9), (10.7), (10.8) and then the above fact, we see that

$$
\begin{aligned}
\left|\sum_{j=N+1}^{\infty} a_{j} W^{j}\right| & \leq \sum_{j=N+1}^{\infty} 4^{j}\left(|w|+\left|\sum_{k=2}^{\infty} b_{k} z^{k}\right|\right)^{j} \\
& \leq \sum_{j=N+1}^{\infty} 4^{j}\left(1+8^{-1} M^{-1} N^{-1}\right)^{j}|w|^{j} \\
& \leq 2^{-N-1} M^{-1}|w|^{N} \\
& \leq 2^{-1}\left|a_{N} w^{N}\right|,
\end{aligned}
$$

where the third inequality holds when $s=r_{N, M}>0$ is small enough, and the last one follows from the first inequality in (10.9). This, combined with (10.12) 
and (10.13), yields that

$$
\begin{aligned}
& \left|\left(z_{\infty}(z, w)+\sum_{j=N}^{\infty} a_{j} W^{j}\right)-\left(z_{\infty}(z, w)+a_{N} w^{N}\right)\right| \\
\leq & \left|a_{N} \vartheta(z, w)^{-N} W^{N}-a_{N} w^{N}\right|+\left|a_{N} \vartheta(z, w)^{-N} W^{N}-a_{N} W^{N}\right|+\left|\sum_{j=N+1}^{\infty} a_{j} W^{j}\right| \\
\leq & M^{-1}\left|a_{N} w^{N}\right| .
\end{aligned}
$$

Since we know by (8.2) and (8.7) that the left hand side of the last line is equal to $\left|z_{N}(z, w)-\left(z_{\infty}(z, w)+a_{N} w^{N}\right)\right|$, assertion (ii-b) and hence the proposition follow modulo the above fact.

Now we turn to the proof of this fact. Suppose in order to reach a contradiction that

$$
|w| \leq 8 M N\left|\sum_{k=2}^{\infty} b_{k} z^{k}\right| .
$$

This, coupled with (10.10) and (10.7), implies that

$$
\begin{aligned}
\left|z_{\infty}(z, w)-z_{N}(z, w)\right| & \leq \sum_{j=N}^{\infty}\left|a_{j}\right|\left|\vartheta^{j}(z, w)\right|\left(|w|+\left|\sum_{k=2}^{\infty} b_{k} z^{k}\right|\right)^{j} \\
& \leq \sum_{j=N}^{\infty} 4^{j}(1+8 M N)^{j}\left|\sum_{k=2}^{\infty} b_{k} z^{k}\right|^{j} \\
& \leq \sum_{j=N}^{\infty} 4^{j}(1+8 M N)^{j} c^{j}|z|^{2 j}\left(\sum_{k=2}^{\infty} \rho^{k}|z|^{k-2}\right)^{j}
\end{aligned}
$$

where the second inequality holds by the second inequality in (10.9), (10.8) and (10.14), the last one by the third inequality in (10.9). Hence, we infer that for $0<s<1$ small enough,

$$
\left|z_{\infty}(z, w)-z_{N}(z, w)\right| \ll|z|^{2} .
$$

Suppose now that the point $(z, w) \in\left(\mathbb{D}_{s}\right)^{2}$ satisfies the assumption of assertion (ii). We infer from (10.15) that $r=\left|z_{N}(z, w)\right| \geq\left|z_{\infty}(z, w)\right|-|z|^{2}$. Since $s$ is small enough, we infer from (8.2) $-(8.3)$ that $z_{\infty}(z, w) / z$ is close to 1 . So $|z| \leq 2 r$. Hence, (10.15) implies that $\left|z_{\infty}(z, w)-r\right| \leq 4 r^{2}$. Moreover, (10.14), combined with $|z| \leq 2 r$, implies that $|w| \lesssim|z|^{2} \ll r$. Hence, we obtain property (ii-a) which is the desired contradiction. The proof of assertion (ii) is thereby completed.

In what follows by shrinking $\mathbb{D}^{2}$ if necessary, we may assume without loss of generality that the vector field $F$ at the beginning of the section is defined on the bidisc $(e \mathbb{D}) \times\left(e^{|\lambda|} \mathbb{D}\right)$. 
Definition 10.4. Two points $x_{1}=\left(z_{1}, w_{1}\right)$ and $x_{2}=\left(z_{2}, w_{2}\right) \in(\mathbb{D} \backslash\{0\})^{2}$ are said to be quasi-compatible if there is $t \in \mathbb{C}$ such that $z_{2}=z_{1} e^{t}$ and $w_{2}=w_{1} e^{\lambda t}$. Clearly, $x_{1}$ and $x_{2}$ are on the same leaf. If, moreover, we can choose $t$ with $|t|<1$, then we say that $x_{1}$ and $x_{2}$ are compatible.

Given two quasi-compatible points $x_{1}=\left(z_{1}, w_{1}\right)$ and $x_{2}=\left(z_{2}, w_{2}\right) \in \mathbb{D}^{2}$, the compatible pseudo-distance between them, denoted by $\operatorname{dist}_{C}\left(x_{1}, x_{2}\right)$, is defined by

$$
\operatorname{dist}_{C}\left(x_{1}, x_{2}\right):=\max \left\{\frac{\left|z_{1}-z_{2}\right|}{\left|z_{1}\right|}, \frac{\left|z_{1}-z_{2}\right|}{\left|z_{2}\right|}, \frac{\left|w_{1}-w_{2}\right|}{\left|w_{1}\right|}, \frac{\left|w_{1}-w_{2}\right|}{\left|w_{2}\right|}\right\} .
$$

Lemma 10.5. Let $x, x^{\prime} \in(\mathbb{D} \backslash\{0\})^{2}$ be two compatible points. Let $t \in \mathbb{C}$ such that $z_{2}=z_{1} e^{t}$ and $w_{2}=w_{1} e^{\lambda t}$ with $|t|$ smallest possible. Then

(i) $|z| \approx\left|z^{\prime}\right|,|w| \approx\left|w^{\prime}\right|,\|x\| \approx\left\|x^{\prime}\right\|$, and

$$
\operatorname{dist}_{C}\left(x_{1}, x_{2}\right) \approx \frac{\left|z-z^{\prime}\right|}{|z|} \approx \frac{\left|w-w^{\prime}\right|}{|w|} \approx \frac{\left\|x-x^{\prime}\right\|}{\|x\|} \approx|t| ;
$$

(ii) there is a constant $c>1$ such that

$$
c^{-1} \frac{\left\|x-x^{\prime}\right\|}{-\|x\| \log ^{*}\|x\|} \leq \operatorname{dist}_{P}\left(x, x^{\prime}\right) \leq c \frac{\left\|x-x^{\prime}\right\|}{-\|x\| \log ^{*}\|x\|} .
$$

Proof. Assertion (i) is an immediate consequence of Definition 10.4 .

To prove assertion (ii), let $\omega \in \Omega$ be a path such that there is a differentiable function $s \ni[0,1] \mapsto \zeta(s) \in \mathbb{D}$ satisfying

$$
\omega(s):=\left(z e^{\zeta(s)}, w e^{\lambda \zeta(s)}\right) \in\left(e^{1} \mathbb{D}\right) \times\left(e^{|\lambda|} \mathbb{D}\right) \quad \text { for } \quad s \in[0,1] .
$$

and $\zeta(0)=0$ and $\zeta(1)=t$. Hence, $\omega(0)=x$ and $\omega(1)=x^{\prime}$. By Lemma 10.5 (i), we get $\left|\log ^{*}\|\omega(s)\|\right| \approx\left|\log ^{*} \| x\right| \mid$ for $s \in[0,1]$. Therefore, by integrating along the path $[0,1] \ni s \mapsto \zeta(s)$ and applying Part 2) of Lemma 2.4, we get that

$$
\begin{aligned}
\operatorname{dist}_{P}(\omega: 0,1) & =\int_{\omega[0,1]} \sqrt{g_{P}(z)} \\
& =\int_{0}^{1} \zeta^{*}\left(\psi_{x}^{*}\left(\sqrt{g_{P}}\right)\right)=\int_{\zeta[0,1]}\left(\log ^{*}\|x\|\right)^{-1} d s \\
& \gtrsim \frac{|t|}{-\log ^{*} \| x \mid} \approx \frac{\left\|x-x^{\prime}\right\|}{-\|x\| \log ^{*}\|x\|} .
\end{aligned}
$$

When $\zeta(s):=s t$ for $s \in[0,1], \gtrsim$ above becomes $\approx$. This implies assertion (ii).

In the remainder of this section we consider the function

$$
M_{N}:=8^{N}, \quad N \in \mathbb{N} .
$$

By the first inequality in (10.9), this choice ensures that $M_{N}^{-1} \ll\left|a_{N}\right|$. Moreover, we take $N$ so large in the sequence $\left(N_{j}\right)_{j=1}^{\infty}$ given in (8.9) that $N$ and the 
constant $M=M_{N}$ satisfy the conclusion of Proposition 10.3, Let $0<r_{N}:=$ $r_{N, M}<1$ be given by this proposition.

Lemma 10.6. Let $N \in \mathbb{N}$ be as above, let $d:=-a_{N}$, where $a_{N}$ is introduced in (8.7) and $\alpha \in \mathbb{T}$. Let $\xi_{N, \alpha, k}(k \in \mathbb{N})$ be the intersection of $\mathfrak{C}_{N}^{d}=\left\{z=d w^{N}\right\}$ with the Riemann surface $\widehat{L}_{\alpha}$ described by (10.2). Then the intersection of the curve $\mathfrak{C}_{0, N}$ with $\widehat{L}_{\alpha}$ can be enumerated as $\xi_{0, N, \alpha, k}(k \in \mathbb{N})$ such that $\xi_{N, \alpha, k}$ and $\xi_{0, N, \alpha, k}$ are compatible and that

$$
\operatorname{dist}_{C}\left(\xi_{N, \alpha, k}, \xi_{0, N, \alpha, k}\right) \leq c N^{-1} \quad \text { for } \quad k \in \mathbb{N} .
$$

Here $c>1$ is a constant independent of $N, \alpha$ and $k$.

Proof. We need to prove that for every point $\xi_{1} \in \mathfrak{C}_{N}^{d} \cap \widehat{L}_{\alpha}$ (resp. $\xi_{1} \in \mathfrak{C}_{0, N} \cap \widehat{L}_{\alpha}$ ), there is exactly one point $\xi_{2} \in \mathfrak{C}_{0, N} \cap \widehat{L}_{\alpha}$ (resp. $\xi_{2} \in \mathfrak{C}_{N}^{d} \cap \widehat{L}_{\alpha}$ ) such that $\xi_{1}$ and $\xi_{2}$ are compatible and that

$$
\operatorname{dist}_{C}\left(\xi_{1}, \xi_{2}\right) \lesssim N^{-1}
$$

We will only show that for every point $\xi_{1} \in \mathfrak{C}_{N}^{d} \cap \widehat{L}_{\alpha}$, there is exactly one point $\xi_{2} \in \mathfrak{C}_{0, N} \cap \widehat{L}_{\alpha}$ satisfying (10.17) since the other assertion can be proved similarly. Let $s_{0}:=r_{N}$.

Write $\xi_{1}=\left(z_{1}, w_{1}\right)$. So $z_{1}=-a_{N} w_{1}^{N}$. We need to find $\xi_{2}=\left(z_{2}, w_{2}\right) \in \mathfrak{C}_{0, N}$ which is compatible with $\xi_{1}$ in the sens of Definition 10.4. By (8.6) and (8.8), the membership $\xi_{2}=\left(z_{2}, w_{2}\right) \in \mathfrak{C}_{0, N}$ is equivalent to $z_{N}(z, w)=0$. Therefore, applying Proposition 10.3 (i) to $r=0$, we may find a unique $z=f(w)$ such that $|z| \leq|w|$ and that $z_{N}(z, w)=0$. Clearly, $\mathbb{D}_{s} \ni w \mapsto f(w)$ is a holomorphic function. Using the function $\theta$ given in (8.2), we introduce the following holomorphic function

$$
\theta_{N}(w):=\theta(Z(f(w), w), W(f(w), w)), \quad \text { for } \quad w \in \mathbb{D}_{s_{0}} .
$$

By (8.3), we get that

$$
1 / 2<\left|\theta_{N}(w)\right|<2 \text { and } \lim _{w \rightarrow 0} \theta_{N}(w)=\theta_{N}(0)=1,
$$

the limit being uniform in $N$. By Proposition 10.3 (ii) with $r=0$ and (8.2), we may write

$$
f(w)=\theta_{N}(w)\left(-a_{N} w^{N}+g(w)\right) \quad \text { for } \quad w \in \mathbb{D}_{s_{0}},
$$

where $g$ is a holomorphic function on $\mathbb{D}_{s_{0}}$ which satisfies $|g(w)| \leq M^{-1}\left|a_{N} w^{N}\right|$, $w \in \mathbb{D}_{s_{0}}$.

In order to find $\xi_{2}=\left(z_{2}, w_{2}\right) \in \mathfrak{C}_{0, N}$ which is compatible with $\xi_{1}$, we write $z_{2}=e^{t} z_{1}, w_{2}=e^{\lambda t} w_{1}$ for some $0<|t| \ll 1$. We deduce from this and from $\left(z_{2}, w_{2}\right) \in \mathfrak{C}_{0, N}$ that $f\left(e^{\lambda t} w_{1}\right)=e^{t} z_{1}$. Since $z_{1}=-a_{N} w_{1}^{N}$, it follows that $t$ is a root of the following holomorphic function on the disc $\mathbb{D}_{s}, s \in\left(0, s_{0}\right)$ being a number whose exact value will be determined later on:

$$
F(t):=-a_{N} e^{\lambda N t} w_{1}^{N}+g\left(e^{\lambda t} w_{1}\right)+a_{N} e^{t} w_{1}^{N} \theta_{N}^{-1}\left(e^{\lambda t} w_{1}\right), \quad t \in \mathbb{D}_{s} .
$$


Consider the holomorphic function

$$
H(t):=-a_{N} e^{\lambda N t} w_{1}^{N}+a_{N} e^{t} w_{1}^{N}, \quad t \in \mathbb{D}_{s} .
$$

Observe that $H(t)=0$ if and only if $t=\frac{2 i \pi k}{\lambda N-1}$ for $k \in \mathbb{Z}$. So we choose the constant $s$ as follows:

$$
s=c^{\prime} \frac{\pi}{|\lambda N-1|} \quad \text { for } c^{\prime}>0 \text { a constant independent of } N, r .
$$

Hence $H$ has the unique root $t=0$ on $\mathbb{D}_{s}$. On the other hand, observe that

$$
H(t)=a_{N} w_{1}^{N}\left((-\lambda N+1) t+O\left(t^{2}\right)\right), \quad \text { where } O\left(t^{2}\right) \text { depends on } N \text {. }
$$

Consequently, when the constant $c^{\prime}$ (being independent of $N, r$ ) is small enough,

$$
|H(t)| \approx\left|a_{N} w_{1}^{N}\right| \text { and }\left|a_{N} w_{1}^{N}\right|<|H(t)| \text { for } t \in \partial \mathbb{D}_{s} .
$$

Using this, we can show that for $N$ large enough and $t \in \partial \mathbb{D}_{s}$,

$$
\begin{aligned}
|F(t)-H(t)| & \leq\left|g\left(e^{\lambda t} w_{1}\right)\right|+\left|a_{N} e^{t} w_{1}^{N}\right|\left|\theta_{N}^{-1}\left(e^{\lambda t} w_{1}\right)-1\right| \\
& \ll\left|a_{N} w_{1}^{N}\right|<|H(t)|,
\end{aligned}
$$

where the first inequality holds by the uniform limit (with respect to $N$ ) in (10.18) and the estimate $\left|g\left(e^{\lambda t} w_{1}\right)\right| \leq M^{-1}\left|a_{N} e^{\lambda N t} w_{1}^{N}\right|$.

So $|G(t)-H(t)|<H(t)$ on $\partial \mathbb{D}_{s}$, and hence by Rouché's theorem, $G$ has a unique root on $\mathbb{D}_{s}$. Consequently, there is a unique $t \in \mathbb{D}_{s}$ such that $F(t)=0$, i.e., there is a unique $\xi_{2}=\left(e^{t} z_{1}, e^{\lambda t} w_{1}\right) \in \mathfrak{C}_{0, N}$ with $|t| \leq s$. Since $s \approx N^{-1}$, (10.17) follows from Lemma 10.5.

Lemma 10.7. Let $N \in \mathbb{N}$ be as above and $\alpha \in \mathbb{T}$. Let $\xi_{0, N, \alpha, k}(k \in \mathbb{N})$ be the intersection points of $\mathfrak{C}_{0, N}$ with the Riemann surface $\widehat{L}_{\alpha}$ described by Lemma 10.6 Then there is a constant $c_{N}>1$ independent of $\alpha$ satisfying the following properties for every $0<r<r_{N}$ :

(i) the intersection of the curve $\mathfrak{C}_{r, N}$ with the Riemann surface $\widehat{L}_{\alpha}$ inside $\left(r_{N} \mathbb{D}\right)^{2} \backslash \mathbb{B}_{r^{1 / N}|\log r|^{3 / N}}$ can be enumerated as $\xi_{r, N, \alpha, k}$ such that $\xi_{r, N, \alpha, k}$ and $\xi_{0, N, \alpha, k}$ are compatible, where $k \in \mathbb{N}$ such that $\xi_{0, N, \alpha, k} \in\left(r_{N} \mathbb{D}\right)^{2} \backslash \mathbb{B}_{r^{1 / N}|\log r|^{3 / N}}$;

(ii) for every $k \in \mathbb{N}$ with $\xi_{0, N, \alpha, k} \in\left(r_{N} \mathbb{D}\right)^{2} \backslash \mathbb{B}_{r^{1 / N}|\log r|^{3 / N}}$,

$$
\operatorname{dist}_{C}\left(\xi_{r, N, \alpha, k}, \xi_{0, N, \alpha, k}\right) \leq c_{N}|\log r|^{-3} .
$$

Proof. We need to prove that for every point

$$
\begin{gathered}
\xi_{1} \in\left(\mathfrak{C}_{0, N} \cap \widehat{L}_{\alpha}\right) \cap\left(\left(r_{N} \mathbb{D}\right)^{2} \backslash \mathbb{B}_{r^{1 / N}|\log r|^{3 / N}}\right) \\
\text { (resp. } \quad \xi_{1} \in\left(\mathfrak{C}_{r, N} \cap \widehat{L}_{\alpha}\right) \cap\left(\left(r_{N} \mathbb{D}\right)^{2} \backslash \mathbb{B}_{r^{1 / N}|\log r|^{3 / N}}\right) \quad \text { ), }
\end{gathered}
$$

there is exactly one point

$$
\left.\begin{array}{c}
\xi_{2} \in\left(\mathfrak{C}_{r, N} \cap \widehat{L}_{\alpha}\right) \cap\left(\left(r_{N} \mathbb{D}\right)^{2} \backslash \mathbb{B}_{r^{1 / N}|\log r|^{3 / N}}\right) \\
\left(\text { resp. } \quad \xi_{2} \in\left(\mathfrak{C}_{0, N} \cap \widehat{L}_{\alpha}\right) \cap\left(\left(r_{N} \mathbb{D}\right)^{2} \backslash \mathbb{B}_{r^{1 / N}|\log r|^{3 / N}}\right)\right.
\end{array}\right)
$$


such that $\xi_{1}$ and $\xi_{2}$ are compatible and that

$$
\operatorname{dist}_{C}\left(\xi_{1}, \xi_{2}\right) \lesssim|\log r|^{-3} \text {. }
$$

We will only show that for every point

$$
\xi_{1} \in\left(\mathfrak{C}_{0, N} \cap \widehat{L}_{\alpha}\right) \cap\left(\left(r_{N} \mathbb{D}\right)^{2} \backslash \mathbb{B}_{r^{1 / N}|\log r|^{3 / N}}\right),
$$

there is exactly one point

$$
\xi_{2} \in\left(\mathfrak{C}_{r, N} \cap \widehat{L}_{\alpha}\right) \cap\left(\left(r_{N} \mathbb{D}\right)^{2} \backslash \mathbb{B}_{r^{1 / N}|\log r|^{3 / N}}\right)
$$

satisfying (10.21) since the other assertion can be proved similarly. Let $s_{0}:=r_{N}$.

Let $f$ and $g$ be the holomorphic functions on $\mathbb{D}_{s_{0}}$ introduced in the proof of Lemma 10.6 (see (10.19)). Since $g$ satisfies $|g(w)| \leq M^{-1}\left|a_{N} w^{N}\right|, w \in \mathbb{D}_{s_{0}}, f$ admits the following Taylor expansion:

$$
f(w)=-\tilde{a}_{N} w^{N}+h(w) \quad \text { for } \quad w \in \mathbb{D}_{s_{0}},
$$

where $h(w)=O\left(w^{N+1}\right)$. By (10.16) we get that

$$
\left|\tilde{a}_{N} / a_{N}-1\right|<2^{-N} \quad \text { for } N \text { large enough. }
$$

Write $\xi_{1}=\left(z_{1}, w_{1}\right)$. Since $\xi_{1} \in \mathfrak{C}_{0, N}$, the previous lemma implies that $z_{1}=f\left(w_{1}\right)$. Recall from Section 8 the coordinates $(Z, W)$. Under the coordinates $(Z, W)$, we infer from (8.6), (8.7) and (8.8) the following simple correspondence between $\mathfrak{C}_{0, N}$ and $\mathfrak{C}_{r, N}$ :

$$
(Z, W) \in \mathfrak{C}_{0, N} \quad \Longleftrightarrow \quad(r+Z, W) \in \mathfrak{C}_{r, N} .
$$

In order to exploit this nice correspondence under the coordinates $(z, w)$, we introduce the holomorphic function $R_{r}$ given by the following relation

$$
Z\left(R_{r}(w)+f(w), w\right)-Z(f(w), w)=r, \quad w \in \mathbb{D}_{s_{0}} .
$$

Recall from (8.1) that the Jacobian matrix of $(Z, W)$ over $(z, w)$ at $(0,0)$ is the identity matrix. Consequently, using the Taylor expansion of $Z(z, w)$ and substituting $f(w)$ (resp. $R_{r}(w)+f(w)$ ) for $z$, we infer from (10.25) that

$$
R_{r}(w)+O\left(R_{r}^{2}(w)\right)+O\left(R_{r}(w) f(w)\right)=r, \quad \text { for } \quad w \in \mathbb{D}_{s_{0}} .
$$

We need to find $\xi_{2}=\left(z_{2}, w_{2}\right) \in \mathfrak{C}_{r, N}$ which is compatible with $\xi_{1}$. Write $z_{2}=e^{t} z_{1}, w_{2}=e^{\lambda t} w_{1}$ for some $0<|t| \ll 1$. We deduce from this and from $\left(z_{2}, w_{2}\right) \in \mathfrak{C}_{r, N}$ and (10.25) that

$$
R_{r}\left(e^{\lambda t} w_{1}\right)+f\left(e^{\lambda t} w_{1}\right)=e^{t} z_{1} .
$$

In the sequel, $s \in\left(0, s_{0}\right)$ is a number whose exact value will be determined later on. Since $z_{1}=f\left(w_{1}\right)$, it follows from the last line and (10.22) that $t$ is a root of the following holomorphic function on $\mathbb{D}_{s}$ defined by

$$
F(t):=R_{r}\left(e^{\lambda t} w_{1}\right)-\tilde{a}_{N} e^{\lambda N t} w_{1}^{N}+h\left(e^{\lambda t} w_{1}\right)+\tilde{a}_{N} e^{t} w_{1}^{N}-e^{t} h\left(w_{1}\right), \quad t \in \mathbb{D}_{s} .
$$


On the other hand, since $\left(z_{1}, w_{1}\right) \in \mathfrak{C}_{0, N}$, we get by Proposition 10.3 with $r=0$ that $2\left|a_{N} w_{1}^{N}\right| \geq\left|z_{1}\right|$. This together with the second inequality in (10.9) imply $\left|z_{1}\right| \leq 2^{N+1}\left|w_{1}\right|$. Since $\left(z_{1}, w_{1}\right) \notin \mathbb{B}_{r^{1 / N}|\log r|^{3 / N}}$, it follows that $\left|w_{1}\right| \geq$ $2^{-N-1} r^{1 / N}|\log r|^{3 / N}$. This together with the first inequality in (10.9) yield that

$$
\left|a_{N} w_{1}^{N}\right| \geq 2^{-N}\left|w_{1}\right|^{N} \geq 2^{-N(N+1)} r|\log r|^{3} .
$$

Hence, there is $c_{N}>1$ such that

$$
r<c_{N}|\log r|^{-3}\left|a_{N} w_{1}^{N}\right| .
$$

Now we choose $M$ large enough ( $M$ depending on $N$ ), and $0<s<s_{0}$ such that

(10.30) $s:=c^{\prime}|\log r|^{-3} \quad$ for $c^{\prime}=c_{N}^{\prime}>0$ a large constant independent of $r$.

Then we deduce from (10.29), (10.30) and (10.26), (10.18), (10.19) and (10.23) that for $r>0$ small enough,

$$
R_{r}(w)=r+o(r) \quad \text { and } \quad r \ll s\left|\tilde{a}_{N} w_{1}^{N}\right| .
$$

Consider the holomorphic function

$$
H(t):=-\tilde{a}_{N} e^{\lambda N t} w_{1}^{N}+\tilde{a}_{N} e^{t} w_{1}^{N}, \quad t \in \mathbb{D}_{s} .
$$

Observe that $H$ has the unique root $t=0$ on $\mathbb{D}_{s}$. Moreover, when the constant $c^{\prime}$ is large enough, we have that

$$
|H(t)| \approx s\left|\tilde{a}_{N} w_{1}^{N}\right| \quad \text { and } \quad|H(t)|>s\left|\tilde{a}_{N} w_{1}^{N}\right|, \quad \text { for } \quad t \in \partial \mathbb{D}_{s} .
$$

We also infer from (10.22) that

$$
h\left(e^{\lambda t} w\right)-e^{t} h(w)=O\left(t w^{N+1}\right) \quad \text { for } \quad w \in \mathbb{D}_{s_{0}},
$$

where $O(\cdot)$ depends on $N$.

Putting this together with the definition of $F$ and $H$ and (10.32) and (10.31), a straightforward computation shows that for $t \in \partial \mathbb{D}_{s}$,

$$
\begin{aligned}
|F(t)-H(t)| & \left.\leq\left|R_{r}\left(e^{\lambda t} w_{1}\right)\right|+\mid h\left(e^{\lambda t} w_{1}\right)\right)-e^{t} h\left(w_{1}\right) \mid \\
& \leq s\left|\tilde{a}_{N} w_{1}^{N}\right|<|H(t)| .
\end{aligned}
$$

Using this, we can apply Rouché's theorem to $F$ and $H$. Consequently, $F$ has a unique root on $\mathbb{D}_{s}$. Therefore, there is a unique $t \in \mathbb{D}_{s}$ such that $F(t)=0$, i.e., there is a unique $\xi_{2}=\left(e^{t} z_{1}, e^{\lambda t} w_{1}\right) \in \mathfrak{C}_{r, N}$ with $|t| \leq s$. Since $s \approx|\log r|^{-3}$, (10.21) follows from Lemma 10.5.

In order to prove the last part of Proposition 9.1, the following lemma gives us the discrepancy between the intersection points of a leaf with the algebraic curve $\mathfrak{C}_{r, N}^{*}$ and with the analytic curve $\mathfrak{C}_{r}$ inside the ball $\mathbb{B}_{r^{1 / N}|\log r|^{-3 / N}}$. 
Lemma 10.8. Let $N \in \mathbb{N}$ be as above and $\alpha \in \mathbb{T}$. Let $\xi_{r, \alpha, k}(k \in \mathbb{N})$ be the intersection of the analytic curve $\mathfrak{C}_{r}$ with the Riemann surface $\widehat{L}_{\alpha}$ described by Lemma 10.1. Then there is a constant $c_{N}>1$ large enough independent of $\alpha$ satisfying the following properties for every $0<r<r_{N}$ :

(i) the intersection of the curve $\mathfrak{C}_{r, N}$ with the Riemann surface $\widehat{L}_{\alpha}$ inside the ball $\mathbb{B}_{r^{1 / N}|\log r|^{-3 / N}}$ can be enumerated as $\xi_{r, N, \alpha, k}$ such that $\xi_{r, N, \alpha, k}$ and $\xi_{r, \alpha, k}$ are compatible, where $k \in \mathbb{N}$ such that $\xi_{r, \alpha, k} \in \mathbb{B}_{r^{1 / N}|\log r|^{-3 / N}}$;

(ii) for every $k \in \mathbb{N}$ with $\xi_{r, \alpha, k} \in \mathbb{B}_{r^{1 / N}|\log r|^{-3 / N}}$, we have that

$$
\operatorname{dist}_{C}\left(\xi_{r, N, \alpha, k}, \xi_{r, \alpha, k}\right) \leq c_{N}|\log r|^{-3} .
$$

Proof. We need to prove that for every point $\xi_{1} \in\left(\mathfrak{C}_{r} \cap \widehat{L}_{\alpha}\right) \cap \mathbb{B}_{r^{1 / N}|\log r|^{-3 / N}}$ (resp. $\left.\xi_{1} \in\left(\mathfrak{C}_{r, N} \cap \widehat{L}_{\alpha}\right) \cap \mathbb{B}_{r^{1 / N}|\log r|^{-3 / N}}\right)$, there is exactly one point $\xi_{2} \in\left(\mathfrak{C}_{r, N} \cap \widehat{L}_{\alpha}\right) \cap$ $\mathbb{B}_{r^{1 / N}|\log r|^{-3 / N}}$ (resp. $\left.\xi_{2} \in\left(\mathfrak{C}_{r} \cap \widehat{L}_{\alpha}\right) \cap \mathbb{B}_{r^{1 / N}|\log r|^{-3 / N}}\right)$ such that $\xi_{1}$ and $\xi_{2}$ are compatible and that

$$
\operatorname{dist}_{C}\left(\xi_{1}, \xi_{2}\right) \lesssim|\log r|^{-3} \text {. }
$$

We will only show that for every point $\xi_{1} \in\left(\mathfrak{C}_{r} \cap \widehat{L}_{\alpha}\right) \cap \mathbb{B}_{r^{1 / N}|\log r|^{-3 / N}}$, there is exactly one point $\xi_{2} \in\left(\mathfrak{C}_{r, N} \cap \widehat{L}_{\alpha}\right) \cap \mathbb{B}_{r^{1 / N}|\log r|^{-3 / N}}$ satisfying (10.34) since the other assertion can be proved similarly.

Write $\xi_{1}=\left(z_{1}, w_{1}\right)$. So $z_{1}=r$. We need to find $\xi_{2}=\left(z_{2}, w_{2}\right) \in \mathfrak{C}_{r, N}$ which is compatible with $\xi_{1}$. Let $s_{0}:=r_{N}$. Consider two cases.

Case 1: $\left|w_{1}\right| \geq 2 r$.

In this case we fix a number $s \in\left(0, s_{0}\right)$ as follows

$$
s:=c^{\prime}|\log r|^{-3} \quad \text { for } c^{\prime}>0 \text { a large constant independent of } r .
$$

Let $f, h$ and $R_{r}$ be the holomorphic functions on $\mathbb{D}_{s_{0}}$ introduced in the proof of Lemma 10.6 and Lemma 10.7 (see (10.22) and (10.25)). On the other hand, we deduce from the membership $\left(z_{2}, w_{2}\right) \in \mathfrak{C}_{r, N}$ and (10.24) and (10.25) that $z_{2}=R_{r}\left(w_{2}\right)+f\left(w_{2}\right)$. Write $z_{2}=e^{t} z_{1}, w_{2}=e^{\lambda t} w_{1}$ for some $0<|t| \ll 1$ since $\xi_{2}$ is compatible with $\xi_{1}$. Consequently, we infer that $t$ is a solution of the following equation

$$
R_{r}\left(e^{\lambda t} w_{1}\right)+f\left(e^{\lambda t} w_{1}\right)=e^{t} z_{1} .
$$

Using (10.22) and the equality $z_{1}=r$, we deduce that $t$ is a root of the following holomorphic function on the disc $\mathbb{D}_{s}$

$$
F(t):=R_{r}\left(e^{\lambda t} w_{1}\right)-\tilde{a}_{N} e^{\lambda N t} w_{1}^{N}+h\left(e^{\lambda t} w_{1}\right)-e^{t} r, \quad t \in \mathbb{D}_{s} .
$$

Consider another holomorphic function on $\mathbb{D}_{s}$ :

$$
H(t):=r-r e^{t}, \quad t \in \mathbb{D}_{s} .
$$

Observe that $H$ admits a unique root $t=0$ on $\mathbb{D}_{s}$. Moreover, $|H(t)| \approx s r$ for $t \in \partial \mathbb{D}_{s}$. 
Since $\left(z_{1}, w_{1}\right) \in \mathbb{B}_{r^{1 / N}|\log r|^{-3 / N}}$, it follows that $\left|w_{1}\right| \leq r^{1 / N}|\log r|^{-3 / N}$. This together with the second inequality in (10.9) yield that

$$
\left|a_{N} w_{1}^{N}\right| \leq 2^{N}\left|w_{1}\right|^{N} \leq 2^{N} r|\log r|^{-3} .
$$

This, combined with (10.19) and (10.22), implies that when the constant $c^{\prime}$ is large enough,

$$
\left|\tilde{a}_{N} w_{1}^{N}\right|<s r / 2 .
$$

Then we deduce from (10.35) and (10.26), (10.18), (10.19) that for $r>0$ small enough,

$$
R_{r}(w)=r+O\left(r^{2}\right), \quad w \in \mathbb{D}_{s_{0}} .
$$

On the other hand, we infer from (10.22) that $\left|h\left(e^{\lambda t} w_{1}\right)\right| \ll\left|\tilde{a}_{N} e^{\lambda N t} w_{1}^{N}\right|$. Putting this together with (10.35) and (10.36), we deduce for $t \in \partial \mathbb{D}_{s}$ that

$$
\begin{aligned}
|F(t)-H(t)| & \leq\left|R_{r}\left(e^{\lambda t} w_{1}\right)-r\right|+\left|h\left(e^{\lambda t} w_{1}\right)\right| \\
& \leq O\left(r^{2}\right)+\left|\tilde{a}_{N} w_{1}^{N}\right|<s r \approx|H(t)| .
\end{aligned}
$$

Consequently, by Rouché's theorem applied to $F$ and $H$, there is a unique $t \in \mathbb{D}_{s}$ such that $F(t)=0$, i.e., there is a unique $\xi_{2}=\left(e^{t} z_{1}, e^{\lambda t} w_{1}\right) \in \mathfrak{C}_{r, N}$ with $|t| \leq s$. Since $s \lesssim|\log r|^{-3}$, (10.34) follows from Lemma10.5.

Case 2: $\left|w_{1}\right| \leq 2 r$.

Here the difficulty lies in the fact that we cannot apply Proposition 10.3 (i) and that the functions $f, g, h$ etc are therefore not available any more. In this case we choose $s:=c^{\prime} r^{2}$ for $c^{\prime}>0$ a large constant. Using the assumption $\left|w_{1}\right| \leq 2 r$ and the expansion (10.10), we get that

$$
\left|z_{\infty}\left(r, w_{1}\right)-z_{N}\left(r, w_{1}\right)\right| \leq \sum_{j=N}^{\infty}\left|a_{j}\right|\left|\vartheta^{j}\left(r, w_{1}\right)\right|\left(\left|w_{1}\right|+\left|\sum_{k=2}^{\infty} b_{k} r^{k}\right|\right)^{j}=O\left(r^{2}\right) .
$$

Moreover, using (8.2) and (8.1) we infer that

$$
\left|r-z_{\infty}\left(r, w_{1}\right)\right|=O\left(r^{2}\right) .
$$

On the other hand, using (10.10) and (8.2) and (8.1), we obtain that

$$
\begin{aligned}
z_{N}\left(e^{t} r, e^{\lambda t} w_{1}\right)-z_{N}\left(r, w_{1}\right) & =z_{\infty}\left(e^{t} r, e^{\lambda t} w_{1}\right)-z_{\infty}\left(r, w_{1}\right)+O\left(r^{2}\right)+O\left(t^{2}\right) \\
& =e^{t} r-r+O\left(r^{2}\right)+O\left(t^{2}\right)=O\left(r^{2}\right)+O\left(t^{2}\right) .
\end{aligned}
$$

Putting together these three estimates, we have for $t \in \mathbb{D}_{s}$ that

$$
\begin{aligned}
\left|z_{N}\left(e^{t} r, e^{\lambda t} w_{1}\right)-r\right| & \leq\left|z_{N}\left(e^{t} r, e^{\lambda t} w_{1}\right)-z_{N}\left(r, w_{1}\right)\right|+\left|z_{N}\left(r, w_{1}\right)-z_{\infty}\left(r, w_{1}\right)\right| \\
& +\left|r-z_{\infty}\left(r, w_{1}\right)\right| \\
& =t+O\left(t^{2}\right)+O\left(r^{2}\right) .
\end{aligned}
$$

Consequently, when $c^{\prime}$ is large enough, we know by Rouché's theorem applied to the identity function $\mathbb{D}_{s} \ni t \mapsto t$ and the holomorphic function $t \mapsto$ $z_{N}\left(e^{t} r, e^{\lambda t} w_{1}\right)-r$ that the latter admits a unique root on $\mathbb{D}_{s}$. Hence, there is a 
unique $\xi_{2}=\left(e^{t} z_{1}, e^{\lambda t} w_{1}\right) \in \mathfrak{C}_{r, N}$ with $|t| \leq s$. Since $s \lesssim|\log r|^{-3}$, (10.34) follows from Lemma 10.5 ,

\section{MASS OF $T \wedge\left[\mathfrak{C}_{r}\right]$ ON BALLS}

The main purpose of this section is to prove Proposition 8.3 and one half of Proposition 8.6. Recall that $\mathbb{T}:=\left\{\alpha \in \mathbb{C}: e^{-2 \pi b} \leq|\alpha| \leq 1\right\}$. In parallel with the integral operator $K_{s}$ given in (6.3), we also consider, for each $s>0$, the domain $D_{s}:=\{t \in \mathbb{R}: t \geq s\}$, and the function $\widetilde{K}_{s}: \mathbb{R} \rightarrow \mathbb{R}^{+}$given by

$$
\widetilde{K}_{s}(y):=\int_{D_{s}} \frac{V}{V^{2}+(y-U)^{2}} d t, \quad y \in \mathbb{R} .
$$

Here $U, V$ are functions of the variable $t$ and the parameter $s$ which satisfy the following system of equations (see (5.6), (5.2) and (5.3)):

$$
U+i V=(u+i s)^{\gamma} \quad \text { and } \quad t=b u+a s .
$$
8.3 .

The following result is the main technical point in the proof of Proposition

Lemma 11.1. There is a constant $c>1$ such that for all $y \in \mathbb{R}$ and $s>0$,

$$
c^{-1} \leq \widetilde{K}_{s}(y) / K_{s}(y) \leq c .
$$

Taking Lemma 11.1 for granted, we arrive at the

End of the proof of Proposition 8.3. In what follows we use the notation introduced in Lemma 10.1. By (10.1), we have that $u_{\alpha, k}=2 k \pi-(\log |\alpha|) / b$ and $v_{r}=-\log r$. This, coupled with (5.1), (5.2) and (5.3), implies that $\xi_{r, \alpha, k} \in \mathbb{B}_{r^{s}}$ if and only if $b u_{\alpha, k}+a v_{r} \geq-\delta \log r$, which is, in turn, equivalent to

$$
k \geq \frac{1}{2 \pi b}((\delta-a)(-\log r)+\log |\alpha|) .
$$

Let $\mathbb{Z}_{\delta, r, \alpha}$ be the set of all integers $k$ satisfying the last inequality. Observe that

$$
\left\|T \wedge\left[\mathfrak{C}_{r}\right]\right\|_{\mathbb{B}_{r} \delta}=\int_{\mathbb{T}}\left(\sum_{k \in \mathbb{Z}_{\delta, r, \alpha}} h_{\alpha}\left(\xi_{r, \alpha, k}\right)\right) d \nu(\alpha) .
$$

For $\alpha \in \mathbb{T}$ let $D^{\alpha}:=D_{-\delta \log r}, t_{r, \alpha, k}:=b u_{\alpha, k}+a v_{r}, k \in \mathbb{Z}$. Then $k \in \mathbb{Z}_{\delta, r, \alpha}$ if and only if $t_{r, \alpha, k} \in D^{\alpha}$. Moreover, consider the function $\chi^{\alpha}: D^{\alpha} \rightarrow \mathbb{C}$ given by

$$
\chi^{\alpha}(t):=u+i v_{r}, \quad \text { where } \quad v_{r}=-\log r \quad \text { and } \quad t=b u+a v_{r} .
$$

Let $m=1$ and choose $\rho>1$ large enough. Using Harnack's inequality, we see that the assumption of Part 1) of Proposition 7.7 is fulfilled. Hence, by Definition 7.8 we obtain an interpretation $\left(K^{\alpha}\right)_{\alpha \in \mathbb{T}}$ of the geometric intersection $T \wedge\left[\mathfrak{C}_{r}\right]$ on $\mathbb{B}_{r^{\delta}}$ with mesh $m=1$. Moreover, we infer from the above discussion and formula (11.1) that $K^{\alpha}(y)=\widetilde{K}_{-\delta \log r}(y)$. Consequently, by Part 1) of 
Proposition 7.7 we get that

$$
\left\|T \wedge\left[\mathfrak{C}_{r}\right]\right\|_{\mathbb{B}_{r} \delta} \approx \int_{\alpha \in \mathbb{T}}\left(\int_{-\infty}^{\infty} \widetilde{K}_{-\delta \log r}(y) \tilde{H}_{\alpha}(y) d y\right) d \nu(\alpha) .
$$

On the other hand, by Lemma 11.1 we know that $\widetilde{K}_{-\delta \log r}(y) \approx K_{-\delta \log r}(y)$. By the definition of $K_{s}$ in (6.3), we may find a constant $c_{\delta}>1$ such that $c_{\delta}^{-1}<$ $K_{-\delta \log r}(y) / K_{-\delta \log r}(y)<c_{\delta}$. So $\widetilde{K}_{-\delta \log r}(y) \approx K_{-\log r}(y)$. This, combined with the last estimate for $\left\|T \wedge\left[\mathfrak{C}_{r}\right]\right\|_{\mathbb{B}_{r} \delta}$, implies that

$$
\left\|T \wedge\left[\mathfrak{C}_{r}\right]\right\|_{\mathbb{B}_{r^{\delta}}} \approx \int_{\alpha \in \mathbb{T}}\left(\int_{-\infty}^{\infty} K_{-\log r}(y) \tilde{H}_{\alpha}(y) d y\right) d \nu(\alpha) .
$$

Comparing this with Lemma 6.1, the proof is completed.

End of the proof of Lemma 11.1, Let $c_{2}, c_{3}$ be the constants with $c_{3}>c_{2}>1$ given by Lemma 7.9. We consider three cases.

Case 1: $s \geq c_{2}(1+|y|)^{1 / \gamma}$.

By Part 2) of Lemma 7.9 and by formula (11.1), we have that

$$
\widetilde{K}_{s}(y) \approx \int_{t=s}^{\infty} \frac{s d t}{t^{\gamma+1}} \approx s^{1-\gamma} .
$$

This, compared with formula (6.3), completes the proof of Case 1 .

Case 2: $c_{3}^{-1} \leq \frac{s}{(1+|y|)^{1 / \gamma}} \leq c_{2}$.

Write $D_{s}=D_{s}^{1} \cup D_{s}^{2}$, where

$$
\begin{aligned}
& D_{s}^{1}:=\left\{t \in D_{s}: t \leq c_{2}(1+|y|)^{1 / \gamma}\right\}, \\
& D_{s}^{2}:=\left\{t \in D_{s}: t \geq c_{2}(1+|y|)^{1 / \gamma}\right\} .
\end{aligned}
$$

Consequently, formula (11.1) gives that

$$
\widetilde{K}_{s}(y)=\left(\int_{D_{s}^{1}}+\int_{D_{s}^{2}}\right) \frac{V}{V^{2}+(y-U)^{2}} d t=: I+I I .
$$

To estimate $(I)$, we apply Part 4) of Lemma 7.9 and obtain that

$$
I \approx \int_{D_{s}^{1}} \frac{d t}{(1+|y|)}=\int_{c_{3}^{-1}(1+|y|)^{1 / \gamma}}^{c_{2}(1+|y|)^{1 / \gamma}} \frac{d t}{(1+|y|)} \approx(1+|y|)^{1 / \gamma-1} .
$$

To estimate (II), we apply Part 2) of Lemma 7.9 and obtain that

$$
I I \approx \int_{D_{s}^{2}} \frac{\min \{t, v\} d t}{(\max \{t, v\})^{\gamma+1}} \leq \int_{c_{3}^{-1}(1+|y|)^{1 / \gamma}}^{\infty} \frac{s d t}{t^{\gamma+1}} \approx(1+|y|)^{1 / \gamma-1} .
$$

Inserting the above estimates for $(I)$ and $(I I)$ into (11.2), we obtain that $\widetilde{K}_{s}(y) \approx$ $(1+|y|)^{1 / \gamma-1}$ in the second case. Comparing this with formula (6.3), the proof of Case 2 is complete.

Case 3: $s \leq c_{3}^{-1}(1+|y|)^{1 / \gamma}$. 
Write $D_{s}=D_{s}^{1} \cup D_{s}^{2} \cup D_{s}^{3}$, where

$$
\begin{aligned}
& D_{s}^{1}:=\left\{t: s \leq t \leq c_{2}^{-1}(1+|y|)^{1 / \gamma}\right\}, \\
& D_{s}^{2}:=\left\{t: t \geq c_{2}(1+|y|)^{1 / \gamma}\right\}, \\
& D_{s}^{3}:=\left\{t: c_{2}^{-1}(1+|y|)^{1 / \gamma} \leq t \leq c_{2}(1+|y|)^{1 / \gamma}\right\} .
\end{aligned}
$$

Consequently, we get, similarly as in (11.2), that

$$
\widetilde{K}_{s}(y)=\left(\int_{D_{s}^{1}}+\int_{D_{s}^{2}}+\int_{D_{s}^{3}}\right) \frac{V}{V^{2}+(y-U)^{2}} d t=: I+I I+I I I .
$$

To estimate $(I)$ we apply Part 1) and Part 3) of Lemma 7.9. Consequently, we obtain that

$$
I \approx \int_{D_{s}^{1}} \frac{t^{\gamma-1} s d t}{(1+|y|)^{2}} \approx s\left(\int_{t=s}^{c_{2}^{-1}(1+|y|)^{1 / \gamma}} \frac{t^{\gamma-1} d t}{(1+|y|)^{2}}\right) \lesssim s(1+|y|)^{-1} .
$$

To estimate $(I I)$, we apply Part 2) of Lemma 7.9 and obtain that

$$
I I \approx \int_{D_{s}^{2}} \frac{s d t}{t^{\gamma+1}} \approx s\left(\int_{c_{2}(1+|y|)^{1 / \gamma}}^{\infty} \frac{d t}{t^{\gamma+1}}\right) \approx s(1+|y|)^{-1}
$$

To estimate $(I I I)$, we apply Part 5) of Lemma 7.9 and obtain that

$$
I I I \approx \int_{c_{2}^{-1}(1+|y|)^{1 / \gamma}}^{c_{2}(1+|y|)^{1 / \gamma}} \frac{(1+|y|)^{1 / \gamma-1} s d t}{s^{2}+(t-\rho(y, s))^{2}}
$$

where $\rho(y, s)$ satisfies $c_{2}^{-1}(1+|y|)^{1 / \gamma} \leq \rho(y, s) \leq c_{2}(1+|y|)^{1 / \gamma}$. Write $I I I:=$ $I I I_{1}+I I I_{2}$, where

$I I I_{1}=\int_{|t-\rho(y, s)| \leq s} \frac{(1+|y|)^{1 / \gamma-1} s d t}{s^{2}+(t-\rho(y, s))^{2}} \approx \int_{|t-\rho(y, s)| \leq s} \frac{(1+|y|)^{1 / \gamma-1} d t}{s} \approx(1+|y|)^{1 / \gamma-1}$,

and

$$
I I I_{2} \approx \int \frac{(1+|y|)^{1 / \gamma-1} s d t}{s^{2}+(t-\rho(y, s))^{2}} \leq \int \frac{(1+|y|)^{1 / \gamma-1} s d t}{(t-\rho(y, s))^{2}} \lesssim c(1+|y|)^{1 / \gamma-1}
$$

the integrals in the last line being taken over the region

$$
\left\{t \in \mathbb{R}: c_{2}^{-1}(1+|y|)^{1 / \gamma} \leq t \leq c_{2}(1+|y|)^{1 / \gamma} \text { and }|t-\rho(y, s)| \geq s\right\} .
$$

Thus, $I I I \approx(1+|y|)^{1 / \gamma-1}$.

Combining the obtained estimates for $(I),(I I)$ and $(I I I)$, and using the assumption $s \leq c_{3}^{-1}(1+|y|)^{1 / \gamma}$, we infer that

$$
\widetilde{K}_{s}(y)=I+I I+I I I \approx s(1+|y|)^{-1}+(1+|y|)^{1 / \gamma-1} \approx(1+|y|)^{1 / \gamma-1} .
$$

This, compared with formula (6.3), allows us to conclude the proof of the last case.

As an application of Lemma 11.1, we are able to establish one half of the proof of Proposition 8.6. 
Proof of Proposition 8.6 for the geometric intersection $T \wedge\left[\mathfrak{C}_{r}\right]$. In parallel with the integral operator $\widetilde{K}_{s}$ given in (11.1), we consider, for each $s>0$, the domain $D_{s, N}:=\{t \in \mathbb{R}: t \geq s / N+3(\log s) / N\}$, and the function $K_{s, N}: \mathbb{R} \rightarrow$ $\mathbb{R}^{+}$given by

$$
K_{s, N}(y):=\int_{D_{s, N}} \frac{V}{V^{2}+(y-U)^{2}} d t, \quad y \in \mathbb{R},
$$

Here $U, V$ are functions of the variable $t$ and the parameter $s$ which satisfy the following system of equations (see (5.6), (5.2) and (5.3)):

$$
U+i V=(u+i s)^{\gamma} \quad \text { and } \quad t=b u+a s .
$$

We argue as in the proof of Lemma 11.1 for $\delta:=1 / N$ making the obviously necessary changes. The factor $\log s$ in the definition of $D_{s, N}$ can be overlooked without changing the final result. For $\alpha \in \mathbb{T}$, set $D^{\alpha}:=D_{-\log r, N}$ and $K^{\alpha}:=$ $K_{-\log r, N}$ and $\chi^{\alpha}(t):=u(t)-i \log r, t \in D^{\alpha}$, where $u$ is a function of $t$ satisfying equation (11.4) with $s:=-\log r$. Consequently, using Lemma 10.1 we can show that $\left(K^{\alpha}\right)_{\alpha \in \mathbb{T}}$ is an interpretation of the geometric intersection $T \wedge\left[\mathfrak{C}_{r}\right]$ on $\mathbb{B}_{r^{1 / N}|\log r|^{-3 / N}}$ with mesh 1 .

It remains us to show that the above interpretation is coherent. We can check this using Lemma 7.9 and the fact that the mesh of the interpretation is 1.

\section{MASS OF $T \wedge\left[\mathfrak{C}_{r, N}\right]$ OUTSIDE THE CORONA $\mathbb{A}_{r, N}$}

The objective of this section is to establish Proposition 9.1. We start with the following simple lemma.

Lemma 12.1. Let $0<s<r<\infty$. Let $h$ be a positive harmonic function on the disc $\mathbb{D}_{r}$. Then there is a constant $c>0$ depending only on the quotient $s / r$ such that for $x_{1}, x_{2} \in \overline{\mathbb{D}}_{s}$,

$$
\left|h\left(x_{1}\right)-h\left(x_{2}\right)\right| \leq c r^{-3}\left|x_{1}-x_{2}\right| \int_{\mathbb{D}_{r}} h(z) d \operatorname{Leb}(z),
$$

where $d$ Leb is the Lebesgue measure in $\mathbb{C}$.

Proof. Using a continuity argument we may assume that $h$ is continuous on $\overline{\mathbb{D}}_{r}$. By Poisson integral formula, we have

$$
h(x)=\frac{1}{2 \pi r} \int_{\partial \mathbb{D}_{r}} \frac{r^{2}-|x|^{2}}{|x-y|^{2}} h(y) d \sigma(y) \quad \text { for } \quad x \in \mathbb{D}_{r}, y \in \partial \mathbb{D}_{r},
$$

where $d \sigma(y)$ is the Lebesgue measure on $\partial \mathbb{D}_{r}$. We infer from this formula that

$$
\begin{aligned}
\left|h\left(x_{1}\right)-h\left(x_{2}\right)\right| \leq c \frac{\left|x_{1}-x_{2}\right|}{r^{2}} \int_{\partial \mathbb{D}_{r}} h(y) d \sigma(y) & =\frac{2 \pi c\left|x_{1}-x_{2}\right| h(0)}{r} \\
& =\frac{2 c\left|x_{1}-x_{2}\right|}{r^{3}} \int_{\mathbb{D}_{r}} h(z) d \operatorname{Leb}(z),
\end{aligned}
$$


where the equalities hold by the mean-property. Hence, the lemma follows.

Now we are in the position to prove the first part of Proposition 9.1.

End of the proof of estimate (9.1) in Proposition 9.1. Let $N \in \mathbb{N}$ and let $r_{N}$ be given by Proposition 8.1. Consider the compact set

$$
Y:=\mathfrak{C}_{r, N} \cap\left(X \backslash \mathbb{D}^{2}\right) .
$$

By Proposition 8.1 (i) and (ii), we have that $Y \cap E=\varnothing$. We use the finite cover $\mathscr{U}$ of $X$ by singular flow boxes $\left(\mathbb{U}_{e}\right)_{e \in E}$ and regular flow boxes $\left(\mathbb{U}_{p}\right)_{p \in P}$ introduced in Subsection 2.2. We may assume without loss of generality that

$$
Y \subset \bigcup_{p \in P} \mathbb{U}_{p}
$$

Putting (8.6) and (8.8) and (12.1) together, we use an argument of local complex geometry to express the intersection points of the algebraic curves $\mathfrak{C}_{r, N}$ and $\mathfrak{C}_{0, N}$ with a plaque of $\mathbb{U}_{p}$ as the roots of some holomorphic functions defined on some open subset of $\mathbb{U}_{p}$. Consequently, by shrinking $r_{N}$ if necessary, we may find a constant $0<\delta=\delta_{N}<1$ such that for every $x \in \mathfrak{C}_{0, N} \cap\left(X \backslash \mathbb{D}^{2}\right)$ and every $0<r<r_{N}$, there is exactly one point $\tau(x) \in \mathfrak{C}_{r, N}$ such that $x$ and $\tau(x)$ are on the same plaque $\mathbb{V}_{x}$ of at least one of the regular flow boxes $\left(\mathbb{U}_{p}\right)_{p \in P}$ and that

$$
\operatorname{dist}(x, \tau(x)) \leq c r^{\delta} \quad \text { for some constant } \quad c>1 \text { independent of } x \text {. }
$$

In fact, $\delta$ is the reciprocal of the multiplicity of the intersection of $\mathfrak{C}_{0, N}$ and $\mathbb{V}_{x}$ at $x$.

By shrinking the union $\cup_{x \in \mathfrak{C}_{0, N}} \mathbb{V}_{x}$, we may find an open neighborhood $\mathbb{V}$ of $\left(X \backslash \mathbb{D}^{2}\right) \cap \mathfrak{C}$, where $\mathfrak{C}$ is the closure of $\bigcup_{0 \leq r<r_{N}} \mathfrak{C}_{r, N}$. Therefore, by Proposition 2.6 we have the following integral representation of $T$ in $\mathbb{V}$ :

$$
T=\int h_{x}\left[\mathbb{V}_{x}\right] d \nu(x),
$$

where, for each $x \in \mathfrak{C}_{0, N} \cap\left(X \backslash \mathbb{D}^{2}\right), h_{x}$ denotes the positive harmonic function associated to the current $T$ on the plaque $\mathbb{V}_{x}$, and $\nu$ is a positive Radon measure on $\mathfrak{C}_{r, N} \cap\left(X \backslash \mathbb{D}^{2}\right)$.

On the other hand, by Part 1) of Lemma 2.4, we have that

$$
c^{-1} \leq \eta(x) \leq c, \quad x \in \bigcup_{p \in P} \mathbb{U}_{p} \quad \text { for some constant } \quad c>1 .
$$

This, combined with (12.2) and (12.1), implies that $\operatorname{dist}_{P}(x, \tau(x)) \leq c r^{\delta}$ and that the diameter of the plaque $\mathbb{V}_{x}$ with respect to the Poincaré metric $g_{P}$ is $\approx 1$. Applying Lemma 12.1 to the disc $\mathbb{D}_{r}$ for $r \approx 1$, we get a constant $c>1$ such that

$$
\left|h_{x}(x)-h_{x}(\tau(x))\right| \lesssim \operatorname{dist}_{P}(x, \tau(x)) \int_{\mathbb{V}_{x}} h_{x}(y) g_{P}(y) \lesssim r^{\delta} \int_{\mathbb{V}_{x}} h_{x}(y) g_{P}(y) .
$$


Integrating both sides with respect to $d \nu(x)$, we obtain that

$$
\int_{x \in \mathfrak{C}_{0, N} \cap\left(X \backslash \mathbb{D}^{2}\right)}\left|h_{x}(x)-h_{x}(\tau(x))\right| d \nu(x) \lesssim r^{\delta}\left\|T \wedge g_{P}\right\|_{\mathbb{V}} \lesssim r^{\delta},
$$

where the last inequality holds because $\left\|T \wedge g_{P}\right\|_{X}$ is finite by [10, Proposition 4.2] (this corresponds to (1.2) in the case $\delta=1$ ). Since we know by using (12.1) and Proposition 7.1 that the left hand side is bigger than

$$
\left|\left\|T \wedge\left[\mathfrak{C}_{r, N}\right]\right\|_{X \backslash \mathbb{D}^{2}}-\left\|T \wedge\left[\mathfrak{C}_{0, N}\right]\right\|_{X \backslash \mathbb{D}^{2}}\right|,
$$

the desired estimate follows.

Remark 12.2. Estimate (9.1) in Proposition 9.1 still holds if we replace $X$ and $\mathbb{D}^{2}$ by the bidiscs $\mathbb{D}^{2}$ and $(s \mathbb{D})^{2}$ respectively for any $0<s<1$, i.e., there are constants $0<\delta=\delta_{N}<1$ and $c=c_{s, N}>1$ such that

$$
\left|\left\|T \wedge\left[\mathfrak{C}_{r, N}\right]\right\|_{\mathbb{D}^{2} \backslash(s \mathbb{D})^{2}}-\left\|T \wedge\left[\mathfrak{C}_{0, N}\right]\right\|_{\mathbb{D}^{2} \backslash(s \mathbb{\mathbb { D }})^{2}}\right| \leq c r^{\delta} \quad \text { for } \quad 0<r<\min \left\{s, r_{N}\right\} .
$$

The last two parts of Proposition 9.1 concern balls in a singular flow box around a singular point $\bar{x} \in E$. In what follows, we may assume without loss of generality that $\mathscr{F}$ is the foliation on $\mathbb{D}^{2}$ associated to the vector field $F$ introduced in Section 5 and that $\bar{x}=0 \in \mathbb{D}^{2}$. Moreover, let $c_{0}$ be the constant $c>1$ given by Lemma 10.5 (ii).

Definition 12.3. Given a point $x_{0}=\left(z_{0}, w_{0}\right) \in(\mathbb{D} \backslash\{0\})^{2}$ and a number $0<\rho<$ $1 / 2$, a cell with center $x_{0}$ and radius $\rho$ is the set $\operatorname{Cell}\left(x_{0}, \rho\right)$ given by

$\left\{x=(z, w) \in(\mathbb{D} \backslash\{0\})^{2}: \max \left\{\left|1-z / z_{0}\right|,\left|1-z_{0} / z\right|,\left|1-w / w_{0}\right|,\left|1-w_{0} / w\right|\right\}<\rho\right\}$.

Note that for $x \in \operatorname{Cell}\left(x_{0}, \rho\right)$,

$$
(1+\rho)^{-1}\left\|x_{0}\right\| \leq\|x\| \leq(1+\rho)\left\|x_{0}\right\| .
$$

We fix a number $0<\rho_{0}<1 / 2$ so that the following two conditions (i)-(ii) are satisfied:

(i) For every $0<\rho \leq \rho_{0}$ and every point $x_{0}=\left(z_{0}, w_{0}\right) \in(\mathbb{D} \backslash\{0\})^{2}$, Cell $\left(x_{0}, \rho\right)$ is a flow box with the transversals

$\mathbb{T}_{x_{0}}:=\left\{\left(z_{0}, w\right) \in \operatorname{Cell}\left(x_{0}, \rho\right): w \in \mathbb{C}\right\} \quad$ and $\quad \mathbb{T}_{x_{0}}^{\prime}:=\left\{\left(z, w_{0}\right) \in \operatorname{Cell}\left(x_{0}, \rho\right): z \in \mathbb{C}\right\}$.

We often identify $\mathbb{T}_{x_{0}}$ and $\mathbb{T}_{x_{0}}^{\prime}$ with its projection on second first and its first components respectively, that is, with the set $\left\{w \in \mathbb{D}:\left|1-w / w_{0}\right|<\rho\right.$ and $\mid 1-$ $\left.w_{0} / w \mid<\rho\right\}$ and $\left\{z \in \mathbb{D}:\left|1-z / z_{0}\right|<\rho\right.$ and $\left.\left|1-z_{0} / z\right|<\rho\right\}$ respectively. The plaque of $\mathbb{V}:=\operatorname{Cell}\left(x_{0}, \rho\right)$ passing through $\alpha=\left(z_{0}, w\right) \in \mathbb{T}_{x_{0}}$ is denoted by $\mathbb{V}_{\alpha}$.

(ii) All points in $\mathbb{V}_{\alpha}$ are compatible with each other in the sense of Definition 10.4, in particular with $\alpha$, for all $\alpha \in \mathbb{T}_{x_{0}}$.

Finally, set $\rho_{1}:=1 / 4 c_{0}^{-2} \rho_{0}$.

The following result illustrates the usefulness of the constants $\rho_{0}$ and $\rho_{1}$ given in Definition 12.3, 
Proposition 12.4. Let $\mathbb{U}$ (resp. $\mathbb{V}$ ) be the cell with center $x_{0} \in(\mathbb{D} \backslash\{0\})^{2}$ and radius $\rho_{0}$ (resp. radius $\rho_{1}$ ). Let $\mathbb{T}_{x_{0}}$ be a transversal of $\mathbb{V}$ as in Definition 12.3 Let $\mathfrak{D}_{1}, \mathfrak{D}_{2}$ be two analytic curves in $\mathbb{U}$ such that for every $\alpha \in \mathbb{T}_{x_{0}}$ and $j=1,2$, $\mathfrak{D}_{j}$ intersects the plaque $\mathbb{V}_{\alpha}$ at a unique point $\alpha_{j}$. Then there is a constant $c>0$ independent of $x_{0}$ such that for every harmonic current $T$ tangent to the foliation, we have that

$$
\left\|\left[\mathfrak{D}_{1}\right] \wedge T-\left[\mathfrak{D}_{2}\right] \wedge T\right\|_{\mathbb{V}} \leq c \frac{\left(\log ^{*}\left|x_{0}\right|\right)^{2}\left\|T \wedge g_{P}\right\|_{\mathbb{U}}}{\left\|x_{0}\right\|} \sup _{\alpha \in \mathbb{T}_{x_{0}}}\left\|\alpha_{1}-\alpha_{2}\right\| .
$$

Proof. By Proposition 2.6 we have the following integral representation of $T$ in $\mathbb{V}:$

$$
T=\int h_{\alpha}\left[\mathbb{V}_{\alpha}\right] d \nu(\alpha),
$$

where, for each $\alpha \in \mathbb{T}_{x_{0}}, h_{\alpha}$ denotes the positive harmonic function associated to the current $T$ on the plaque $\mathbb{V}_{\alpha}$.

Let $\alpha \in \mathbb{T}_{x_{0}}$. Since we know by Definition 12.3 that $\|\alpha\| \approx\left\|x_{0}\right\|$ and that the points in $\mathbb{V}_{\alpha}$ are compatible with each other, it follows from Lemma 10.5 (ii) and the choice of $\rho_{0}, \rho_{1}$ that there are two constants $0<c^{\prime}<c^{\prime \prime}$ (independent of $\alpha$ and $x_{0}$ ) and constants $c_{\alpha}, c_{\alpha}^{\prime} \in\left[c^{\prime}, c^{\prime \prime}\right]$ such that

$$
\phi_{\alpha}\left(\mathbb{D}_{c_{\alpha}\left(\log ^{*}\left\|x_{0}\right\|\right)^{-1}}\right) \subset \mathbb{V}_{\alpha} \subset \phi_{\alpha}\left(\mathbb{D}_{c_{\alpha}^{\prime}\left(\log ^{*}\left\|x_{0}\right\|\right)^{-1}}\right) \subset \phi_{\alpha}\left(\mathbb{D}_{2 c_{\alpha}^{\prime}\left(\log ^{*}\left\|x_{0}\right\|\right)^{-1}}\right) \subset \mathbb{U}_{\alpha} .
$$

Therefore, applying Lemma 12.1 to the disc $\mathbb{D}_{r}$ for $r:=2 c_{\alpha}^{\prime}\left(\log ^{*}\left\|x_{0}\right\|\right)^{-1}$, we get a constant $c>1$ such that for $x_{1}, x_{2} \in \mathbb{V}_{\alpha}$, we have that

$$
\left|h_{\alpha}\left(x_{1}\right)-h_{\alpha}\left(x_{2}\right)\right| \leq c\left\|x_{0}\right\|^{-1}\left(\log ^{*}\left\|x_{0}\right\|\right)^{2}\left|x_{1}-x_{2}\right| \int_{\mathbb{U}_{\alpha}} h_{\alpha} g_{P},
$$

where $g_{P}$ is, as usual, the leafwise Poincaré metric restricted to $\mathbb{U}_{\alpha} \subset L_{\alpha}$. Applying the last inequality to $x_{1}=\alpha_{1}$ and $x_{2}=\alpha_{2}$ and integrating both sides with respect to $d \nu(\alpha)$ and using the above integral representation of $T$, we obtain that

$$
\int_{\alpha \in \mathbb{T}_{x_{0}}}\left|h_{\alpha}\left(\alpha_{1}\right)-h_{\alpha}\left(\alpha_{2}\right)\right| d \nu(\alpha) \leq c \frac{\left(\log ^{*}\left|x_{0}\right|\right)^{2}\left\|T \wedge g_{P}\right\|_{\mathbb{U}}}{\left\|x_{0}\right\|} \sup _{\alpha \in \mathbb{T}_{x_{0}}}\left\|\alpha_{1}-\alpha_{2}\right\| .
$$

Applying Proposition 7.1, we see that the left hand side is bigger than $\|\left[\mathfrak{D}_{1}\right] \wedge$ $T-\left[\mathfrak{D}_{2}\right] \wedge T \|_{\mathbb{V}}$, and hence the proposition follows.

End of the proof of estimate (9.2) in Proposition 9.1, Fix $N \in \mathbb{N}$ large enough and let $0<r_{N}<1$ be the constant given by Lemma 10.7. Set $s:=r_{N}$. By Remark 12.2 we can reduce estimate (9.2) to the following one:

$$
\left|\left\|T \wedge\left[\mathfrak{C}_{r, N}\right]\right\|_{(s \mathbb{D})^{2} \backslash \mathbb{B}_{r^{1 / N}|\log r|^{3 / N}}}-\left\|T \wedge\left[\mathfrak{C}_{0, N}\right]\right\|_{(s \mathbb{D})^{2} \backslash \mathbb{B}_{r^{1 / N}|\log r|^{3 / N}}}\right| \leq c|\log r|^{-1}
$$


where $c$ is a constant which depends only on $N$. Fix $\rho_{2}$ with $0<\rho_{2} \ll \rho_{0}$ and $2 \pi \rho_{2}^{-1} \in \mathbb{N}$. Consider the countable set

$$
\begin{aligned}
\mathbb{X}:=\left\{x=(z, w) \in(s \mathbb{D})^{2}: \quad|z|,|w|\right. & \in\left\{s\left(1-\rho_{2}\right)^{p}: p \in \mathbb{N}\right\} \\
& \text { and } \left.\arg z, \arg w \in\left\{0, \rho_{2}, 2 \rho_{2} \ldots, 2 \pi\right\}\right\} .
\end{aligned}
$$

Consider the family $\mathscr{C}$ of cells $\operatorname{Cell}\left(x, \rho_{0}\right)$, where $x \in \mathbb{X}$. We see easily that when $\rho_{2}$ is small enough and when a constant $0<\rho_{3} \ll \rho_{1}$ is small enough, the following property holds:

Property (i). For every point $x \in(s \mathbb{D} \backslash\{0\})^{2}$, there exists at least one cell $C \in \mathscr{C}$ such that $\operatorname{Cell}\left(x, \rho_{3}\right) \subset C$.

In particular, Property (i) implies that

$$
(s \mathbb{D})^{2} \backslash(\{z=0\} \cup\{w=0\})=\bigcup_{x \in \mathbb{X}} \operatorname{Cell}\left(x, \rho_{0}\right) .
$$

Moreover, we can check that the following property also holds.

Property (ii). There is $K \in \mathbb{N}$ such that each point in $(s \mathbb{D} \backslash\{0\})^{2}$ belongs to at most $K$ cells in the family $\mathscr{C}$.

Let $0<r<r_{N}$ be arbitrary. Combing Lemma 10.6 and Lemma10.7, we see that for every $\alpha \in \mathbb{T}$ and $k \in \mathbb{N}$ with $\xi_{0, N, \alpha, k} \in(s \mathbb{D})^{2} \backslash \mathbb{B}_{r^{1 / N}|\log r|^{3 / N}}$,

$$
\operatorname{dist}_{C}\left(\xi_{r, N, \alpha, k}, \xi_{0, N, \alpha, k}\right) \lesssim|\log r|^{-3} \text { and } \operatorname{dist}_{C}\left(\xi_{N, \alpha, k}, \xi_{0, N, \alpha, k}\right) \approx N^{-1}
$$

Fix $\alpha_{0} \in \mathbb{T}$. Suppose that there is $k \in \mathbb{N}$ such that $\xi_{0, N, \alpha_{0}, k} \in(s \mathbb{D})^{2} \backslash \mathbb{B}_{r^{1 / N}|\log r|^{3 / N}}$. By Property (i), we may find a cell $C=C_{k} \subset \mathscr{C}$ such that $C^{\prime}:=\operatorname{Cell}\left(\xi_{0, N, \alpha_{0}, k}, \rho_{3}\right) \subset$ $C$. Set $\rho_{4}:=1 / 4 c_{0}^{-2} \rho_{3}$, where the constant $c_{0}>1$ is introduced just before Definition 12.3. Next, using (12.4) we can check that there are constants $c^{\prime}>1$ and $0<\rho_{5} \ll 1$ depending only on $\rho_{0}, \rho_{1}, \rho_{2}, \rho_{3}$ (in particular, they are independent of $r$ and $N$ ) with the following property:

There is an open ball $W_{\alpha_{0}}$ with center $\alpha_{0} \in \mathbb{T}$ and radius $\rho_{5}$ in $\mathbb{T}$, where $\mathbb{T}$ is defined in (5.4), and an interval $S_{r, \alpha_{0}} \subset \mathbb{N}$ such that

- $c^{\prime-1} N \leq \# S_{r, \alpha_{0}} \leq c^{\prime} N$, where \# denotes the cardinality;

- for every $k \in S_{r, \alpha_{0}}$ and $\alpha \in W_{\alpha_{0}}$, all three points $\xi_{N, \alpha, k}, \xi_{0, N, \alpha, k}, \xi_{r, N, \alpha, k}$ not only belong to the bidisc $(s \mathbb{D})^{2}$, but also belong to the cell $C^{\prime \prime}:=\operatorname{Cell}\left(\xi_{0, N, \alpha_{0}, k}, \rho_{4}\right)$.

Let $\mathbb{T}_{C^{\prime \prime}}$ be a transversal of $C^{\prime \prime}$ in the sense of Definition 12.3. For every $W \subset \mathbb{T}$, let $\widehat{L}_{W}:=\bigcup_{\alpha \in W} \widehat{L}_{\alpha}$. Set

$$
\mathbb{T}_{C^{\prime \prime}, \alpha_{0}}:=\mathbb{T}_{C^{\prime \prime}} \cap \widehat{L}_{W_{\alpha_{0}}} .
$$

This is a nonempty open subset of $\mathbb{T}_{C^{\prime \prime}}$. This, combined with (12.4), allows us to apply Proposition 12.4 to two algebraic curves $\mathfrak{C}_{0, N}$ and $\mathfrak{C}_{r, N}$ in the cells $C^{\prime}$ and $C^{\prime \prime}$. The only change is that we use the constants $\rho_{3}, \rho_{4}$ instead of $\rho_{0}, \rho_{1}$. 
Consequently, we get a constant $c^{\prime}>0$ such that

$$
\begin{aligned}
& \int_{\alpha \in \mathbb{T}_{C^{\prime \prime}, \alpha_{0}}} \mid h_{\alpha}\left(\xi_{0, N, \alpha, k}\right)-h_{\alpha}\left(\xi_{r, N, \alpha, k}\right) \mid d \nu(\alpha) \\
& \lesssim c^{\prime} \frac{\left.|\log | \xi_{0, N, \alpha_{0}, k_{0}}\right|^{2}\left\|T \wedge g_{P}\right\|_{C^{\prime}}}{\left\|\xi_{0, N, \alpha_{0}, k_{0}}\right\|} \cdot \sup _{\alpha \in \mathbb{T}}\left\|\xi_{0, N, \alpha, k}-\xi_{r, N, \alpha, k}\right\| .
\end{aligned}
$$

Using the first estimate in (12.4) and the inequality $\left|\xi_{0, N, \alpha_{0}, k_{0}}\right| \geq r^{1 / N}|\log r|^{3 / N}$, the right hand side is bounded from above by a constant times $|\log r|^{-1} \| T \wedge$ $g_{P} \|_{C}$. On the other hand, the left hand side is bounded from below by $\mid\left[\mathfrak{C}_{0, N}\right] \wedge$ $T-\left[\mathfrak{C}_{r, N}\right] \wedge T \|_{C \cap \widehat{L}_{W_{\alpha_{0}}}}$. Hence, for $C=C_{k}$ we have that

$$
\left|\left[\mathfrak{C}_{0, N}\right] \wedge T-\left[\mathfrak{C}_{r, N}\right] \wedge T\left\|_{C^{\prime \prime} \cap \widehat{L}_{W_{\alpha_{0}}}} \lesssim|\log r|^{-1}\right\| T \wedge g_{P} \|_{C}\right.
$$

Summing up the last inequality over all $k \in S_{r, \alpha_{0}}$, and using Property (ii) above, we get that

$$
\left|\left[\mathfrak{C}_{0, N}\right] \wedge T-\left[\mathfrak{C}_{r, N}\right] \wedge T\left\|_{\widehat{L}_{W_{\alpha_{0}}}} \lesssim|\log r|^{-1}\right\| T \wedge g_{P} \|_{X}\right.
$$

A compactness argument shows that we can cover $\overline{\mathbb{T}}$ by a finite number of open sets $W_{\alpha_{0}}$. Applying the above estimate to each element of this cover and summing up the obtained estimates, (12.3) follows and we are done.

End of the proof of estimate (9.3) in Proposition 9.1. We argue as in the above proof of estimate (9.2) in Proposition 9.1 using Lemma 10.8 instead of Lemma 10.7. We only point out here the necessary modification. Fix $\alpha_{0} \in \mathbb{T}$. Suppose that there is $k \in \mathbb{N}$ such that $\xi_{r, \alpha_{0}, k} \in \mathbb{B}_{r^{1 / N}|\log r|^{-3 / N}}$. By Property (i) in the previous proof, we may find a cell $C=C_{k} \subset \mathscr{C}$ such that $\operatorname{Cell}\left(\xi_{r, \alpha_{0}, k}, \rho_{3}\right) \subset$ $C$. Thus, there is an open ball $W_{\alpha_{0}}$ with center $\alpha_{0}$ and radius $\rho_{5}$ in $\mathbb{T}$ and an interval $S_{r, \alpha_{0}} \subset \mathbb{N}$ such that $c^{-1} N \leq \# S_{r, \alpha_{0}} \leq c^{\prime} N$ points, and that for every $k \in S_{r, \alpha_{0}}$ and $\alpha \in W_{\alpha_{0}}$, the two points $\xi_{r, \alpha, k}$ and $\xi_{r, N, \alpha, k}$ belong to the cell $C^{\prime \prime}:=\operatorname{Cell}\left(\xi_{r, \alpha_{0}, k}, \rho_{4}\right)$.

Next, using Lemma 10.8 instead of the first estimate in (12.4), we conclude the proof as in the previous one.

\section{MASS OF $T \wedge\left[\mathfrak{C}_{r}\right], T \wedge\left[\mathfrak{C}_{0, N}\right], T \wedge\left[\mathfrak{C}_{r, N}\right]$ ON THE CORONA $\mathbb{A}_{r, N}$}

The objective of this section is to establish Proposition 9.2. Recall that for every $s>0$, the function $K_{s}: \mathbb{R} \rightarrow \mathbb{R}^{+}$is given by (6.3).

13.1. Mass of $T \wedge\left[\mathfrak{C}_{r}\right]$ on $\mathbb{A}_{r, N}$. In order to prove the first inequality of this proposition, we consider, for each $s>1$ and $N \in \mathbb{N} \backslash\{0\}$, the following domain in $\mathbb{R}$ :

$$
D_{s, N}:=\left\{t \in \mathbb{R}^{+}: N^{-1}(s-3 \log s) \leq t \leq N^{-1}(s+3 \log s)\right\}
$$


and the function $K_{s, N}^{(1)}: \mathbb{R} \rightarrow \mathbb{R}^{+}$given by

$$
K_{s, N}^{(1)}(y):=\int_{D_{s, N}} \frac{V}{V^{2}+(y-U)^{2}} d t, \quad y \in \mathbb{R},
$$

Here $U, V$ are functions of the variable $t$ and the parameter $s$ which satisfy the following system of equations (see (5.6), (5.2) and (5.3)):

$$
U+i V=(u+i s)^{\gamma} \quad \text { and } \quad t=b u+a s .
$$

It is worthy comparing the domain $D_{s, N}$ (resp. the function $K_{s, N}^{(1)}$ ) with the domains $D_{s}$ (resp. the function $\widetilde{K}_{s}$ ) given in (11.1).

Lemma 13.1. For every $0<r<1 / 2$,

$$
\left\|T \wedge\left[\mathfrak{C}_{r}\right]\right\|_{\mathbb{A}_{r, N}} \approx \int_{\alpha \in \mathbb{T}}\left(\int_{-\infty}^{\infty} K_{-\log r, N}^{(1)}(y) \tilde{H}_{\alpha}(y) d y\right) d \nu(\alpha) .
$$

Proof. Using (10.1), (5.1) and (5.3), we see that $\xi_{r, \alpha, k} \in \mathbb{A}_{r, N}$ if and only if

$$
\begin{aligned}
\frac{1}{2 \pi b}\left(\left(N^{-1}-a\right)(-\log r)\right. & -3 \log (-\log r)+\log |\alpha|) \leq k \\
\leq & \frac{1}{2 \pi b}\left(\left(N^{-1}-a\right)(-\log r)+3 \log (-\log r)+\log |\alpha|\right) .
\end{aligned}
$$

Let $\mathbb{Z}_{r, N, \alpha}^{1}$ be the set of all integers $k$ satisfying the last inequalities. Observe that

$$
\left\|T \wedge\left[\mathfrak{C}_{r}\right]\right\|_{\mathbb{A}_{r, N}}=\int_{\alpha \in \mathbb{T}}\left(\sum_{k \in \mathbb{Z}_{r, N, \alpha}^{1}} h_{\alpha}\left(\xi_{r, \alpha, k}\right)\right) d \nu(\alpha) .
$$

For $\alpha \in \mathbb{T}$ let $D^{\alpha}:=D_{-\log r, N}, t_{r, \alpha, k}:=b u_{\alpha, k}+a v_{r}, k \in \mathbb{Z}$. Then $k \in \mathbb{Z}_{r, N, \alpha}$ if and only if $t_{r, \alpha, k} \in D^{\alpha}$. Moreover, consider the function $\chi^{\alpha}: D^{\alpha} \rightarrow \mathbb{C}$ given by

$$
\chi^{\alpha}(t):=u+i v_{r}, \quad \text { where } \quad v_{r}=-\log r \quad \text { and } \quad t=b u+a v_{r} .
$$

Let $m=1$ and choose $\rho>1$ large enough. Using Harnack's inequality, we see that the assumption of Part 1) of Proposition 7.7 is also fulfilled. In other words, by Definition 7.8 we obtain an interpretation $\left(K^{\alpha}\right)_{\alpha \in \mathbb{T}}$ of the geometric intersection $T \wedge\left[\mathfrak{C}_{r}\right]$ on $\mathbb{A}_{r, N}$ with mesh $m=1$. Consequently, applying Part 1) of Proposition 7.7 the lemma follows.

Lemma 13.2. There is a constant $c>1$ such that for all $y \in \mathbb{R}, s>1$ and $N \in \mathbb{N} \backslash\{0\}$

$$
K_{s, N}^{(1)}(y) \leq c s^{-1}(\log s) K_{s}(y) .
$$

Proof. We follow the method of proof of Lemma 11.1. Let $c_{2}, c_{3}$ be the constants with $c_{3}>c_{2}>1$ given by Lemma 7.9 . We consider three cases.

Case 1: $s \geq c_{2}(1+|y|)^{1 / \gamma}$. 
By Part 2) of Lemma 7.9 and by formula (13.2), we have that

$$
K_{s, N}^{(1)}(y) \approx \int_{t=s / N-3(\log s) / N}^{t=s / N+3(\log s) / N} \frac{(s / N) d t}{s^{\gamma+1}} \approx N^{-2} s^{-\gamma} \log s .
$$

This, compared with formula (6.3), completes the proof of Case 1 .

Case 2: $c_{3}^{-1}(1+|y|)^{1 / \gamma} \leq s \leq c_{2}(1+|y|)^{1 / \gamma}$.

Applying Part 5) of Lemma 7.9, we get that

$$
K_{s, N}^{(1)}(y) \approx \int_{t=s / N-3(\log s) / N}^{t=s / N+3(\log s) / N} \frac{(1+|y|)^{1 / \gamma-1} t d t}{t^{2}+(s-\rho(y, t))^{2}}
$$

where $\rho(y, t)$ satisfies $c_{2}^{-1}(1+|y|)^{1 / \gamma} \leq \rho(y, t) \leq c_{2}(1+|y|)^{1 / \gamma}$. A straightforward computation shows that the right hand side is $\approx N^{-1} s^{-1} \log s(1+|y|)^{1 / \gamma-1}$. Comparing this with formula (6.3), the proof of Case 2 is complete.

Case 3: $s \leq c_{3}^{-1}(1+|y|)^{1 / \gamma}$.

Applying Part 1) and Part 3) of Lemma 7.9, we get that

$$
K_{s, N}^{(1)}(y) \approx \int_{t=s / N-3(\log s) / N}^{t=s / N+3(\log s) / N} \frac{t^{\gamma-1} s d t}{(1+|y|)^{2}} \approx N^{-\gamma} s^{\gamma} \log s(1+|y|)^{-2} .
$$

This, compared with formula (6.3), allows us to conclude the proof of the last case.

End of the proof of inequality (9.5) in Proposition 9.2. Applying Lemma 13.1 and then Lemma 13.2, we see that for every $0<r<1 / 2$,

$$
\begin{aligned}
\left\|T \wedge\left[\mathfrak{C}_{r}\right]\right\|_{\mathbb{A}_{r, N}} & \approx \int_{\alpha \in \mathbb{T}}\left(\int_{-\infty}^{\infty} K_{-\log r, N}^{(1)}(y) \tilde{H}_{\alpha}(y) d y\right) d \nu(\alpha) \\
& \lesssim N^{-1}(-\log r)^{-1} \log (-\log r) \int_{\alpha \in \mathbb{T}}\left(\int_{-\infty}^{\infty} K_{-\log r}(y) \tilde{H}_{\alpha}(y) d y\right) d \nu(\alpha) .
\end{aligned}
$$

By Lemma 6.1 and identity (6.2), the integral in the last line is uniformly bounded in $r$. The proof is thereby completed.

13.2. Mass of $T \wedge\left[\mathfrak{C}_{0, N}\right]$ on $\mathbb{A}_{r, N}$. The next part of this section is devoted to the proof of the second inequality of Proposition 9.2. We consider, for each $s>1$ and $N \in \mathbb{N} \backslash\{0\}$, the function $K_{s, N}^{(2)}: \mathbb{R} \rightarrow \mathbb{R}^{+}$given by

$$
K_{s, N}^{(2)}(y):=N^{2} \int_{D_{s, N}} \frac{V}{V^{2}+(y-U)^{2}} d t, \quad y \in \mathbb{R} .
$$

Here the domain $D_{s, N}$ is given in (13.1), and $U, V$ are functions of the variable $t$ which satisfy the following system of equations (see (5.6), (5.2) and (5.3)):

$$
U+i V=(u+i v)^{\gamma} \text { and } t=b u+a v \text { and } v=N t+\log |d|,
$$

where $d:=-a_{N}$ (see (8.7) and Lemma 10.6 for $a_{N}$ ). 
Lemma 13.3. For every $0<r<1 / 2$,

$$
\left\|T \wedge\left[\mathfrak{C}_{0, N}\right]\right\|_{\mathbb{A}_{r, N}} \approx \int_{\alpha \in \mathbb{T}}\left(\int_{-\infty}^{\infty} K_{-\log r, N}^{(2)}(y) d y\right) d \nu(\alpha) .
$$

Proof. Using (10.2), (10.3), (10.4), (5.1), (15.2) and (5.3), we see that $\xi_{N, \alpha, k} \in$ $\mathbb{A}_{r, N}$ if and only if

$$
(-\log r)-3 \log (-\log r) \leq t_{N, \alpha, k} \leq(-\log r)+3 \log (-\log r) .
$$

Let $\mathbb{Z}_{N, \alpha}^{1}$ be the set of all integers $k$ satisfying the last inequalities. Observe that

$$
\begin{aligned}
\left\|T \wedge\left[\mathfrak{C}_{N}^{d}\right]\right\|_{\mathbb{A}_{r, N}} & =\int_{\alpha \in \mathbb{T}}\left(\sum_{k \in \mathbb{Z}_{N, \alpha}^{1}} h_{\alpha}\left(\xi_{N, \alpha, k}\right)\right) d \nu(\alpha) \\
\left\|T \wedge\left[\mathfrak{C}_{0, N}\right]\right\|_{\mathbb{A}_{r, N}} & =\int_{\alpha \in \mathbb{T}}\left(\sum_{k \in \mathbb{Z}_{N, \alpha}^{1}} h_{\alpha}\left(\xi_{0, N, \alpha, k}\right)\right) d \nu(\alpha) .
\end{aligned}
$$

On the other hand, by Lemma 10.6 we know that $\xi_{N, \alpha, k}$ and $\xi_{0, N, \alpha, k}$ are compatible for $k \in \mathbb{Z}_{N, \alpha}^{1}$. Hence, by Harnack's inequality, there is a constant $c>1$ such that

$$
c^{-1} h_{\alpha}\left(\xi_{N, \alpha, k}\right) \leq h_{\alpha}\left(\xi_{0, N, \alpha, k}\right) \leq c h_{\alpha}\left(\xi_{N, \alpha, k}\right)
$$

This, combined with the above equalities, implies that

$$
\left\|T \wedge\left[\mathfrak{C}_{0, N}\right]\right\|_{\mathbb{A}_{r, N}} \approx \int_{\alpha \in \mathbb{T}}\left(\sum_{k \in \mathbb{Z}_{N, \alpha}^{1}} h_{\alpha}\left(\xi_{N, \alpha, k}\right)\right) d \nu(\alpha) .
$$

So we need to show that the right hand side in the last line is equivalent to the right hand side of the lemma. For $\alpha \in \mathbb{T}$ let $D^{\alpha}:=D_{-\log r, N}, t_{N, \alpha, k}:=$ $b u_{N, \alpha, k}+a v_{N, \alpha, k}, k \in \mathbb{Z}$. Then $k \in \mathbb{Z}_{N, \alpha}^{1}$ if and only if $t_{N, \alpha, k} \in D^{\alpha}$. Moreover, consider the function $\chi^{\alpha}: D^{\alpha} \rightarrow \mathbb{C}$ given by

$\chi^{\alpha}(t):=u_{N}(t)+i v_{N}(t), \quad$ where $\quad v_{N}(t)=N t+\log |d|$ and $t=b u_{N}(t)+a v_{N}(t)$.

By (10.4), (10.5) and (10.6), we choose $m=N^{-2}$. Moreover, take $\rho>1$ large enough. Using Harnack's inequality, we see that the assumption of Part 1) of Proposition 7.7 is fulfilled. Hence, we obtain an interpretation $\left(K^{\alpha}\right)_{\alpha \in \mathbb{T}}$, in the sense of Definition 7.8 , of the geometric intersection $T \wedge\left[\mathfrak{C}_{r}\right]$ on $\mathbb{A}_{r, N}$ with mesh $m=N^{-2}$. Consequently, applying Part 1) of Proposition 7.7 the lemma follows.

Lemma 13.4. There is a constant $c>1$ such that for all $y \in \mathbb{R}$ and $s>1$ and $N \in \mathbb{N} \backslash\{0\}$

$$
K_{s, N}^{(2)}(y) \leq c N^{2} s^{-1}(\log s) K_{s}(y) .
$$

Proof. We follow the method of proof of Lemma 11.1. Let $c_{2}, c_{3}$ be the constants with $c_{3}>c_{2}>1$ given by Lemma 7.9 . We consider three cases.

Case 1: $s \geq c_{2}(1+|y|)^{1 / \gamma}$. 
By Part 2) of Lemma 7.9 and by formula (13.3), we have that

$$
K_{s, N}^{(2)}(y) \approx N^{2} \int_{t=s / N-3(\log s) / N}^{t=s / N+3(\log s) / N} \frac{t d t}{(N t)^{\gamma+1}} \approx s^{-\gamma} \log s .
$$

This, compared with formula (6.3), completes the proof of Case 1 .

Case 2: $c_{3}^{-1}(1+|y|)^{1 / \gamma} \leq s \leq c_{2}(1+|y|)^{1 / \gamma}$.

Applying Part 5) of Lemma 7.9, we get that

$$
K_{s, N}^{(2)}(y) \approx N^{2} \int_{t=s / N-3(\log s) / N}^{t=s / N+3(\log s) / N} \frac{(1+|y|)^{1 / \gamma-1} t d t}{t^{2}+(N t-\rho(y, t))^{2}},
$$

where $\rho(y, t)$ satisfies $c_{2}^{-1}(1+|y|)^{1 / \gamma} \leq \rho(y, t) \leq c_{2}(1+|y|)^{1 / \gamma}$. A straightforward computation shows that the right hand side is $\approx N s^{-1} \log s(1+|y|)^{1 / \gamma-1}$. On the other hand, by formula (6.3) we have that $K_{s}(y) \approx(1+|y|)^{1 / \gamma-1}$. This completes the proof of Case 2 .

Case 3: $s \leq c_{3}^{-1}(1+|y|)^{1 / \gamma}$.

Applying Part 1) and Part 3) of Lemma 7.9, we get that

$$
K_{s, N}^{(2)}(y) \approx N^{2} \int_{t=s / N-3(\log s) / N}^{t=s / N+3(\log s) / N} \frac{t^{\gamma-1}(N t) d t}{(1+|y|)^{2}} \approx N^{2-\gamma} s^{\gamma} \log s(1+|y|)^{-2} .
$$

Since we know by formula $(6.3)$ that $K_{s}(y) \approx(1+|y|)^{1 / \gamma-1}$, the proof of the last case, and hence the lemma, is thereby completed.

End of the proof of inequality (9.6) in Proposition 9.2. Applying Lemma 13.3 and then Lemma 13.4, we see that for every $0<r<1 / 2$,

$$
\begin{aligned}
\left\|T \wedge\left[\mathfrak{C}_{0, N}\right]\right\|_{\mathbb{A}_{r, N}} & \approx \int_{\alpha \in \mathbb{T}}\left(\int_{-\infty}^{\infty} K_{-\log r, N}^{(2)}(y) \tilde{H}_{\alpha}(y) d y\right) d \nu(\alpha) \\
& \lesssim N(-\log r)^{-1} \log (-\log r) \int_{\alpha \in \mathbb{T}}\left(\int_{-\infty}^{\infty} K_{-\log r}(y) \tilde{H}_{\alpha}(y) d y\right) d \nu(\alpha) .
\end{aligned}
$$

By Lemma 6.1 and identity (6.2), the integral in the last line is uniformly bounded in $r$. The proof is thereby completed.

13.3. Mass of $T \wedge\left[\mathfrak{C}_{r, N}\right]$ on $\mathbb{A}_{r, N}$. The remainder of the section is devoted to the proof of inequality (9.7) in Proposition 9.2.

Let $0<\kappa=\kappa_{N} \ll 1$ be a very small constant whose exact value will be determined later on, and let $r_{N}>0$ be the constant satisfying both Lemmas 10.7 and 10.8, Write

$$
\left\|T \wedge\left[\mathfrak{C}_{r, N}\right]\right\|_{\mathbb{A}_{r, N}}=\left\|T \wedge\left[\mathfrak{C}_{r, N}\right]\right\|_{\mathbb{A}_{r, N} \cap \mathbb{B}_{\kappa r 1 / N}}+\left\|T \wedge\left[\mathfrak{C}_{r, N}\right]\right\|_{\mathbb{A}_{r, N} \backslash \mathbb{B}_{\kappa r} 1 / N}=: I+I I .
$$

Arguing as in the proof of Lemma 10.8 and replacing the ball $\mathbb{B}_{r^{1 / N}|\log r|^{-3 / N}}$ with $\mathbb{B}_{\kappa r^{1 / N}}$ and choosing $0<\kappa<1$ small enough, we obtain the following weaker result for every $0<r<r_{N}$ and $\alpha \in \mathbb{T}$ : The intersection of the curve $\mathfrak{C}_{r, N}$ with the Riemann surface $\widehat{L}_{\alpha}$ inside the ball $\mathbb{B}_{\kappa r^{1 / N}}$ can be enumerated 
as $\xi_{r, N, \alpha, k}$ such that $\xi_{r, N, \alpha, k}$ and $\xi_{r, \alpha, k}$ are compatible, where $k \in \mathbb{N}$ such that $\xi_{r, \alpha, k} \in \mathbb{B}_{\kappa r^{1 / N}}$. Consequently, we get that

$$
I=\int_{\alpha \in \mathbb{T}}\left(\sum h_{\alpha}\left(\xi_{r, N, \alpha, k}\right)\right) d \nu(\alpha),
$$

where the sum is taken over all $k \in \mathbb{N}$ such that $\zeta_{r, \alpha, k} \in \mathbb{A}_{r, N} \cap \mathbb{B}_{\kappa r^{1 / N}}$. Moreover, using that $\zeta_{r, N, \alpha, k}$ and $\zeta_{r, \alpha, k}$ are compatible, an application of Harnack's inequality gives that

$$
h_{\alpha}\left(\xi_{r, N, \alpha, k}\right) \leq c^{\prime} h_{\alpha}\left(\xi_{r, \alpha, k}\right) \quad \text { for some constant } \quad c^{\prime}>1 .
$$

Therefore, we infer that

$$
I \leq c^{\prime} \int_{\alpha \in \mathbb{T}}\left(\sum h_{\alpha}\left(\xi_{r, \alpha, k}\right)\right) d \nu(\alpha)=c^{\prime}\left\|T \wedge\left[\mathfrak{C}_{r}\right]\right\|_{\mathbb{A}_{r, N} \cap \mathbb{B}_{\kappa r^{1 / N}}} .
$$

The right hand side is bounded from above by $c^{\prime}\left\|T \wedge\left[\mathfrak{C}_{r}\right]\right\|_{\mathbb{A}_{r, N}}$. This, coupled with (9.5), implies that

$$
I \leq c(-\log r)^{-1} \log (-\log r) .
$$

Next, we turn to $(I I)$. Observe that every point $(z, w) \notin \mathbb{B}_{\kappa r} r^{1 / N}$ with $z_{N}(z, w)=$ $r$ satisfies the assumption of Proposition 10.3 for the sequence $M_{N}:=8^{N}$ as in (10.16). Therefore, we have for such a point that

$$
\left|z_{N}(z, w)-\left(z_{\infty}(z, w)+a_{N} w^{N}\right)\right| \leq 8^{-N}\left|a_{N}\right||w|^{N} .
$$

Consequently, we can argue as in the proof of Lemma 10.7 while replacing the ball $\mathbb{B}_{r^{1 / N}|\log r|^{3 / N}}$ with $\mathbb{B}_{\kappa r^{1 / N}}$. Thus we obtain the following weaker fact than Lemma 10.7 .

Claim. For $N$ large enough, there exist two numbers $\Gamma_{N}$ and $\Lambda_{N}$ such that by reducing $r_{N}$ if necessary, for every $0<r<r_{N}$ and $\alpha \in \mathbb{T}$, the following two properties hold:

(i) for every point $\xi_{1} \in\left(\mathfrak{C}_{0, N} \cap \widehat{L}_{\alpha}\right) \cap\left(\mathbb{A}_{r, N} \backslash \mathbb{B}_{\kappa r^{1 / N}}\right)$, there exist at least one point and at most $\Gamma_{N}$ points $\xi_{2} \in\left(\mathfrak{C}_{r, N} \cap \widehat{L}_{\alpha}\right) \cap\left(\mathbb{A}_{r, N} \backslash \mathbb{B}_{\kappa r^{1 / N}}\right)$ such that $\xi_{1}$ and $\xi_{2}$ are quasi-compatible in the sense of Definition 10.4 and $\operatorname{dist}_{C}\left(\xi_{1}, \xi_{2}\right) \leq \Lambda_{N}$

(ii) for every point $\xi_{1} \in\left(\mathfrak{C}_{r, N} \cap \widehat{L}_{\alpha}\right) \cap\left(\mathbb{A}_{r, N} \backslash \mathbb{B}_{\kappa r^{1 / N}}\right)$, there exist at least one point and at most $\Gamma_{N}$ points $\xi_{2} \in\left(\mathfrak{C}_{0, N} \cap \widehat{L}_{\alpha}\right) \cap\left(\mathbb{A}_{r, N} \backslash \mathbb{B}_{\kappa r^{1 / N}}\right)$ such that $\xi_{1}$ and $\xi_{2}$ are quasi-compatible and $\operatorname{dist}_{C}\left(\xi_{1}, \xi_{2}\right) \leq \Lambda_{N}$.

Sketchy proof of the claim. We only prove assertion (i) since assertion (ii) can be done similarly. Unlike the proof of Lemma 10.7, $s$ in this claim is a large positive number. Arguing as in the proof of (10.29), we may find a constant $c_{N}>1$ such that

$$
r<c_{N} \kappa^{-1}\left|a_{N} w_{1}^{N}\right|
$$


Now we choose $M$ large enough ( $M$ depending on $N$ ). In fact, instead of (10.30) $s$ in this claim is of the form

$$
s:=c^{\prime} \kappa^{-1} \quad \text { for } c^{\prime}=c_{N}^{\prime}>0 \text { a large constant independent of } r .
$$

As in the proof of Lemma 10.7 we want to estimate the number of roots $t$ of the following holomorphic function on $\mathbb{D}_{s}$ defined by (10.28):

$$
F(t):=R_{r}\left(e^{\lambda t} w_{1}\right)-\tilde{a}_{N} e^{\lambda N t} w_{1}^{N}+h\left(e^{\lambda t} w_{1}\right)+\tilde{a}_{N} e^{t} w_{1}^{N}-e^{t} h\left(w_{1}\right), \quad t \in \mathbb{D}_{s} .
$$

Consider again the function

$$
H(t):=-\tilde{a}_{N} e^{\lambda N t} w_{1}^{N}+\tilde{a}_{N} e^{t} w_{1}^{N}, \quad t \in \overline{\mathbb{D}}_{s} .
$$

Since $H(t)=0$ if and only if $t=\frac{2 i \pi k}{\lambda N-1}$ for $k \in \mathbb{Z}$, we may choose $s$ and $c^{\prime}$ large enough (depending only on $N$ and $\lambda$ ) such that

$$
|H(t)| \geq c^{\prime-1}\left|\tilde{a}_{N} w_{1}^{N}\right| \quad \text { for } \quad t \in \partial \mathbb{D}_{s}
$$

and that $H$ admits a finite number of roots, say $\Gamma_{N} \geq 1$ roots on $\mathbb{D}_{s}$. Using this and (10.18), (10.19), (10.23), (10.26), (10.29), (13.8) and (10.31), we can show that

$$
|F(t)-H(t)|<H(t) \quad \text { for } t \in \partial \mathbb{D}_{s} .
$$

Consequently, applying Rouché's theorem again to $F$ and $H$, the claim follows with $\Lambda_{N}:=s$.

Using the claim we may find a constant $c^{\prime \prime}=c_{N}^{\prime \prime}>1$ such that

$$
I I \leq c^{\prime \prime} \int_{\alpha \in \mathbb{T}}\left(\sum h_{\alpha}\left(\xi_{0, N, \alpha, k}\right)\right) d \nu(\alpha)=c^{\prime \prime}\left\|T \wedge\left[\mathfrak{C}_{0, N}\right]\right\|_{\mathbb{A}_{r, N} \backslash \mathbb{B}_{\kappa r} 1 / N},
$$

where the sum is taken over all $k \in \mathbb{N}$ such that $\xi_{0, N, \alpha, k} \in \mathbb{A}_{r, N} \backslash \mathbb{B}_{\kappa r^{1 / N}}$. The right hand side is bounded from above by $c^{\prime \prime}\left\|T \wedge\left[\mathfrak{C}_{0, N}\right]\right\|_{\mathbb{A}_{r, N}}$. This, coupled with (9.6), implies that

$$
I I \leq c(-\log r)^{-1} \log (-\log r)
$$

End of the proof of inequality (9.7) in Proposition 9.2. Putting (13.5), (13.6) and (13.9) altogether, (9.7) follows.

\section{Completion OF THE REDUCTions}

In the first part of this section we complete the proof of Proposition 8.6. More specifically, we will show that the geometric intersection $T \wedge\left[\mathfrak{C}_{0, N}\right]$ on $\mathbb{B}_{r^{1 / N}|\log r|^{-3 / N}}$ admits a coherent interpretation $K_{-\log r, N}^{*}$ satisfying the conclusion of this proposition. As a consequence, the second part is devoted to the proof of Proposition 8.4. 
14.1. End of the proof of Proposition 8.6. The proof is divided into 4 steps. Step 1: Construction of a coherent interpretation with mesh $N^{-2}$.

Let $d:=-a_{N}$ (see (8.7) and Lemma10.6 for $a_{N}$ ). We consider, for each $s>1$ and $N \in \mathbb{N} \backslash\{0\}$, the following domain in $\mathbb{R}$ :

$$
D_{s, N}^{*}:=\left\{t \in \mathbb{R}^{+}: t \geq N^{-1}(s-3 \log s)\right\},
$$

and the function $K_{s, N}^{*}: \mathbb{R} \rightarrow \mathbb{R}^{+}$given by

$$
K_{s, N}^{*}(y):=N^{2} \int_{D_{s, N}^{*}} \frac{V}{V^{2}+(y-U)^{2}} d t, \quad y \in \mathbb{R} .
$$

Here $U, V$ are functions of the variable $t$ which satisfy the following system of equations (see (5.6), (5.2) and (5.3)):

$$
U+i V=(u+i v)^{\gamma} \quad \text { and } \quad t=b u+a v \quad \text { and } \quad v=N t+\log |d| .
$$

For $\alpha \in \mathbb{T}$, set $D^{\alpha}:=D_{-\log r, N}^{*}$, and $K^{\alpha}:=K_{-\log r, N}^{*}$, and $\chi^{\alpha}(t)=u_{\alpha}(t)+i v_{\alpha}(t)$, $t \in D^{\alpha}$. Here $u_{\alpha}$ and $v_{\alpha}$ are affine functions in $t$ such that $u_{\alpha}\left(t_{N, \alpha, k}\right)=u_{N, \alpha, k}$ and $v_{\alpha}\left(t_{N, \alpha, k}\right)=v_{N, \alpha, k}$ for $k \in \mathbb{N}$ (see (10.3)-(10.4) in Lemma 10.2). We will show that $\left(K^{\alpha}\right)_{\alpha \in \mathbb{T}}$ is a coherent interpretation of the geometric intersection $T \wedge\left[\mathfrak{C}_{0, N}\right]$ on $\mathbb{B}_{r^{1 / N}|\log r|^{-3 / N} \text {. }}$

By Lemma 10.6 and using (5.1) we know that for every $\alpha \in \mathbb{T}$, each point $\xi=(z, w)=\psi_{\alpha}(u+i v) \in \mathfrak{C}_{N}^{d}=\left\{z=d w^{N}\right\} \cap \widehat{L}_{\alpha}$ corresponds to a unique point $\xi=(z, w)=\psi_{\alpha}(u+i v) \in \mathfrak{C}_{0, N} \cap \widehat{L}_{\alpha}$ such that $\xi$ and $\xi^{\prime}$ are compatible and $\operatorname{dist}_{C}\left(\xi_{N, \alpha, k}, \xi_{0, N, \alpha, k}\right) \lesssim N^{-1}$. Using Definition 10.4 we infer from the last inequality that

$$
\left|u-u^{\prime}\right| \lesssim N^{-1} \quad \text { and } \quad\left|v-v^{\prime}\right| \lesssim N^{-1} .
$$

On the other hand, by (10.5)-(10.6) in Lemma10.2, if $u:=u_{N, \alpha, k}, v:=v_{N, \alpha, k}$, $u^{\prime}:=u_{N, \alpha, k+1}, v^{\prime}:=v_{N, \alpha, k+1}$ for some $k \in \mathbb{N}$, we also get inequality (14.3).

Using (5.2) and (5.6), we set

$$
t=b u+a v, \quad t^{\prime}=b u^{\prime}+a v^{\prime}, \quad U+i V=(u+i v)^{\gamma}, \quad U^{\prime}+i V^{\prime}=\left(u^{\prime}+i v^{\prime}\right)^{\gamma} .
$$

This, combined with (14.3), yields that $t \approx t^{\prime}$ and $v \approx v^{\prime}$. Since $\xi \in \mathfrak{C}_{N}^{d}=\{z=$ $\left.d w^{N}\right\} \cap \widehat{L}_{\alpha} \cap \mathbb{B}_{r^{1 / N}|\log r|^{-3 / N}}$, it follows from (5.3) that

$$
t \geq-N^{-1} \log r+3 N^{-1} \log (-\log r) \quad \text { and } \quad v=N t+\log |d| .
$$

Note that the second estimate in (10.6) shows that the mesh of $\left(K^{\alpha}\right)_{\alpha \in \mathbb{T}}$ should be $N^{-2}$. Using the above estimates for $t, v$ and $t^{\prime}, v^{\prime}$ and applying Lemma 7.9, we can show that there is a constant $c>1$ independent of the above points $\xi, \xi^{\prime}$ such that

$$
c^{-1} \frac{V}{V^{2}+(y-U)^{2}} \leq \frac{V^{\prime}}{V^{\prime 2}+\left(y-U^{\prime}\right)^{2}} \leq c \frac{V}{V^{2}+(y-U)^{2}} \quad \text { for } \quad y \in \mathbb{R} .
$$


Therefore, by Definition 7.8, $\left(K^{\alpha}\right)_{\alpha \in \mathbb{T}}$ is a coherent interpretation of the geometric intersection $T \wedge\left[\mathfrak{C}_{0, N}\right]$ on $\mathbb{B}_{r^{1 / N}|\log r|^{-3 / N}}$ as desired. This completes Step 1.

Step 2: There are constant $c, \kappa>1$ independent of $N$ such that for $\kappa(1+|y|)^{1 / \gamma} \leq$ $s$, we have $K_{s, N}^{*}(y) \leq c s^{1-\gamma}$.

To start Step 2, let $c_{2}, c_{3}$ be the constants with $c_{3}>c_{2}>1$ given by Lemma 7.9. Set

$$
\kappa:=\max \left\{c_{2}, c_{3}\right\} .
$$

By Part 2) of Lemma 7.9 and by formula (14.2), we have that

$$
K_{s, N}^{*}(y) \approx \int_{t=s / N-3(\log s) / N}^{\infty} \frac{N^{2} t d t}{(N t)^{\gamma+1}} \approx s^{1-\gamma} .
$$

This completes the proof of Step 2.

Step 3: There are constant $c, \kappa>1$ independent of $N$ such that for $s \leq \kappa^{-1}(1+$ $|y|)^{1 / \gamma} \geq s$, we have $c^{-1} \leq \frac{K_{s, N}^{*}(y)}{N(1+|y|)^{1 / \gamma-1}} \leq c$.

Let $\kappa$ be given by Step 2. Applying Part 5) of Lemma 7.9 and using Step 2 above for $s=\kappa(1+|y|)^{1 / \gamma}$, we get that

$$
K_{s, N}^{*}(y) \approx(1+|y|)^{1 / \gamma-1}+\int_{t=s / N-3(\log s) / N}^{(1+|y|)^{1 / \gamma} / N} \frac{(1+|y|)^{1 / \gamma-1} N^{2} t d t}{t^{2}+(N t+\log |d|-\rho(y, t))^{2}}
$$

where $\rho(y, t)$ is defined as follows: there exists a solution $u:=u(y, t)$ of the following equation

$$
U=y, \quad U+i V=(u+i t)^{\gamma}
$$

satisfying $c_{2}^{-1}(1+|y|)^{1 / \gamma} \leq u, \rho(y, t) \leq c_{2}(1+|y|)^{1 / \gamma}$ with $\rho(y, t):=b u+a t$.

For every $k=1, \ldots, N$, let $t_{k}, u_{k} \in \mathbb{R}$ be such that

$$
\rho\left(y, t_{k}\right)=k t_{k} \quad \text { and } \quad \rho\left(y, t_{k}\right):=b u_{k}+a t_{k} .
$$

Observe that

$(N-k-1) t+\log |d| \leq N t+\log |d|-\rho(y, t) \leq(N-k) t+\log |d| \quad$ for $\quad t \in\left[t_{k+1}, t_{k}\right]$.

On the other hand, we deduce from (14.6) and (14.5) that

$$
t_{k}^{\gamma} \operatorname{Re}\left(\left(b^{-1}(k-a)+i\right)^{\gamma}\right)=\operatorname{Re}\left(\left(b^{-1}(k-a) t_{k}+i t_{k}\right)^{\gamma}\right)=\operatorname{Re}\left(\left(u_{k}+i t_{k}\right)^{\gamma}\right)=y .
$$

This, combined with the estimate

$$
\operatorname{Re}\left(\left(b^{-1}(k-a)+i\right)^{\gamma}\right) \approx k^{\gamma} \text { for large } k,
$$

implies the following estimates

$$
t_{k} \approx k^{-1}|y|^{1 / \gamma},
$$


and

$$
\begin{aligned}
t_{k-1}-t_{k} & \approx t_{k}^{\gamma+1}\left(t_{k}^{\gamma}-t_{k-1}^{\gamma}\right) \\
& \approx k^{-(\gamma+1)}|y|^{1+1 / \gamma} \frac{\operatorname{Re}\left(\left(b^{-1}(k-a)+i\right)^{\gamma}\right)-\operatorname{Re}\left(\left(b^{-1}(k-a)+i\right)^{\gamma}\right)}{|y|} \\
& \approx k^{-1}|y|^{1 / \gamma}\left(\operatorname{Re}\left(\left(b^{-1}\left(1-a k^{-1}\right)+i k^{-1}\right)^{\gamma}\right)-\operatorname{Re}\left(\left(b^{-1}\left(1-a k^{-1}\right)+i k^{-1}\right)^{\gamma}\right)\right) \\
& \approx k^{-2}|y|^{1 / \gamma} .
\end{aligned}
$$

Inserting these inequalities into (14.7) and hence (14.4), we get that

$$
\begin{aligned}
K_{s, N}^{*}(y) & \approx(1+|y|)^{1 / \gamma-1}+N^{2} \sum_{k=1}^{N} \frac{\left(t_{k-1}-t_{k}\right)(1+|y|)^{1 / \gamma-1} t_{k}}{t_{k}^{2}+\left(N t_{k}+\log |d|-\rho\left(y, t_{k}\right)\right)^{2}} \\
& \approx(1+|y|)^{1 / \gamma-1}+N^{2}(1+|y|)^{1 / \gamma-1} \sum_{k=1}^{N} \frac{\left(t_{k-1}-t_{k}\right)}{t_{k}(N-k)^{2}} \\
& \approx(1+|y|)^{1 / \gamma-1}+N^{2}(1+|y|)^{1 / \gamma-1} \sum_{k=1}^{N-1} \frac{k^{-2}|y|^{1 / \gamma}}{k^{-1}|y|^{1 / \gamma}(N-k)^{2}} \\
& \approx(1+|y|)^{1 / \gamma-1}\left(1+N^{2} \sum_{k=1}^{N-1} \frac{1}{k(N-k)^{2}}\right) \\
& \approx N(1+|y|)^{1 / \gamma-1},
\end{aligned}
$$

where in the second $\approx$ we use that $-N \log 2 \leq \log |d| \leq N \log 2$, which follows, in turn, from the first two inequalities in (10.9).

Step 4: There are a constant $\kappa>1$ independent of $N$ and a constant $c_{N}>1$ such that for $\kappa^{-1} s \leq(1+|y|)^{1 / \gamma} \leq \kappa s$, we have $c_{N}^{-1} \leq \frac{K_{s, N}^{*}(y)}{(1+|y|)^{1 / \gamma-1}} \leq c_{N}$.

We use Lemma7.9 in order to estimate $K_{s, N}^{*}(y)$. Since this step is much easier than Step 2 and Step 3, we leave it to the interested reader.

Putting Step 1, 2, 3 and 4 altogether, the proof of Proposition 8.6 is thereby completed.

14.2. End of the proof of Proposition 8.4. We apply what has been done in this section to $\mathbb{B}_{r^{1 / N}}$ instead of $\mathbb{B}_{r^{1 / N}|\log r|^{-3 / N}}$. Consequently, we obtain quite similar estimates as in Step 2, 3, 4 above. This, combined with Lemma 6.1 for $r^{1 / N}$ instead of $r$, yields a constant $c=c_{N}>0$ such that

$$
\left\|T \wedge\left[\mathfrak{C}_{0, N}\right]\right\|_{\mathbb{B}_{r^{1 / N}}} \leq c G\left(x_{0}, r^{1 / N}\right) \text { for } 0<r<1 / 2 .
$$

Replacing $r^{1 / N}$ by $r$, the result follows.

\section{REFERENCES}

[1] Berndtsson, Bo; Sibony, Nessim. The $\bar{\partial}$-equation on a positive current. Invent. Math. 147 (2002), no. 2, 371-428. 
[2] Brunella, Marco. Inexistence of invariant measures for generic rational differential equations in the complex domain, Bol. Soc. Mat. Mexicana (3), 12 (2006), no. 1, 4349.

[3] Candel, Alberto. Uniformization of surface laminations, Ann. Sci. École Norm. Sup. (4), 26 (1993), no. 4, 489-516.

[4] —. The harmonic measures of Lucy Garnett, Adv. Math., 176 (2003), no. 2, 187-247.

[5] Candel, Alberto; Conlon, Lawrence. Foliations. I. Graduate Studies in Mathematics, 23. American Mathematical Society, Providence, RI, 2000. xiv+402 pp.

[6] — . Foliations. II. Graduate Studies in Mathematics, 60. American Mathematical Society, Providence, $R I$, 2003. xiv +545 pp.

[7] Chavel, Isaac. Eigenvalues in Riemannian geometry. Including a chapter by Burton Randol. With an appendix by Jozef Dodziuk. Pure and Applied Mathematics, 115. Academic Press, Inc., Orlando, FL, 1984. xiv+362 pp.

[8] Deroin, Bertrand. Hypersurfaces Levi-plates immergées dans les surfaces complexes de courbure positive. (French) [Immersed Levi-flat hypersurfaces in complex surfaces of positive curvature]. Ann. Sci. École Norm. Sup. (4) 38 (2005), no. 1, 57-75.

[9] — - Brownian motion on foliated complex surfaces, Lyapunov exponents and applications. Geometry and Foliations, 2013, Komaba, Tokyo, Japan.

[10] Dinh, T.-C.; Nguyên, V.-A.; Sibony, N. Heat equation and ergodic theorems for Riemann surface laminations. Math. Ann. 354, (2012), no. 1, 331-376.

[11] - Entropy for hyperbolic Riemann surface laminations I. Frontiers in Complex Dynamics: a volume in honor of John Milnor's 80th birthday, (A. Bonifant, M. Lyubich, S. Sutherland, editors), 569-592, (2014), Princeton University Press.

[12] - Entropy for hyperbolic Riemann surface laminations II. Frontiers in Complex Dynamics: a volume in honor of John Milnor's 80th birthday, (A. Bonifant, M. Lyubich, S. Sutherland, editors), 593-622, (2014), Princeton University Press.

[13] Dinh, T.-C.; Sibony, N. Unique ergodicity for foliations in $\mathbb{P}^{2}$ with an invariant curve. Invent. math., to appear. DOI 10.1007/s00222-017-0744-2

[14] Fornæss, John Erik; Sibony, Nessim. Harmonic currents of finite energy and laminations. Geom. Funct. Anal., 15 (2005), no. 5, 962-1003.

[15] —. Riemann surface laminations with singularities. J. Geom. Anal., 18 (2008), no. 2, 400-442.

[16] —. Unique ergodicity of harmonic currents on singular foliations of $\mathbb{P}^{2}$. Geom. Funct. Anal., 19 (2010), no. 5, 1334-1377.

[17] Garnett, Lucy. Foliations, the ergodic theorem and Brownian motion. J. Funct. Anal. 51 (1983), no. 3, 285-311.

[18] Ghys, Étienne. Laminations par surfaces de Riemann. (French) [Laminations by Riemann surfaces] Dynamique et géométrie complexes (Lyon, 1997), ix, xi, 49-95, Panor. Synthèses, 8, Soc. Math. France, Paris, 1999.

[19] Glutsyuk, A.A. Hyperbolicity of the leaves of a generic one-dimensional holomorphic foliation on a nonsingular projective algebraic variety. (Russian) Tr. Mat. Inst. Steklova, 213 (1997), Differ. Uravn. s Veshchestv. i Kompleks. Vrem., 90-111; translation in Proc. Steklov Inst. Math. 1996, no. 2, 213, 83-103.

[20] Hurder, Steven. Classifying foliations. Foliations, geometry, and topology, 1-65, Contemp. Math., 498, Amer. Math. Soc., Providence, RI, 2009.

[21] Hussenot, Nicolas. On the dynamics of Riccati foliations with non parabolic monodromy representations. math.DS, math.CV, arXiv:1604.06689v2, 28 pages.

[22] Lins Neto A. Simultaneous uniformization for the leaves of projective foliations by curves. Bol. Soc. Brasil. Mat. (N.S.), 25 (1994), no. 2, 181-206. 
[23] - Uniformization and the Poincaré metric on the leaves of a foliation by curves. Bol. Soc. Brasil. Mat. (N.S.), 31 (2000), no. 3, 351-366.

[24] Lins Neto, A.; Soares M. G. Algebraic solutions of one-dimensional foliations. J. Differential Geom., 43 (1996), no. 3, 652-673.

[25] Nguyên, Viêt-Anh. Oseledec multiplicative ergodic theorem for laminations. Mem. Amer. Math. Soc. 246 (2017), no. 1164, ix+174 pp.

[26] —. Geometric characterization of Lyapunov exponents for laminations. J. Geom. Anal., to appear. DOI : $10.1007 / \mathrm{s} 12220-017-9919-8$

[27] — Directed harmonic currents near hyperbolic singularities. Ergodic Theory Dynam. Systems, to appear. DOI: https://doi.org/10.1017/etds.2017.2

[28] Skoda, Henri. Prolongement des courants, positifs, fermés de masse finie. (French) [Extension of closed, positive currents of finite mass] Invent. Math. 66 (1982), no. 3, 361376.

[29] Sullivan, Dennis. Cycles for the dynamical study of foliated manifolds and complex manifolds. Invent. Math. 36 (1976), 225-255.

[30] Walczak, Pawel. Dynamics of foliations, groups and pseudogroups. Instytut Matematyczny Polskiej Akademii Nauk. Monografie Matematyczne (New Series) [Mathematics Institute of the Polish Academy of Sciences. Mathematical Monographs (New Series)], 64. Birkhäuser Verlag, Basel, 2004. xii+225 pp.

Viêt-Anh Nguyên, Université de Lille 1, Laboratoire de mathématiques Paul Painlevé, CNRS U.M.R. 8524, 59655 Villeneuve d'Ascq Cedex, France.

Viet-Anh.Nguyen@math.univ-lille1.fr, http://math.univ-lille1.fr/ vnguyen 